\title{
A Machine-Checked Model for a Java-Like Language, Virtual Machine, and Compiler
}

\author{
GERWIN KLEIN \\ National ICT Australia \\ and \\ TOBIAS NIPKOW \\ Technische Universität München
}

\begin{abstract}
We introduce Jinja, a Java-like programming language with a formal semantics designed to exhibit core features of the Java language architecture. Jinja is a compromise between the realism of the language and the tractability and clarity of its formal semantics. The following aspects are formalised: a big and a small step operational semantics for Jinja and a proof of their equivalence, a type system and a definite initialisation analysis, a type safety proof of the small step semantics, a virtual machine (JVM), its operational semantics and its type system, a type safety proof for the JVM; a bytecode verifier, that is, a data flow analyser for the JVM, a correctness proof of the bytecode verifier with respect to the type system, and a compiler and a proof that it preserves semantics and well-typedness. The emphasis of this work is not on particular language features but on providing a unified model of the source language, the virtual machine, and the compiler. The whole development has been carried out in the theorem prover Isabelle/HOL.

Categories and Subject Descriptors: D.3.1 [Programming Languages]: Formal Definitions and Theory-Semantics; D.3.4 [Programming Languages]: Processors-Compilers; F.3.3 [Logics and Meanings of Programs]: Studies of Program Constructs-Object-oriented constructs
\end{abstract}

General Terms: Languages, Verification

Additional Key Words and Phrases: Java, operational semantics, theorem proving

\section{INTRODUCTION}

There is a large body of literature on formal models of Java-like languages, the virtual machine and bytecode verification, and compilation. However, each of these models is designed to treat certain features of Java in depth while

This work was done while T. Nipkow was on sabbatical at NICTA where he was supported by the NICTA Formal Methods program and the DFG.

Authors' addresses: G. Klein, National ICT Australia, University of New South Wales, Sydney NSW 2052, Australia; T. Nipkow, Institut für Informatik, Technische Universität München, Boltzmannstraße 3, 85748 Garching, Germany.

Permission to make digital or hard copies of part or all of this work for personal or classroom use is granted without fee provided that copies are not made or distributed for profit or direct commercial advantage and that copies show this notice on the first page or initial screen of a display along with the full citation. Copyrights for components of this work owned by others than ACM must be honored. Abstracting with credit is permitted. To copy otherwise, to republish, to post on servers, to redistribute to lists, or to use any component of this work in other works requires prior specific permission and/or a fee. Permissions may be requested from Publications Dept., ACM, Inc., 1515 Broadway, New York, NY 10036 USA, fax: +1 (212) 869-0481, or permissions@acm.org.

(C) 2006 ACM 0164-0925/06/0700-0619 \$5.00 
ignoring other features completely (see the following for one exception). The main result of our work is the first unified model of a Java-like source language, virtual machine, and compiler, with the following salient features: It is based on operational semantics, is executable, fits into the confines of a journal article, and all proofs are machine-checked (in Isabelle/HOL [Nipkow et al. 2002]). This is considerably more than the sum of its parts. A tractable formal model is the result of a careful balance of features on each level and the interaction between the different levels (here, the source and target language). That is, integrating different formal models is just as much of a challenge as designing the individual models.

In addition to the unified model, there are also a number of specific advances of the state-of-the art: a big and small step semantics together with an equivalence proof (previously, only one or the other had been used), a formalisation of the "definite assignment" analysis of local variables, and a compiler correctness proof covering exceptions. We discuss these issues in detail in the respective sections.

It must be emphasised that our work is complementary to the detailed analysis of particular language features. In the end, a synthesis of our unified (but in many places, simplified) model and more detailed (but specialised) models should emerge. And because of the unavoidable size of the resulting model, we believe that some machine-checked analysis, ideally with a theorem prover, is necessary to guarantee the overall consistency of the model-in particular, when faced with changes. With each change to a model of that size, a purely textual definition becomes less and less reliable; in the absence of mechanical consistency checks, changes in one part of the model will sooner or later have unexpected side effects in other parts. This is where formal verification and the ability to rerun proofs really pays off. But even without formal proofs, mere type checking is already tremendously beneficial. Hence, this article should also be viewed as an attempt to combine the rigour of a formal machine-checked metalanguage with standard (largely mathematical) notation. There will be more coverage of this issue in Section 1.2.

The article is subdivided into four parts: the source language (Section 2), the virtual machine (Section 3), the bytecode verifier (Section 4), and the compiler (Section 5).

We discuss related work separately at the end of each section because most articles deal with a specific language layer. The exception is a book by Stärk et al. [2001] which treats almost all of Java and the virtual machine. This is a very impressive piece of work, but quite different from ours; it is based on abstract state machines rather than on standard operational semantics, and the proofs are not machine-checked. It should be noted that the literature on formal models of Java and the JVM is already so large that it gave rise to three survey-like publications [Alves-Foss 1999; Hartel and Moreau 2001; Nipkow 2003a]. Hence, our discussion of related work is necessarily restricted to those articles with a very direct connection to ours.

As a final word of warning, we must emphasize that the core of the article is intentionally detailed and technical. Its very aim is to demonstrate the stateof-the-art in machine-checked language definitions. 


\subsection{Basic Notation-The Metalanguage}

Our metalanguage HOL conforms largely to everyday mathematical notation. This section introduces further nonstandard notation and, in particular, a few basic data types with their primitive operations.

Types. The basic types of truth values, natural numbers, and integers are called bool, nat, and int, respectively. The space of total functions is denoted by $\Rightarrow$. Type variables are written ' $a$, ' $b$, etc. The notation $t:: \tau$ means that the HOL term $t$ has HOL type $\tau$.

Pairs. Pairs come with the two projection functions $f s t::{ }^{\prime} a \times{ }^{\prime} b \Rightarrow{ }^{\prime} a$ and snd $::{ }^{\prime} a \times{ }^{\prime} b \Rightarrow{ }^{\prime} b$. We identify tuples with pairs nested to the right: $(a, b, c)$ is identical to $(a,(b, c))$ and ' $a \times{ }^{\prime} b \times{ }^{\prime} c$ is identical to ${ }^{\prime} a \times\left({ }^{\prime} b \times{ }^{\prime} c\right)$.

Sets. Sets (type 'a set) follow the usual mathematical convention. Intervals are written as follows: $\{m . .<n\}$ means $\{i \mid m \leq i<n\}$ and $\{m . . n\}$ means $\{i \mid m \leq$ $i \leq n\}$. If $m$ is 0 , it can be dropped.

Lists. Lists (type 'a list) come with the empty list [], the infix constructor ., the infix @ that appends two lists, and the conversion function set from lists to sets. Variable names ending in "s" usually stand for lists, $|x s|$ is the length of $x s$, and $x s_{[n]}$, where $n:: n a t$, is the $n$ th-element of $x s$ (starting with 0 ). The notation $[i . .<j]$ with $i::$ nat and $j:: n a t$ stands for the list $[i, \ldots, j-1]$. The formula distinct $x s$ means that the elements of $x s$ are all distinct. The standard functions map and filter are also available.

\section{datatype 'a option $=$ None $\mid$ Some 'a}

adjoins a new element None to a type ' $a$. For succinctness, we write $\lfloor a\rfloor$ instead of Some $a$. The underspecified inverse the of Some satisfies the $\lfloor x\rfloor=x$.

Function update is written $f(x:=y)$, where $f::{ }^{\prime} a \Rightarrow{ }^{\prime} b, x::{ }^{\prime} a$ and $y:: ' b$.

Partial functions are modelled as functions of type ' $a \Rightarrow{ }^{\prime} b$ option, where None represents undefinedness and $f x=\lfloor y\rfloor$ means $x$ is mapped to $y$. We define dom $m \equiv\{a \mid m a \neq$ None $\}$. Instead of ' $a \Rightarrow{ }^{\prime} b$ option, we write ' $a \rightarrow{ }^{\prime} b$, call such functions maps, and abbreviate $f(x:=\lfloor y\rfloor)$ to $f(x \mapsto y)$. The latter notation extends to lists: $f\left(\left[x_{1}, \ldots, x_{m}\right][\mapsto]\left[y_{1}, \ldots, y_{n}\right]\right)$ means $f\left(x_{1} \mapsto y_{1}\right) \ldots\left(x_{i} \mapsto y_{i}\right)$, where $i$ is the minimum of $m$ and $n$. The notation works for arbitrary list expressions on both sides of $[\mapsto]$, not just enumerations. Multiple updates like $f(x \mapsto y)(x s[\mapsto] y s)$ can be written as $f(x \mapsto y, x s[\mapsto] y s)$. The map $\lambda x$. None is written empty, and empty (...), where ... are updates, abbreviates to [...]. For example, empty $(x \mapsto y$, $x s[\mapsto] y s)$ becomes $[x \mapsto y, x s[\mapsto] y s]$.

Overwriting map $m_{1}$ with $m_{2}$ is written $m_{1}++m_{2}$ and means $\lambda x$. case $m_{2} x$ of None $\Rightarrow m_{1} x \mid\lfloor y\rfloor \Rightarrow\lfloor y\rfloor$.

Function map-of turns an association list, that is, a list of pairs, into a map:

map-of []$=$ empty

map-of $(p \cdot p s)=$ map-of $p s(f s t p \mapsto s n d p)$

Note that $\llbracket A_{1} ; \ldots ; A_{n} \rrbracket \Longrightarrow A$ abbreviates $A_{1} \Longrightarrow\left(\ldots \Longrightarrow\left(A_{n} \Longrightarrow A\right) \ldots\right)$. In lemmas we often write "If $A_{1}$ and $\ldots$ and $A_{n}$ then $A$," instead. Displayed implications are frequently printed as inference rules: 


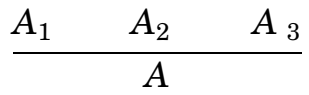

\subsection{Presentation Issues}

How are the formulae you see related to the formal Isabelle text? Our motto is

What you see is what we proved!

In many articles this is not the case. For example, the main definition by Nipkow [1991] and the main theorem, Theorem 1, by Flatt et al. [1999] are blatantly wrong (the latter misses the case that $e$ might diverge). In order to avoid this problem, Isabelle theories can be augmented with $\mathrm{HT}_{\mathrm{E}} \mathrm{X}$ text which may contain references to Isabelle theorems (by name-see Chapter 4 of the book by Nipkow et al. [2002]). When this $\mathrm{LT}_{\mathrm{E}} \mathrm{X}$ text is processed by Isabelle, it expands these references into the $\mathrm{HT}_{\mathrm{E}} \mathrm{X}$ text for the proposition of the theorem. Using this mechanism, the text for most of the definitions and theorems in this article is automatically generated. We emphasize that the $\mathrm{IT}_{\mathrm{EX}}$ presentation is quite close to the original Isabelle theories viewed with the help of the proof general interface [Aspinall 2000] because the latter supports mathematical symbols. Only a few niceties like inference rules are $\mathrm{H}_{\mathrm{E}} \mathrm{X}$-specific.

It is conceivable to go one step further and even generate proof text automatically from the theories. However, although most of our proofs are in a quasireadable form of structured stylized mathematics [Wenzel 2002; Nipkow $2003 \mathrm{~b}]$, it appears beyond the state-of-the-art to turn these into concise journalstyle proofs automatically.

\section{JINJA}

Our dialect of Java is called Jinja (because Jinja is not Java). Although Jinja is a typed language, we begin its description with the operational semantics which is independent of the type system. First we introduce a big step or evaluation semantics (Section 2.2), then a small step or reduction semantics (Section 2.3). The big step semantics will be used in the compiler proof, the small step semantics in the type safety proof. Both semantics are defensive: rules only apply if everything "fits together." For example, variable lookup requires that the variable has been initialised. This is a prerequisite for the type safety proof, which shows that reduction of well-typed expressions does not get stuck. In Section 2.4 certain minimal well-formedness conditions for programs are defined. They imply (Section 2.5) that the two semantics are equivalent for terminating executions. Then we introduce a type system (Section 2.6) and "definite assignment" analysis (Section 2.7) for expressions and complete the set of wellformedness conditions for Jinja programs (Section 2.8). Finally, we show type safety (Section 2.9).

\subsection{Abstract Syntax}

2.1.1 Names. In the sequel we use the following (HOL) variable conventions: $V$ is a (Jinja) variable name, $F$ a field name, $M$ a method name, $C$ a class name, $e$ an expression, $v$ a value, $T$ a type, and $P$ a program. 
For readability only, we have introduced three (HOL) types for Jinja identifiers: cname (class names), vname (variable names), and mname (method names). All three are merely synonyms for type string.

\subsubsection{Values and Expressions. A Jinja value can be}

—a Boolean, Bool $b$, where $b$ :: bool, or

—an integer, Intg $i$, where $i::$ int, or

— a reference, Addr $a$, where $a:: a d d r$, or

- the null reference, Null, or

- the dummy value, Unit.

Jinja is an imperative but expression-based language where statements are expressions that evaluate to Unit. To make things executable, type $a d d r$ is actually a synonym for type nat (as opposed to leaving it unspecified).

The following expressions (of HOL type expr) are supported by Jinja:

-creation of new object: new $C$

- casting: Cast $C e$

-literal value: Val $v$

-binary operation: $e_{1} \ll b o p \gg e_{2}$ (where bop is one of + or $=$ )

- variable access Var $V$ and variable assignment $V:=e$

- field access $e . F\{D\}$ and field assignment $e_{1} . F\{D\}:=e_{2}$

(where $D$ is the class where $F$ is declared)

- method call: e.M(es)

—block with locally declared variable: $\{V: T ; e\}$

- sequential composition: $e_{1} ; e_{2}$

-conditional: if (e) $e_{1}$ else $e_{2}$ (do not confuse with HOL's if $b$ then $x$ else $y$ )

-while loop: while $(e) e^{\prime}$

- exception throwing throw $e$ and catching try $e_{1} \operatorname{catch}(C V) e_{2}$

The constructors Val and Var are needed in our metalanguage to disambiguate the syntax. There is no return statement because everything is an expression and returns a value.

Note that the annotation $\{D\}$ in field access and assignment is not part of the input language but is something that a preprocessor, for example, the type checking phase of a compiler, must add. We come back to this point in Section 2.6.

To ease notation we introduce some abbreviations:

$$
\begin{array}{lllll}
\text { true } & \equiv \operatorname{Val}(\text { Bool True }) & \text { false } & \equiv \text { Val Bool False }) \\
\text { addra } & \equiv \operatorname{Val}(\text { Addra) } & \text { null } & \equiv \text { Val Null } \\
\text { unit } & \equiv \operatorname{Val} \text { Unit } & & &
\end{array}
$$

Jinja supports only the two binary operators $=$ and + to keep things simple. Their evaluation is defined via a function binop that takes an operator and two 


$$
\begin{aligned}
\text { types } & =\text { 'm prog cdecl list } \\
\text { 'm cdecl } & =\text { cname } \times \text { 'm class } \\
\text { 'm class } & =\text { cname } \times \text { fdecl list } \times{ }^{\prime} m \text { mdecl list } \\
\text { fdecl } & =\text { vname } \times \text { ty } \\
\text { 'm mdecl } & =\text { mname } \times \text { ty list } \times \text { ty } \times{ }^{\prime} m \\
J-m b & =\text { vname list } \times \text { expr } \\
J-p r o g & =J-m b \text { prog }
\end{aligned}
$$

Fig. 1. Abstract program syntax.

values and returns an optional value (to deal with type mismatches):

$$
\begin{array}{ll}
\text { binop }\left(=, v_{1}, v_{2}\right) & =\left\lfloor\operatorname{Bool}\left(v_{1}=v_{2}\right)\right\rfloor \\
\text { binop }\left(+, \text { Intg } i_{1}, \text { Intg } i_{2}\right) & =\left\lfloor\operatorname{Intg}\left(i_{1}+i_{2}\right)\right\rfloor \\
\text { binop }\left(\text { bop }, v_{1}, v_{2}\right) & =\text { None }
\end{array}
$$

Addition only yields a value if both arguments are integers. We could also insist on similar compatibility checks for the equality test, but this leads to excessive case distinctions that we want to avoid for reasons of presentation.

2.1.3 Programs. The abstract syntax of programs is given by the type definitions in Figure 1, where ty is the HOL type of Jinja types. A program is a list of class declarations. A class declaration consists of the name of the class and the class itself. A class consists of the name of its direct superclass, a list of field declarations, and a list of method declarations. A field declaration is a pair consisting of a field name and its type. A method declaration consists of the method name, the parameter types, the result type, and the method body.

The only unusual thing here is the parameterisation of these types by ' $m$, the type of method body. The reason is that we want to use the program structure not just for Jinja but also for virtual machine programs. All we need to do is slot in the right kind of method body: a Jinja method body $J-m b$ is a pair of formal parameter names and the expression, and a Jinja program $J$-prog is a program with Jinja method bodies. Note that parameter names cannot be part of the generic syntax because there are no parameter names on the virtual machine level.

This generic program syntax is an important abstraction that will also be useful during compilation. More concrete representations, for example, concrete source language syntax or Java's class file format, are orthogonal to our work and can be added separately.

2.1.4 Extracting Declaration Information. Most of the time the exact representation of programs is irrelevant because we work in terms of a few functions and predicates for analysing and accessing the declarations in a program:

- class $P C$ is the class associated with $C$ in $P$.

-is-class $P C$ means class $C$ is defined in $P$.

$-P \vdash D \preceq^{*} C$ means $D$ is a subclass of $C$. The relation is transitive and reflexive.

$-P \vdash C$ sees $M: T s \rightarrow T=m b$ in $D$ means that in $P$, scanning the class hierarchy upwards starting from $C$, a method $M$ is visible in class $D$ (taking overriding 
into account). Its argument types are $T s$ (a type list), its result type is $T$, and its body is $m b$. If $P$ is a Jinja program, $m b$ is a pair (pns, $e$ ) of formal parameter list pns and an expression $e$.

$-P \vdash C$ sees $F: T$ in $D$ means that in $P$ from class $C$, a field $F$ of type $T$ is visible in class $D$.

$-P \vdash C$ has $F: T$ in $D$ means that in $P$, a (not necessarily proper) superclass $D$ of $C$ has a field $F$ of type $T$.

Before we show the definition of these predicates, we give an example (in an imaginary concrete syntax) that should clarify the concepts:

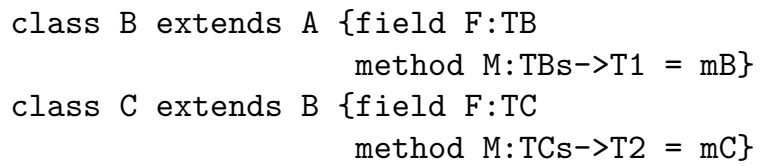

We have $P \vdash C$ sees $F$ :TC in $C$, but not $P \vdash C$ sees $F: T B$ in $B$ because the declaration in $C$ hides the one in $B$. In contrast, we have both $P \vdash C$ has $F: T C$ in $C$ and $P \vdash C$ has $F$ :TB in $B$ because has is independent of visibility.

Analogously, we have $P \vdash B$ sees $M: T B s \rightarrow T_{1}=m B$ in $B$ and $P \vdash C$ sees $M$ : $T C s \rightarrow T_{2}=m C$ in $C$, but not $P \vdash C$ sees $M: T B s \rightarrow T_{1}=m B$ in $B$. The second declaration of $M$ overrides the first, no matter what type of $M$ is in the two declarations.

This is method overriding in its purest form, but differs from Java where methods can also be overloaded, which means that multiple declarations of $M$ can be visible simultaneously, provided they are distinguished by their argument types. We have formalised overloading elsewhere [Oheimb and Nipkow 1999] but have not included it in Jinja. It complicates matters without adding a significant new aspect; we have to annotate method calls with the static type of the actual parameter list, just as in field access. This mixture of static type (for the parameter list) and dynamic type (of the object at runtime) can make programs with overloading hard to understand.

We will now show how the aforementioned functions are defined. The first two are trivial: class $\equiv$ map-of and is-class $P C \equiv$ class $P C \neq N o n e$.

For the remaining functions, it is important to note that we do not differentiate between user-defined classes and system classes. That is, any proper program will need to contain a class Object. Since any class has a superclass entry, so has Object. Therefore, class Object is treated specially: Traversal of the class hierarchy stops there and the dummy superclass entry is ignored.

The subclass relation $\swarrow^{*}$ is defined as the reflexive transitive closure of the direct subclass relation $\prec^{1}$ defined by

$$
\frac{\text { class } P C=\lfloor(D, f s, m s)\rfloor \quad C \neq \text { Object }}{P \vdash C \prec^{1} D}
$$

The information about fields in a class hierarchy is collected by a predicate $P \vdash C$ has-fields FDTs, where FDTs :: ((vname $\times$ cname $) \times$ ty) list. That is, each tuple $((F, D), T)$ in $F D T s$ represents a field declaration $F: T$ in a superclass $D$ of $C$, and the list follows the class hierarchy, that is, the fields of $C$ itself come 


$$
\begin{aligned}
\text { types } & =\text { heap } \times \text { locals } \quad \text { obj }=\text { cname } \times \text { fields } \\
\text { locals } & =\text { vname } \rightarrow \text { val } \text { fields }=\text { vname } \times \text { cname } \rightarrow \text { val } \\
\text { heap } & =a d d r \rightarrow \text { obj }
\end{aligned}
$$

Fig. 2. The types of Jinja program states.

first in the list. The predicate is defined inductively:

$$
\begin{gathered}
\frac{\text { class } P C=\lfloor(D, f s, m s)\rfloor \quad C \neq \text { Object } P \vdash D \text { has-fields F DTs }}{P \vdash C \text { has-fields map }(\lambda(F, T) .((F, C), T)) f s @ F D T s} \\
\frac{\text { class } P \text { Object }=\lfloor(D, f s, m s)\rfloor}{P \vdash \text { Object has-fields map }(\lambda(F, T) .((F, \text { Object }), T)) f s}
\end{gathered}
$$

At the moment, we do not rule out class Object having fields. In our preceeding example, assuming A is in fact Object, and assuming Object does not have fields, we obtain $P \vdash C$ has-fields [( $(F, C), T C),((F, B), T B)]$.

From the has-fields relation we can define has and sees directly:

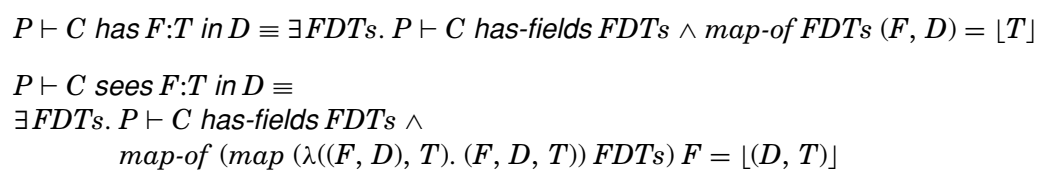

The relation sees for methods can be defined analogously via a relation that traverses the class hierarchy and collects all method declaration information. We omit the details.

\subsection{Big Step Semantics}

2.2.1 State. The type of states during expression evaluation is defined in Figure 2. A state is a pair of a heap and a store (locals). A store is a map from variable names to values. A heap is a map from addresses to objects. An object is a pair consisting of a class name and a field table, and a field table is a map from pairs $(F, D)$ (where $D$ is the class where $F$ is declared) to values. It is essential to include $D$ because an object may have multiple fields of the same name, all of them visible.

The naming convention is that $h$ is a heap, $l$ is a store (the local variables), and $s$ is a state. The projection functions $h p$ and $l c l$ are synonyms for $f s t$ and snd.

When a new object is allocated on the heap, its fields are initialised with the default value determined by their type:

init-fields $::(($ vname $\times$ cname $) \times$ ty $)$ list $\Rightarrow$ fields

init-fields $\equiv$ map-of $\circ$ map $(\lambda(F, T) .(F$, default-val $T))$

The definition of the default value is irrelevant to our purposes. It suffices to know that the default value is of the right type and that for references (types Class and NT), it is Null.

2.2.2 Evaluation. The evaluation judgement is of the form $P \vdash\langle e, s\rangle \Rightarrow$ $\left\langle e^{\prime}, s\right\rangle$, where $e$ and $s$ are the initial expression and state, respectively, and $e^{\prime}$ and $s^{\prime}$ the final expression and state, respectively. We then say that e evaluates 
to $e^{\prime}$. The rules will be such that final expressions are always values (Val) or exceptions (throw), that is, final expressions are completely evaluated. We will discuss the evaluation rules in an incremental fashion; first, normal evaluation only, and then exceptional behaviour afterwards.

2.2.3 Normal Evaluation. Normal evaluation means that we are defining an exception-free language. In particular, all final expressions will be values. The complete set of rules is shown in Figure 3 and we discuss them in turn.

Expression new $C$ first allocates a new address: function new-Addr (we omit its definition) returns a "new" address, that is, new-Addr $h=\lfloor a\rfloor$ implies $h a=$ None. Then predicate has-fields (Section 2.1.4) computes the list of all field declarations in and above $C$, and init-fields (Section 2.2.1) creates the default field table.

There are two rules for Cast $C e$ : If $e$ evaluates to the address of an object of a subclass of $C$ or to null, the cast succeeds, in the latter case because the null reference is in every class.

The rules for Val, Var, and assignment are self-explanatory.

Field access e.F $\{D\}$ evaluates $e$ to an address, looks up the object at the address, indexes its field table with $(F, D)$, and evaluates to the value found in the field table. Note that field lookup follows a static binding discipline: The dynamic class $C$ is ignored and the annotation $D$ is used instead. Later on, well-typedness will require $D$ to be the first class where $F$ is declared when we start looking from the static class of $e$ up the class hierarchy.

Field assignment $e_{1} \cdot F\{D\}:=e_{2}$ evaluates $e_{1}$ to an address and $e_{2}$ to a value, updates the object at the address with the value (using the index $(F, D)$ ), and evaluates to unit (just like assignment to local variables).

Why does assignment not evaluate to the value of the rhs, like in Java? Because then, the conditional if $(e) V_{1}:=e_{1}$ else $V_{2}:=e_{2}$ would not evaluate to unit but to the value of $e_{1}$ or $e_{2}$. Thus, the type system (to be definend later) would require $e_{1}$ and $e_{2}$ to have compatible types, which in many cases they wouldn't, thus forcing the programmer to write something like if $(e)\left(V_{1}:=e_{1}\right.$; unit) else $\left(V_{2}:=e_{2} ;\right.$ unit $)$.

Binary operations are evaluated from left to right (for binop see Section 2.1.2).

The lengthiest rule is the one for method call. Its reading is easy: Evaluate $e$ to an address $a$ and the parameter list $p s$ to a list of values $v s,{ }^{1}$ look up the class $C$ of the object in the heap at $a$, look up the parameter names pns and the body body of the method $M$ visible from $C$, and evaluate the body in a store that maps this to Addr a and the formal parameter names to the actual parameter values (having made sure that $v s$ and pns have the same length). The final store is the one obtained from the evaluation of the parameters-the one obtained from the evaluation of body is discarded. This rule reflects a well-formedness condition imposed later on: There are no global variables in Jinja (just as in

${ }^{1}[\Rightarrow]$ is evaluation extended to lists of expressions. Saying that the result is of the form map Val $v s$ is a declarative way of ensuring that it is a list of values and of obtaining the actual value list $v s$ (as opposed to an expression list). 


$$
\begin{aligned}
& \frac{\text { new-Addr } h=\lfloor a\rfloor \quad P \vdash C \text { has-fields FDTs } \quad h^{\prime}=h(a \mapsto(C, \text { init-fields FDTs }))}{P \vdash\langle\text { new } C,(h, l)\rangle \Rightarrow\left\langle a d d r a,\left(h^{\prime}, l\right)\right\rangle} \\
& \frac{P \vdash\left\langle e, s_{0}\right\rangle \Rightarrow\langle a d d r a,(h, l)\rangle \quad h a=\lfloor(D, f s)\rfloor \quad P \vdash D \preceq \preceq^{*} C}{P \vdash\left\langle\text { Cast } C e, s_{0}\right\rangle \Rightarrow\langle a d d r a,(h, l)\rangle} \\
& \frac{P \vdash\left\langle e, s_{0}\right\rangle \Rightarrow\left\langle\text { null, } s_{1}\right\rangle}{P \vdash\left\langle\text { Cast } C \text { e }, s_{0}\right\rangle \Rightarrow\left\langle\text { null }, s_{1}\right\rangle} \\
& P \vdash\langle\text { Val } v, s\rangle \Rightarrow\langle\text { Val } v, s\rangle \\
& \frac{l V=\lfloor v\rfloor}{P \vdash\langle\operatorname{Var} V,(h, l)\rangle \Rightarrow\langle\operatorname{Val} v,(h, l)\rangle} \\
& \frac{P \vdash\left\langle e, s_{0}\right\rangle \Rightarrow\langle\text { Val } v,(h, l)\rangle \quad l^{\prime}=l(V \mapsto v)}{P \vdash\left\langle V:=e, s_{0}\right\rangle \Rightarrow\left\langle\text { unit },\left(h, l^{\prime}\right)\right\rangle} \\
& \frac{P \vdash\left\langle e, s_{0}\right\rangle \Rightarrow\langle a d d r a,(h, l)\rangle \quad h a=\lfloor(C, f s)\rfloor \quad f s(F, D)=\lfloor v\rfloor}{P \vdash\left\langle e . F\{D\}, s_{0}\right\rangle \Rightarrow\langle\operatorname{Val} v,(h, l)\rangle} \\
& P \vdash\left\langle e_{1}, s_{0}\right\rangle \Rightarrow\left\langle a d d r a, s_{1}\right\rangle \quad P \vdash\left\langle e_{2}, s_{1}\right\rangle \Rightarrow\left\langle\operatorname{Val} v,\left(h_{2}, l_{2}\right)\right\rangle \\
& \frac{h_{2} a=\lfloor(C, f s)\rfloor \quad f^{\prime}=f s((F, D) \mapsto v) \quad h_{2}{ }^{\prime}=h_{2}\left(a \mapsto\left(C, f s^{\prime}\right)\right)}{P \vdash\left\langle e_{1} . F\{D\}:=e_{2}, s_{0}\right\rangle \Rightarrow\left\langle\text { unit },\left(h_{2}{ }^{\prime}, l_{2}\right)\right\rangle} \\
& \frac{P \vdash\left\langle e_{1}, s_{0}\right\rangle \Rightarrow\left\langle\operatorname{Val} v_{1}, s_{1}\right\rangle \quad P \vdash\left\langle e_{2}, s_{1}\right\rangle \Rightarrow\left\langle\operatorname{Val} v_{2}, s_{2}\right\rangle \quad \text { binop }\left(\text { bop }, v_{1}, v_{2}\right)=\lfloor v\rfloor}{P \vdash\left\langle e_{1} \ll \text { bop } \gg e_{2}, s_{0}\right\rangle \Rightarrow\left\langle\text { Val } v, s_{2}\right\rangle} \\
& P \vdash\left\langle e, s_{0}\right\rangle \Rightarrow\left\langle a d d r a, s_{1}\right\rangle \quad P \vdash\left\langle p s, s_{1}\right\rangle[\Rightarrow]\left\langle\operatorname{map} \text { Val } v s,\left(h_{2}, l_{2}\right)\right\rangle \\
& h_{2} a=\lfloor(C, f s)\rfloor \quad P \vdash C \text { sees } M: T s \rightarrow T=(p n s, \text { body }) \text { in } D \\
& \frac{|v s|=\mid \text { pns } \mid \quad l_{2}{ }^{\prime}=[\text { this } \mapsto \text { Addra, pns }[\mapsto] v s] \quad P \vdash\left\langle\text { body },\left(h_{2}, l_{2}{ }^{\prime}\right)\right\rangle \Rightarrow\left\langle e^{\prime},\left(h_{3}, l_{3}\right)\right\rangle}{P \vdash\left\langle e . M(p s), s_{0}\right\rangle \Rightarrow\left\langle e^{\prime},\left(h_{3}, l_{2}\right)\right\rangle} \\
& \frac{P \vdash\left\langle e_{0},\left(h_{0}, l_{0}(V:=N o n e)\right)\right\rangle \Rightarrow\left\langle e_{1},\left(h_{1}, l_{1}\right)\right\rangle}{P \vdash\left\langle\left\{V: T ; e_{0}\right\},\left(h_{0}, l_{0}\right)\right\rangle \Rightarrow\left\langle e_{1},\left(h_{1}, l_{1}\left(V:=l_{0} V\right)\right)\right\rangle} \\
& \frac{P \vdash\left\langle e_{0}, s_{0}\right\rangle \Rightarrow\left\langle\operatorname{Val} v, s_{1}\right\rangle \quad P \vdash\left\langle e_{1}, s_{1}\right\rangle \Rightarrow\left\langle e_{2}, s_{2}\right\rangle}{P \vdash\left\langle e_{0} ; e_{1}, s_{0}\right\rangle \Rightarrow\left\langle e_{2}, s_{2}\right\rangle} \\
& \frac{P \vdash\left\langle e, s_{0}\right\rangle \Rightarrow\left\langle\text { true }, s_{1}\right\rangle \quad P \vdash\left\langle e_{1}, s_{1}\right\rangle \Rightarrow\left\langle e^{\prime}, s_{2}\right\rangle}{P \vdash\left\langle\text { if }(e) e_{1} \text { else } e_{2}, s_{0}\right\rangle \Rightarrow\left\langle e^{\prime}, s_{2}\right\rangle} \\
& \frac{P \vdash\left\langle e, s_{0}\right\rangle \Rightarrow\left\langle\text { false }, s_{1}\right\rangle \quad P \vdash\left\langle e_{2}, s_{1}\right\rangle \Rightarrow\left\langle e^{\prime}, s_{2}\right\rangle}{P \vdash\left\langle\text { if }(e) e_{1} \text { else } e_{2}, s_{0}\right\rangle \Rightarrow\left\langle e^{\prime}, s_{2}\right\rangle} \\
& \frac{P \vdash\left\langle e, s_{0}\right\rangle \Rightarrow\left\langle\text { false }, s_{1}\right\rangle}{P \vdash\left\langle\text { while }(e) c, s_{0}\right\rangle \Rightarrow\left\langle\text { unit }, s_{1}\right\rangle} \\
& \frac{P \vdash\left\langle e, s_{0}\right\rangle \Rightarrow\left\langle\text { true }, s_{1}\right\rangle \quad P \vdash\left\langle c, s_{1}\right\rangle \Rightarrow\left\langle\text { Val } v_{1}, s_{2}\right\rangle \quad P \vdash\left\langle\text { while }(e) c, s_{2}\right\rangle \Rightarrow\left\langle e_{3}, s_{3}\right\rangle}{P \vdash\left\langle\text { while }(e) c, s_{0}\right\rangle \Rightarrow\left\langle e_{3}, s_{3}\right\rangle} \\
& P \vdash\langle[], s\rangle[\Rightarrow]\langle[], s\rangle \quad \frac{P \vdash\left\langle e, s_{0}\right\rangle \Rightarrow\left\langle\operatorname{Val} v, s_{1}\right\rangle \quad P \vdash\left\langle e s, s_{1}\right\rangle[\Rightarrow]\left\langle e s^{\prime}, s_{2}\right\rangle}{P \vdash\left\langle e \cdot e s, s_{0}\right\rangle[\Rightarrow]\left\langle\operatorname{Val} v \cdot e s^{\prime}, s_{2}\right\rangle}
\end{aligned}
$$

Fig. 3. Normal evaluation of expressions. 
Java, but contrary to, say, $\mathrm{C}++$ ), that is, a method body should only refer to this and its parameters.

In Jinja, blocks with local variables, sequential composition, conditional and while-loops, are expressions too, in contrast to Java, where they are commands and do not return a value. In a block, the expression is evaluated in the context of a store where the local variable has been removed, that is, set to None. Afterwards, the original value of the variable in the initial store is restored. Sequential composition discards the value of the first expression. Similarly, while-loops discard the value of their body and, upon termination, return unit.

The rules for $[\Rightarrow]$, the evaluation of expression lists (needed for method call), define that lists are evaluated from left to right. This concludes the complete semantics of the exception-free fragment of Jinja.

2.2.4 Exceptions. The aforementioned rules assume that during evaluation everything fits together. If it does not, the semantics gets stuck, that is, there is no final value. For example, evaluation of $\langle\operatorname{Var} V,(h, l)\rangle$ only succeeds if $V \in d o m ~ l$. Later on (Section 2.7), a static analysis ("definite assignment") will identify expressions where $V \in d o m l$ always holds. Thus we do not need a rule for the situation where $V \notin d o m ~ l$. In contrast, many exceptional situations arise because of null references which we deal with by raising an exception. In other words, the expression does not evaluate to a normal value but to an exception throw $(a d d r a$ ), where $a$ is the address of some object, the exception object.

There are both system and user exceptions. User exceptions can refer to arbitrary objects. System exceptions refer to an object in one of the system exception classes:

sys-xcpts $\equiv\{$ NullPointer, ClassCast, OutOfMemory $\}$

Their names speak for themselves. Since system exception objects do not carry any information in addition to their class name, we can simplify their treatment by preallocating one object for each system exception class. Thus, a few addresses are reserved for preallocated system exception objects. This is modelled by a function $a d d r$-of-sys-xcpt :: cname $\Rightarrow a d d r$ whose precise definition is not important. To ease notation we introduce some abbreviations:

$$
\begin{array}{lll}
\text { Throw } a & \equiv \text { throw }(a d d r a) \\
\text { THROW } C & \equiv \text { Throw }(a d d r \text {-of-sys-xcpt } C \text { ) }
\end{array}
$$

2.2.5 Exceptional Evaluation. The basic rules for throwing and catching exceptions are shown in Figure 4. In the following situations, system exceptions are thrown: if there is no more free storage, if a cast fails, or if the object reference in a field access, field update, or method call is null. The throw construct may throw any expression of class type, which is a simplification of Java's exceptions. Throwing null leads to a NullPointer exception.

Note that we have maintained Java's eager evaluation scheme of evaluating all subterms before throwing any system exception. This permits a simple compilation strategy where the values of the subterms are first put on the stack 


$$
\begin{aligned}
& \frac{n e w-A d d r h=\text { None }}{P \vdash\langle\text { new } C,(h, l)\rangle \Rightarrow\langle\text { THROW OutOfMemory },(h, l)\rangle} \\
& \frac{P \vdash\left\langle e, s_{0}\right\rangle \Rightarrow\langle\text { addr } a,(h, l)\rangle \quad h a=\lfloor(D, f s)\rfloor \quad \neg P \vdash D \preceq{ }^{*} C}{P \vdash\left\langle\text { Cast } C e, s_{0}\right\rangle \Rightarrow\langle\text { THROW ClassCast },(h, l)\rangle} \\
& \frac{P \vdash\left\langle e, s_{0}\right\rangle \Rightarrow\left\langle\text { null }, s_{1}\right\rangle}{P \vdash\left\langle e . F\{D\}, s_{0}\right\rangle \Rightarrow\left\langle\text { THROW NullPointer }, s_{1}\right\rangle} \\
& \frac{P \vdash\left\langle e_{1}, s_{0}\right\rangle \Rightarrow\left\langle\text { null }, s_{1}\right\rangle \quad P \vdash\left\langle e_{2}, s_{1}\right\rangle \Rightarrow\left\langle\text { Val } v, s_{2}\right\rangle}{P \vdash\left\langle e_{1} . F\{D\}:=e_{2}, s_{0}\right\rangle \Rightarrow\left\langle\text { THROW NullPointer }, s_{2}\right\rangle} \\
& \frac{P \vdash\left\langle e, s_{0}\right\rangle \Rightarrow\left\langle\text { null }, s_{1}\right\rangle \quad P \vdash\left\langle p s, s_{1}\right\rangle[\Rightarrow]\left\langle\text { map Val vs, } s_{2}\right\rangle}{P \vdash\left\langle e \cdot M(p s), s_{0}\right\rangle \Rightarrow\left\langle\text { THROW NullPointer }, s_{2}\right\rangle} \\
& \frac{P \vdash\left\langle e, s_{0}\right\rangle \Rightarrow\left\langle a d d r a, s_{1}\right\rangle}{P \vdash\left\langle\text { throw } e, s_{0}\right\rangle \Rightarrow\left\langle\text { Throw } a, s_{1}\right\rangle} \quad \frac{P \vdash\left\langle e, s_{0}\right\rangle \Rightarrow\left\langle\text { null }, s_{1}\right\rangle}{P \vdash\left\langle\text { throw } e, s_{0}\right\rangle \Rightarrow\left\langle\text { THROW NullPointer }, s_{1}\right\rangle} \\
& \frac{P \vdash\left\langle e_{1}, s_{0}\right\rangle \Rightarrow\left\langle\text { Val } v_{1}, s_{1}\right\rangle}{P \vdash\left\langle\operatorname{try} e_{1} \operatorname{catch}(C V) e_{2}, s_{0}\right\rangle \Rightarrow\left\langle\text { Val } v_{1}, s_{1}\right\rangle} \\
& P \vdash\left\langle e_{1}, s_{0}\right\rangle \Rightarrow\left\langle\text { Throw } a,\left(h_{1}, l_{1}\right)\right\rangle \\
& \frac{h_{1} a=\lfloor(D, f s)\rfloor \quad P \vdash D \preceq{ }^{*} C \quad P \vdash\left\langle e_{2},\left(h_{1}, l_{1}(V \mapsto A d d r a)\right)\right\rangle \Rightarrow\left\langle e_{2}{ }^{\prime},\left(h_{2}, l_{2}\right)\right\rangle}{P \vdash\left\langle\operatorname{try} e_{1} \operatorname{catch}(C V) e_{2}, s_{0}\right\rangle \Rightarrow\left\langle e_{2}{ }^{\prime},\left(h_{2}, l_{2}\left(V:=l_{1} V\right)\right)\right\rangle} \\
& \frac{P \vdash\left\langle e_{1}, s_{0}\right\rangle \Rightarrow\left\langle\text { Throw } a,\left(h_{1}, l_{1}\right)\right\rangle \quad h_{1} a=\lfloor(D, f s)\rfloor \quad \neg P \vdash D \preceq \preceq^{*} C}{P \vdash\left\langle\operatorname{try} e_{1} \operatorname{catch}(C V) e_{2}, s_{0}\right\rangle \Rightarrow\left\langle\text { Throw } a,\left(h_{1}, l_{1}\right)\right\rangle}
\end{aligned}
$$

Fig. 4. Throwing and catching exceptions.

unchecked, and the check is performed at the end by the machine instruction, for example, field access, accessing the object reference in question.

Thrown exceptions can be caught using the construct try $e_{1}$ catch $(C V) e_{2}$. If $e_{1}$ evaluates to a value, the whole expression evaluates to that value. If $e_{1}$ evaluates to an exception throw $a$ such that $a$ refers to an object of a subclass of $C, V$ is set to Addr $a$ and $e_{2}$ is evaluated; otherwise throw $a$ is the result of the evaluation.

Finally, exceptions must be propagated. That is, if the evaluation of a certain subexpression throws an exception, the evaluation of the whole expression has to throw that exception. The exception propagation rules are straightforward and shown in Figure 5. This concludes the exposition of the evaluation rules.

A compact representation of the exception propagation rules can be achieved by introducing the notion of a context $C_{x}$ (essentially a grammar for positions in expressions where exceptions propagate to the top), and by giving one rule

$$
\frac{P \vdash\langle e, s\rangle \Rightarrow\left\langle\text { throw } e^{\prime}, s^{\prime}\right\rangle}{P \vdash\left\langle C_{x}[e], s\right\rangle \Rightarrow\left\langle\text { throw } e^{\prime}, s^{\prime}\right\rangle} .
$$

We prefer not to formalize these additional notions and to stay within a fixed basic framework of ordinary expressions.

2.2.6 Final Expressions. Now that we have the complete set of rules, we can show that evaluation always produces a final expression:

$$
\text { final } e \equiv(\exists v \cdot e=\operatorname{Val} v) \vee(\exists a . e=\text { throw } a)
$$




$$
\begin{aligned}
& \frac{P \vdash\left\langle e, s_{0}\right\rangle \Rightarrow\left\langle\text { throw } e^{\prime}, s_{1}\right\rangle}{P \vdash\left\langle\text { Cast } C e, s_{0}\right\rangle \Rightarrow\left\langle\text { throw } e^{\prime}, s_{1}\right\rangle} \quad \frac{P \vdash\left\langle e, s_{0}\right\rangle \Rightarrow\left\langle\text { throw } e^{\prime}, s_{1}\right\rangle}{P \vdash\left\langle V:=e, s_{0}\right\rangle \Rightarrow\left\langle\text { throw } e^{\prime}, s_{1}\right\rangle} \\
& \frac{P \vdash\left\langle e, s_{0}\right\rangle \Rightarrow\left\langle\text { throw } e^{\prime}, s_{1}\right\rangle}{P \vdash\left\langle e . F\{D\}, s_{0}\right\rangle \Rightarrow\left\langle\text { throw } e^{\prime}, s_{1}\right\rangle} \quad \frac{P \vdash\left\langle e_{1}, s_{0}\right\rangle \Rightarrow\left\langle\text { throw } e^{\prime}, s_{1}\right\rangle}{P \vdash\left\langle e_{1} . F\{D\}:=e_{2}, s_{0}\right\rangle \Rightarrow\left\langle\text { throw } e^{\prime}, s_{1}\right\rangle} \\
& \frac{P \vdash\left\langle e_{1}, s_{0}\right\rangle \Rightarrow\left\langle\text { Val } v, s_{1}\right\rangle \quad P \vdash\left\langle e_{2}, s_{1}\right\rangle \Rightarrow\left\langle\text { throw } e^{\prime}, s_{2}\right\rangle}{P \vdash\left\langle e_{1} . F\{D\}:=e_{2}, s_{0}\right\rangle \Rightarrow\left\langle\text { throw } e^{\prime}, s_{2}\right\rangle} \\
& \frac{P \vdash\left\langle e_{1}, s_{0}\right\rangle \Rightarrow\left\langle\text { throw } e, s_{1}\right\rangle}{P \vdash\left\langle e_{1} \ll \text { bop } \gg e_{2}, s_{0}\right\rangle \Rightarrow\left\langle\text { throw } e, s_{1}\right\rangle} \\
& \frac{P \vdash\left\langle e_{1}, s_{0}\right\rangle \Rightarrow\left\langle\operatorname{Val} v_{1}, s_{1}\right\rangle \quad P \vdash\left\langle e_{2}, s_{1}\right\rangle \Rightarrow\left\langle\text { throw } e, s_{2}\right\rangle}{P \vdash\left\langle e_{1} \ll \text { bop } \gg e_{2}, s_{0}\right\rangle \Rightarrow\left\langle\text { throw } e, s_{2}\right\rangle} \\
& \frac{P \vdash\left\langle e, s_{0}\right\rangle \Rightarrow\left\langle\text { throw } e^{\prime}, s_{1}\right\rangle}{P \vdash\left\langle e . M(p s), s_{0}\right\rangle \Rightarrow\left\langle\text { throw } e^{\prime}, s_{1}\right\rangle} \\
& \frac{P \vdash\left\langle e, s_{0}\right\rangle \Rightarrow\left\langle\operatorname{Val} v, s_{1}\right\rangle \quad P \vdash\left\langle e s, s_{1}\right\rangle[\Rightarrow]\left\langle\operatorname{map} \operatorname{Val} v s @\left(\text { throw ex } \cdot e s^{\prime}\right), s_{2}\right\rangle}{P \vdash\left\langle e \cdot M(e s), s_{0}\right\rangle \Rightarrow\left\langle\text { throw ex }, s_{2}\right\rangle} \\
& \frac{P \vdash\left\langle e_{0}, s_{0}\right\rangle \Rightarrow\left\langle\text { throw } e, s_{1}\right\rangle}{P \vdash\left\langle e_{0} ; e_{1}, s_{0}\right\rangle \Rightarrow\left\langle\text { throw } e, s_{1}\right\rangle} \quad \frac{P \vdash\left\langle e, s_{0}\right\rangle \Rightarrow\left\langle\text { throw } e^{\prime}, s_{1}\right\rangle}{P \vdash\left\langle\text { if }(e) e_{1} \text { else } e_{2}, s_{0}\right\rangle \Rightarrow\left\langle\text { throw } e^{\prime}, s_{1}\right\rangle} \\
& \frac{P \vdash\left\langle e, s_{0}\right\rangle \Rightarrow\left\langle\text { throw } e^{\prime}, s_{1}\right\rangle}{P \vdash\left\langle\text { while }(e) c, s_{0}\right\rangle \Rightarrow\left\langle\text { throw } e^{\prime}, s_{1}\right\rangle} \frac{P \vdash\left\langle e, s_{0}\right\rangle \Rightarrow\left\langle\text { true }, s_{1}\right\rangle \quad P \vdash\left\langle c, s_{1}\right\rangle \Rightarrow\left\langle\text { throw } e^{\prime}, s_{2}\right\rangle}{P \vdash\left\langle\text { while }(e) c, s_{0}\right\rangle \Rightarrow\left\langle\text { throw } e^{\prime}, s_{2}\right\rangle} \\
& \frac{P \vdash\left\langle e, s_{0}\right\rangle \Rightarrow\left\langle\text { throw } e^{\prime}, s_{1}\right\rangle}{P \vdash\left\langle\text { throw } e, s_{0}\right\rangle \Rightarrow\left\langle\text { throw } e^{\prime}, s_{1}\right\rangle} \quad \frac{P \vdash\left\langle e, s_{0}\right\rangle \Rightarrow\left\langle\text { throw } e^{\prime}, s_{1}\right\rangle}{P \vdash\left\langle e \cdot e s, s_{0}\right\rangle[\Rightarrow]\left\langle\text { throw } e^{\prime} \cdot e s, s_{1}\right\rangle}
\end{aligned}
$$

Fig. 5. Exception propagation.

Lemma 2.1. If $P \vdash\langle e, s\rangle \Rightarrow\left\langle e^{\prime}, s\right\rangle$, then final $e^{\prime}$.

The proof is by induction on the evaluation relation $\Rightarrow$. Since the latter is defined simultaneously with the evaluation relation $[\Rightarrow]$ for expression lists, we need to prove a proposition about $[\Rightarrow]$ simultaneously with Lemma 2.1. This will also be the common proof pattern in all other inductive proofs about $\Rightarrow$. In most cases the statement about $[\Rightarrow]$ is a lifted version of the one about $\Rightarrow$. In the above case, we might expect something like $P \vdash\langle e s, s\rangle[\Rightarrow]\left\langle e s^{\prime}, s^{\prime}\right\rangle \Longrightarrow$ $\forall e^{\prime} \in$ set es'. final $e^{\prime}$. However, this is wrong: due to exceptions, evaluation may stop before the end of the list. A final expression list is a list of values, possibly followed by a throw and some further expressions:

finals es $\equiv(\exists v s . e s=m a p \operatorname{Val} v s) \vee\left(\exists v s a e^{\prime} . e s=m a p \operatorname{Val} v s @\left(\right.\right.$ throw $\left.\left.a \cdot e s^{\prime}\right)\right)$,

and Lemma 2.1 for lists is simply "If $P \vdash\langle e s, s\rangle[\Rightarrow]\left\langle e s^{\prime}, s^{\prime}\right\rangle$ then finals es'."

It is equally straightforward to prove that final expressions evaluate to themselves:

Lemma 2.2. If final e then $P \vdash\langle e, s\rangle \Rightarrow\langle e, s\rangle$.

Of course, an analogous lemma holds for expression lists, but we have chosen not to show it. We will follow this practice whenever the list version of a theorem is obvious. In fact, we could dispose of expression lists altogether by restricting Jinja methods to a single parameter. However, this is precisely the kind of simplification we do not want to make because it would give 
the wrong impression that including expression lists could be a significant burden.

\subsection{Small Step Semantics}

Because of its simplicity, a big step semantics has several drawbacks. For example, it cannot accommodate parallelism, a potentially desirable extension of Jinja. The reason is that $\Rightarrow$ cannot talk about intermediate states during evaluation. For the same reason, the type safety proof in Section 2.9 needs a finer-grained semantics. Otherwise, we cannot prove that type-correct expressions do not get stuck during evaluation because the big step semantics does not distinguish between divergence (of nonterminating expressions) and deadlock (of ill-typed expressions). Thus we now move to an equivalent small step semantics.

The judgement for the small step semantics is $P \vdash\langle e, s\rangle \rightarrow\left\langle e^{\prime}, s^{\prime}\right\rangle$ and describes a single microstep in the reduction of $e$ towards its final value. We say that $e$ reduces to $e^{\prime}$ (in one step). In the following, we will compose sequences of such single steps $\left\langle e_{1}, s_{1}\right\rangle \rightarrow\left\langle e_{2}, s_{2}\right\rangle \ldots \rightarrow\left\langle e_{n}, s_{n}\right\rangle$ to reduce an expression completely.

As for the big step semantics, we can define normal and exceptional reductions separately. We begin with normal reductions. The rules come in two flavours: those that reduce a subexpression of an expression and those that reduce the whole expression. The former have no counterpart in the big step semantics, as they are handled implicitly in the premises of the big step rules.

2.3.1 Subexpression Reduction. These rules essentially describe the order in which subexpressions are evaluated. Therefore most of them follow a common pattern:

$$
\frac{P \vdash\langle e, s\rangle \rightarrow\left\langle e^{\prime}, s^{\prime}\right\rangle}{P \vdash\langle c \ldots e \ldots, s\rangle \rightarrow\left\langle c \ldots e^{\prime} \ldots, s^{\prime}\right\rangle},
$$

where $c$ is a constructor and $e$ and $e^{\prime}$ are metavariables. The other subexpressions of $c$ may be more complex to indicate, for example, which of them must be values already, thus expressing the order of reduction. The rules for Jinja subexpression reduction are shown in Figure 6. The initial ones follow the previous pattern exactly. For example, the rules for field assignment express that the lefthand side is evaluated before the righthand side.

The rules for blocks are more complicated. In a block $\{V: T ; e\}$ we keep reducing $e$ in a store where $V$ is undefined (None), restoring the original binding of $V$ after each step. Once the store after the reduction step binds $V$ to a value $v$, this binding is remembered by adding an assignment in front of the reduced expression, yielding $\left\{V: T ; V:=\operatorname{Val} v ; e^{\prime}\right\}$. The final rule reduces such blocks. This additional rule is necessary because $\{V: T ; V:=$ Val $v ; e\}$ must not be reduced as before, by reducing all of $V:=\operatorname{Val} v$; $e$ to $e$ (thus losing the binding for $V$ ), but by reducing $e$ directly. To this end we have introduced the predicate

$$
\text { assigned } V e \equiv \exists v e^{\prime} . e=V:=\mathrm{Val} v ; e^{\prime}
$$

and added its negation as a precondition to the initial two reduction rules. 


$$
\begin{aligned}
& \frac{P \vdash\langle e, s\rangle \rightarrow\left\langle e^{\prime}, s^{\prime}\right\rangle}{P \vdash\langle\text { Cast } C e, s\rangle \rightarrow\left\langle\text { Cast } C e^{\prime}, s^{\prime}\right\rangle} \quad \frac{P \vdash\langle e, s\rangle \rightarrow\left\langle e^{\prime}, s^{\prime}\right\rangle}{P \vdash\langle V:=e, s\rangle \rightarrow\left\langle V:=e^{\prime}, s^{\prime}\right\rangle} \\
& \frac{P \vdash\langle e, s\rangle \rightarrow\left\langle e^{\prime}, s^{\prime}\right\rangle}{P \vdash\langle e . F\{D\}, s\rangle \rightarrow\left\langle e^{\prime} . F\{D\}, s^{\prime}\right\rangle} \quad \frac{P \vdash\langle e, s\rangle \rightarrow\left\langle e^{\prime}, s^{\prime}\right\rangle}{P \vdash\left\langle e . F\{D\}:=e_{2}, s\right\rangle \rightarrow\left\langle e^{\prime} . F\{D\}:=e_{2}, s^{\prime}\right\rangle} \\
& \frac{P \vdash\langle e, s\rangle \rightarrow\left\langle e^{\prime}, s^{\prime}\right\rangle}{P \vdash\langle\text { Val v.F }\{D\}:=e, s\rangle \rightarrow\left\langle\operatorname{Val} v . F\{D\}:=e^{\prime}, s^{\prime}\right\rangle} \quad \frac{P \vdash\langle e, s\rangle \rightarrow\left\langle e^{\prime}, s^{\prime}\right\rangle}{P \vdash\left\langle e \ll b o p \gg e_{2}, s\right\rangle \rightarrow\left\langle e^{\prime} \ll b o p \gg e_{2}, s^{\prime}\right\rangle} \\
& \frac{P \vdash\langle e, s\rangle \rightarrow\left\langle e^{\prime}, s^{\prime}\right\rangle}{P \vdash\left\langle\text { Val } v_{1} \ll b o p \gg e, s\right\rangle \rightarrow\left\langle\text { Val } v_{1} \ll b o p \gg e^{\prime}, s^{\prime}\right\rangle} \\
& \frac{P \vdash\langle e,(h, l(V:=\text { None }))\rangle \rightarrow\left\langle e^{\prime},\left(h^{\prime}, l^{\prime}\right)\right\rangle \quad l^{\prime} V=\text { None } \neg \text { assigned } V e}{P \vdash\langle\{V: T ; e\},(h, l)\rangle \rightarrow\left\langle\left\{V: T ; e^{\prime}\right\},\left(h^{\prime}, l^{\prime}(V:=l V)\right)\right\rangle} \\
& \frac{P \vdash\langle e,(h, l(V:=\text { None }))\rangle \rightarrow\left\langle e^{\prime},\left(h^{\prime}, l^{\prime}\right)\right\rangle \quad l^{\prime} V=\lfloor v\rfloor \quad \neg \text { assigned } V e}{P \vdash\langle\{V: T ; e\},(h, l)\rangle \rightarrow\left\langle\left\{V: T ; V:=\operatorname{Val} v ; e^{\prime}\right\},\left(h^{\prime}, l^{\prime}(V:=l V)\right)\right\rangle} \\
& \frac{P \vdash\langle e,(h, l(V \mapsto v))\rangle \rightarrow\left\langle e^{\prime},\left(h^{\prime}, l^{\prime}\right)\right\rangle \quad l^{\prime} V=\left\lfloor v^{\prime}\right\rfloor}{P \vdash\langle\{V: T ; V:=\operatorname{Val} v ; e\},(h, l)\rangle \rightarrow\left\langle\left\{V: T ; V:=\operatorname{Val} v^{\prime} ; e^{\prime}\right\},\left(h^{\prime}, l^{\prime}(V:=l V)\right)\right\rangle} \\
& \frac{P \vdash\langle e, s\rangle \rightarrow\left\langle e^{\prime}, s^{\prime}\right\rangle}{P \vdash\langle e . M(e s), s\rangle \rightarrow\left\langle e^{\prime} \cdot M(e s), s^{\prime}\right\rangle} \quad \frac{P \vdash\langle e s, s\rangle[\rightarrow]\left\langle e s^{\prime}, s^{\prime}\right\rangle}{P \vdash\langle\operatorname{Val} v \cdot M(e s), s\rangle \rightarrow\left\langle\operatorname{Val} v \cdot M\left(e s^{\prime}\right), s^{\prime}\right\rangle} \\
& \frac{P \vdash\langle e, s\rangle \rightarrow\left\langle e^{\prime}, s^{\prime}\right\rangle}{P \vdash\left\langle e ; e_{2}, s\right\rangle \rightarrow\left\langle e^{\prime} ; e_{2}, s^{\prime}\right\rangle} \quad \frac{P \vdash\langle e, s\rangle \rightarrow\left\langle e^{\prime}, s^{\prime}\right\rangle}{P \vdash\left\langle\text { if }(e) e_{1} \text { else } e_{2}, s\right\rangle \rightarrow\left\langle\text { if }\left(e^{\prime}\right) e_{1} \text { else } e_{2}, s^{\prime}\right\rangle} \\
& \frac{P \vdash\langle e, s\rangle \rightarrow\left\langle e^{\prime}, s^{\prime}\right\rangle}{P \vdash\langle e \cdot e s, s\rangle[\rightarrow]\left\langle e^{\prime} \cdot e s, s^{\prime}\right\rangle} \quad \frac{P \vdash\langle e s, s\rangle[\rightarrow]\left\langle e s^{\prime}, s^{\prime}\right\rangle}{P \vdash\langle\text { Val } v \cdot e s, s\rangle[\rightarrow]\left\langle\text { Val } v \cdot e s^{\prime}, s^{\prime}\right\rangle}
\end{aligned}
$$

Fig. 6. Subexpression reduction.

Note that we cannot simply treat local variables by creating "new" variables because we do not know which other variables exist in the context; dom $l$ does not contain all of them because variables need not be initialized upon creation.

To reduce a method call, the object expression is reduced until it has become an address, and then the parameters are reduced. The relation $[\rightarrow]$ is the extension of $\rightarrow$ to expression lists, which are reduced from left to right, and each element is reduced until it has become a value.

2.3.2 Expression Reduction. Once the subexpressions are sufficiently reduced, we can reduce the whole expression. The rules are shown in Figure 7. Most of the rules are fairly intuitive and many resemble their big step counterparts. The only one that deserves some explanation is the one for method invocation. In order to avoid explicit stacks we use local variables to hold the values of the parameters. The required nested block structure is built with the help of the auxiliary function blocks of type vname list $\times$ ty list $\times$ val list $\times$ expr $\Rightarrow$ expr:

blocks $(V \cdot V s, T \cdot T s, v \cdot v s, e)=\{V: T ; V:=\operatorname{Val} v ;$ blocks $(V s, T s, v s, e)\}$

blocks ([], [], [],e) $=e$

Note that we can only get away with this simple rule for method call because there are no global variables in Java. Otherwise, we could unfold a method body that refers to some global variable into a context that declares a local variable of the same name, which would essentially amount to dynamic variable binding. 


$$
\begin{aligned}
& \frac{n e w-A d d r h=\lfloor a\rfloor \quad P \vdash C \text { has-fields FDTs }}{P \vdash\langle\text { new } C,(h, l)\rangle \rightarrow\langle a d d r a,(h(a \mapsto(C, \text { init-fields FDTs })), l)\rangle} \\
& \frac{h p s a=\lfloor(D, f s)\rfloor \quad P \vdash D \preceq * C}{P \vdash\langle\text { Cast } C(\text { addr } a), s\rangle \rightarrow\langle a d d r a, s\rangle} \quad P \vdash\langle\text { Cast } C \text { null }, s\rangle \rightarrow\langle\text { null }, s\rangle \\
& \frac{l c l s V=\lfloor v\rfloor}{P \vdash\langle\operatorname{Var} V, s\rangle \rightarrow\langle\operatorname{Val} v, s\rangle} \quad P \vdash\langle V:=\operatorname{Val} v,(h, l)\rangle \rightarrow\langle\text { unit },(h, l(V \mapsto v))\rangle \\
& \frac{\text { binop }\left(\text { bop }, v_{1}, v_{2}\right)=\lfloor v\rfloor}{P \vdash\left\langle\text { Val } v_{1} \ll b o p \gg \operatorname{Val} v_{2}, s\right\rangle \rightarrow\langle\text { Val } v, s\rangle} \quad \frac{h p s a=\lfloor(C, f s)\rfloor \quad f s(F, D)=\lfloor v\rfloor}{P \vdash\langle\text { addra.F }\{D\}, s\rangle \rightarrow\langle\text { Val } v, s\rangle} \\
& \frac{h a=\lfloor(C, f s)\rfloor}{P \vdash\langle a d d r a . F\{D\}:=\operatorname{Val} v,(h, l)\rangle \rightarrow\left\langle\text { unit },\left(h\left(a \mapsto\left(C, f_{s}((F, D) \mapsto v)\right)\right), l\right)\right\rangle} \\
& h p s a=\lfloor(C, f s)\rfloor \\
& \frac{P \vdash C \text { sees } M: T s \rightarrow T=(\text { pns, body }) \text { in } D \quad|v s|=|p n s| \quad|T s|=|p n s|}{P \vdash\langle\text { addr a.M(map Val vs }), s\rangle \rightarrow\langle\text { blocks }(\text { this } \cdot \text { pns, Class } D \cdot T s, \text { Addr a } \cdot \text { vs, body }), s\rangle} \\
& P \vdash\langle\{V: T ; V:=\operatorname{Val} v \text {; Val } u\}, s\rangle \rightarrow\langle\operatorname{Val} u, s\rangle \quad P \vdash\langle\{V: T ; \operatorname{Val} u\}, s\rangle \rightarrow\langle\operatorname{Val} u, s\rangle \\
& P \vdash\left\langle\operatorname{Val} v ; e_{2}, s\right\rangle \rightarrow\left\langle e_{2}, s\right\rangle \\
& P \vdash\left\langle\text { if }(\text { true }) e_{1} \text { else } e_{2}, s\right\rangle \rightarrow\left\langle e_{1}, s\right\rangle \quad P \vdash\left\langle\text { if (false) } e_{1} \text { else } e_{2}, s\right\rangle \rightarrow\left\langle e_{2}, s\right\rangle \\
& P \vdash\langle\text { while }(b) c, s\rangle \rightarrow\langle\text { if }(b)(c \text {; while }(b) c) \text { else unit, } s\rangle
\end{aligned}
$$

Fig. 7. Expression reductions.

2.3.3 Exceptional Reduction. The rules for exception throwing are shown in Figure 8. System exceptions are thrown almost exactly the same way as in the big step semantics. Expression throw $e$ is reduced by reducing $e$ as long as possible and throwing NullPointer if necessary. And this is how try $e$ catch $(C V)$ $e_{2}$ is reduced: First we must reduce $e$. If it becomes a value, the whole expression evaluates to that value. If it becomes a throw $a$, there are two possibilities: If $a$ can be caught, the term reduces to a block with $V$ set to $a$ and body $e_{2}$, otherwise, the exception is propagated. Exception propagation for all other constructs is shown in Figure 9.

It should be noted that $\{V: T$; throw $e\}$ can, in general, not be reduced to throw $e$ because $e$ may refer to the local $V$ which must not escape its scope. Hence, $e$ must be reduced to an address first.

2.3.4 The Reflexive Transitive Closure. If we write $P \vdash\left\langle e_{1}, s_{1}\right\rangle \rightarrow^{*}\left\langle e_{n}, s_{n}\right\rangle$, this means that there is a sequence of reductions $P \vdash\left\langle e_{1}, s_{1}\right\rangle \rightarrow\left\langle e_{2}, s_{2}\right\rangle, P \vdash$ $\left\langle e_{2}, s_{2}\right\rangle \rightarrow\left\langle e_{3}, s_{3}\right\rangle \ldots$, and similarly for $[\rightarrow]$ and $[\rightarrow]^{*}$.

\subsection{Well-Formedness}

We are now aiming to show that the big and small step semantics are closely related. For this (and many other proofs), we need to impose various wellformedness constraints on programs. Some of them are generic, like the constraint that the type in a field declaration must be a valid type. Others depend on the type of method bodies. To factor out the latter constraints, the wellformedness test on programs will be parameterized by a well-formedness test 


$$
\begin{aligned}
& \frac{n e w-A d d r h=\text { None }}{P \vdash\langle\text { new } C,(h, l)\rangle \rightarrow\langle\text { THROW OutOfMemory },(h, l)\rangle} \\
& \frac{h p \text { s a } a=\lfloor(D, f s)\rfloor \quad \neg P \vdash D \preceq * C}{P \vdash\langle\text { Cast } C(\text { addr } a), s\rangle \rightarrow\langle\text { THROW ClassCast }, s\rangle} \\
& P \vdash\langle\text { null.F }\{D\}, s\rangle \rightarrow\langle\text { THROW NullPointer }, s\rangle \\
& P \vdash\langle\text { null.F }\{D\}:=\text { Val } v, s\rangle \rightarrow\langle\text { THROW NullPointer }, s\rangle \\
& P \vdash\langle\text { null.M(map Val vs }), s\rangle \rightarrow\langle\text { THROW NullPointer }, s\rangle \\
& \frac{P \vdash\langle e, s\rangle \rightarrow\left\langle e^{\prime}, s^{\prime}\right\rangle}{P \vdash\langle\text { throw } e, s\rangle \rightarrow\left\langle\text { throw } e^{\prime}, s^{\prime}\right\rangle} \quad P \vdash\langle\text { throw null,s } \quad \rightarrow\langle\text { THROW NullPointer }, s\rangle \\
& \frac{P \vdash\langle e, s\rangle \rightarrow\left\langle e^{\prime}, s^{\prime}\right\rangle}{P \vdash\left\langle\operatorname{try} e \operatorname{catch}(C V) e_{2}, s\right\rangle \rightarrow\left\langle\operatorname{try} e^{\prime} \operatorname{catch}(C V) e_{2}, s^{\prime}\right\rangle} \\
& P \vdash\left\langle\operatorname{try} \text { Val } v \text { catch }(C V) e_{2}, s\right\rangle \rightarrow\langle\operatorname{Val} v, s\rangle \\
& \frac{h p s a=\lfloor(D, f s)\rfloor \quad P \vdash D \preceq{ }^{*} C}{P \vdash\left\langle\text { try Throw } a \text { catch }(C V) e_{2}, s\right\rangle \rightarrow\left\langle\left\{V: \text { Class } C ; V:=\operatorname{addr} a ; e_{2}\right\}, s\right\rangle} \\
& \frac{h p \text { s } a=\lfloor(D, f s)\rfloor \quad \neg P \vdash D \preceq{ }^{*} C}{P \vdash\left\langle\text { try Throw } a \text { catch }(C V) e_{2}, s\right\rangle \rightarrow\langle\text { Throw } a, s\rangle}
\end{aligned}
$$

Fig. 8. Exceptional expression reduction.

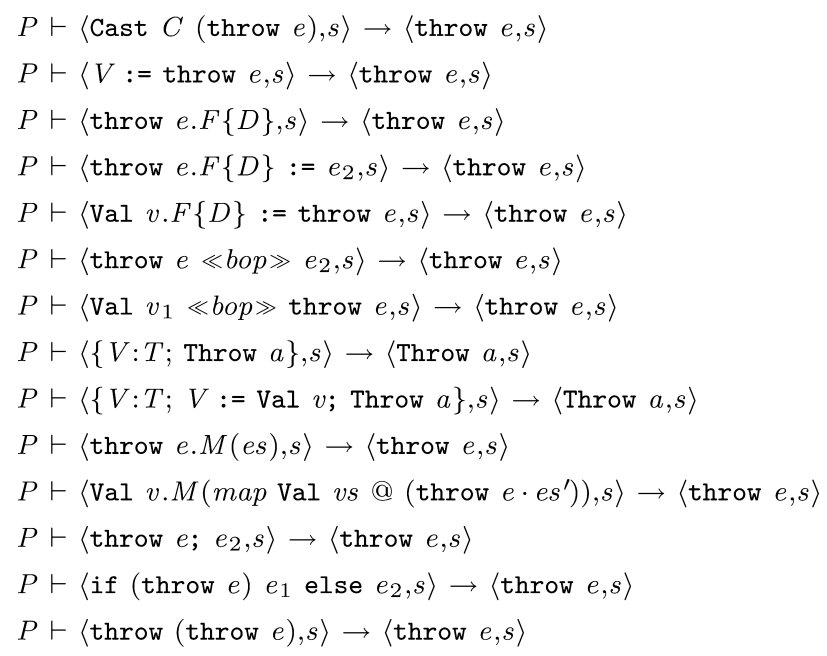

Fig. 9. Exception propagation.

for methods:

types ' $m w$ f-mdecl-test $=$ 'm prog $\Rightarrow$ cname $\Rightarrow$ 'm mdecl $\Rightarrow$ bool

Tests of this type are meant to check if a certain method declaration in a certain class within a certain program is well-formed.

Declarations are lists of pairs. In order to forbid repeated declarations of the same name, we introduce the auxiliary predicate distinct-fst, which checks 


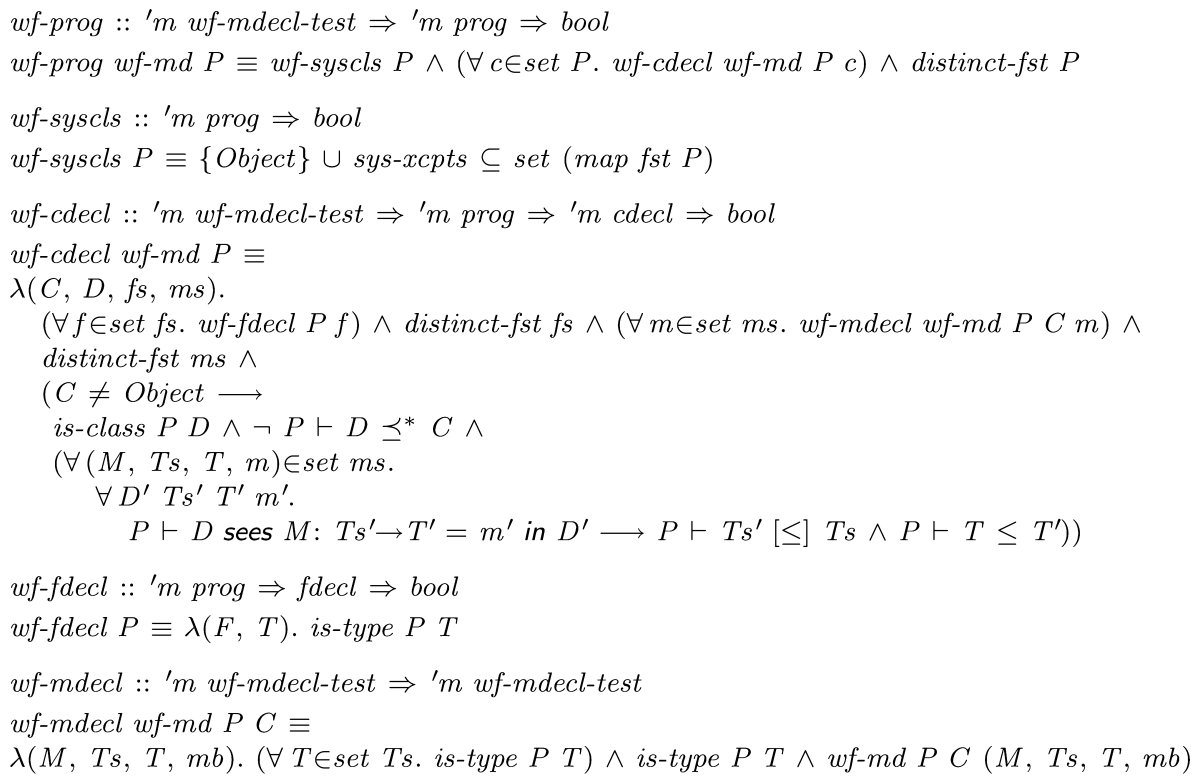

Fig. 10. Generic well-formedness.

that in a list of pairs, all first components are distinct: distinct-fst $\equiv$ distinct o map fst.

The well-formedness predicates are shown in Figure 10. They employ the following notions from Jinja's type system: Condition is-type $P T$ checks if $T$ is a valid Jinja type, $P \vdash T \leq T^{\prime}$ checks if $T$ is a subtype of $T^{\prime}$, and $P \vdash T s[\leq] T s^{\prime}$ if, element by element, $T s$ is a subtype of $T s^{\prime}$, all in the context of $P$. We will only define these notions formally in Section 2.6 because their definition is not relevant beforehand.

Let us now look at Figure 10. A program is ok (= well-formed) iff it contains all system classes (Object and all system exceptions), all class declarations are ok, and no class is declared twice. A declaration of a class $C$ is ok iff all field declarations and all method declarations are ok, no field or method is declared twice, and, if $C \neq$ Object, then its superclass $D$ exists and $D$ is not a subclass of $C$ (to rule out cycles), and method overriding is contravariant in the argument type and covariant in the result type. In other words, if $C$ overrides a method declaration visible from $D$, then the new declaration must have more specific argument types and a more general result type. Note that overriding involves only the method name-there is no overloading.

2.4.1 Weak Well-Formedness. We will now instantiate $w f$-prog with a constraint needed for relating big and small step semantics: method bodies should not refer to global variables. This requires the notion of free variables in an expression, collected by function $f v::$ expr $\Rightarrow$ vname set defined in Figure 11. A Jinja method declaration is weakly well-formed iff the following conditions hold: There are as many parameter types as parameter names, the parameter names are all distinct, this is not among the parameter names, and the free 


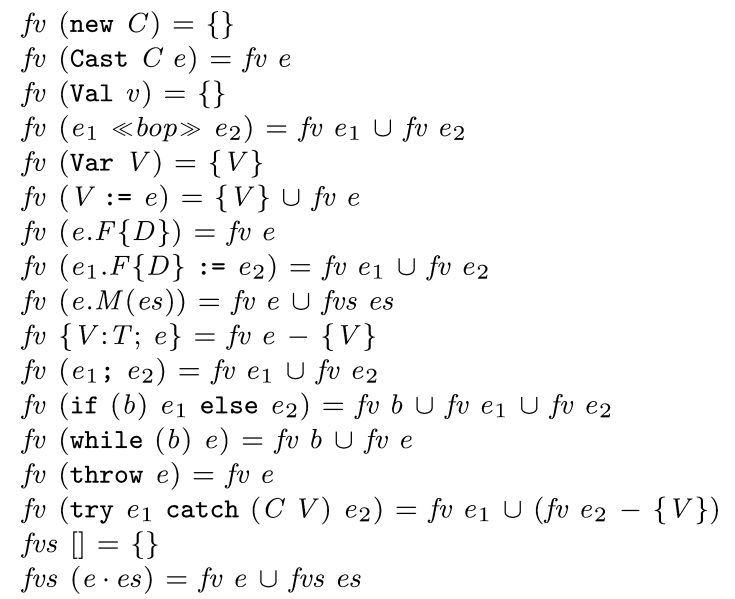

Fig. 11. Free variables.

variables of the body refer only to this and the parameter names. Formally,

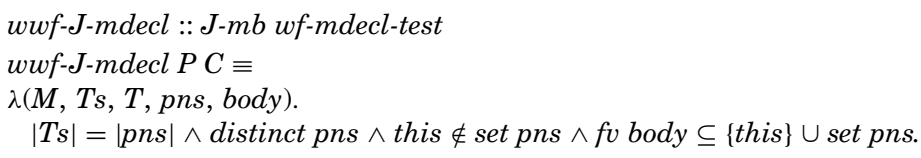

The key requirement is fv body $\subseteq\{$ this $\} \cup$ set pns; it rules out reference to global variables. This is necessary to make the big and small step semantics of method call coincide. In the big step semantics, body is evaluated in a store containing only this and the parameters. In the small step semantics, this and the parameters are (indirectly) added to the current store, which would lead to dynamic variable binding (see Section 2.3.2) if body contained free variables outside $\{$ this $\} \cup$ set pns.

The condition $|T s|=|p n s|$ is necessary because we have separated parameter names from their types. Normally, there is a combined parameter list of pairs $(V, T)$, just as in field declarations. However, since parameter names do not make sense on the machine level but parameter types do, we have separated these two concepts in our generic type of programs.

A Jinja program is weakly well-formed iff all its method bodies are:

$$
w w f-J-p r o g \equiv w f-p r o g w w f-J-m d e c l
$$

\subsection{Relating Big and Small Step Semantics}

Our big and small step semantics are equivalent in the following sense:

Theorem 2.3. If wwf-J-prog $P$, then

$P \vdash\langle e, s\rangle \Rightarrow\left\langle e^{\prime}, s^{\prime}\right\rangle$ iff $P \vdash\langle e, s\rangle \rightarrow^{*}\left\langle e^{\prime}, s\right\rangle \wedge$ final $e^{\prime}$.

One half of the only-if-direction of Theorem 2.3 is Lemma 2.1, and the other half is 
Theorem 2.4. If wwf-J-prog $P$ and $P \vdash\langle e, s\rangle \Rightarrow\left\langle e^{\prime}, s\right\rangle$ then $P \vdash\langle e, s\rangle \rightarrow^{*}$ $\left\langle e^{\prime}, s^{\prime}\right\rangle$.

Proof. The proof is by induction on $\Rightarrow$. Most cases follow the simple pattern that we demonstrate for Cast. First, we lift the subexpression reduction rules from $\rightarrow$ to $\rightarrow^{*}$, that is, we show $P \vdash\langle e, s\rangle \rightarrow^{*}\langle$ null, $s\rangle \Longrightarrow P \vdash\langle$ Cast $C e, s\rangle \rightarrow^{*}$ $\left\langle\right.$ null, $\left.s^{\dagger}\right\rangle$, which follows from rule $P \vdash\langle$ Cast $C$ null,s $\rangle \rightarrow\langle$ null,s $\rangle$ with the help of the lemma $P \vdash\langle e, s\rangle \rightarrow^{*}\left\langle e^{\prime}, s\right\rangle \Longrightarrow P \vdash\langle$ Cast $C e, s\rangle \rightarrow^{*}\left\langle\right.$ Cast $\left.C e^{\prime}, s\right\rangle$ which is proved from rule $P \vdash\langle e, s\rangle \rightarrow\left\langle e^{\prime}, s\right\rangle \Longrightarrow P \vdash\langle$ Cast $C e, s\rangle \rightarrow\left\langle\right.$ Cast $\left.C e^{\prime}, s\right\rangle$ by induction on $\rightarrow^{*}$. Now, the proposition follows by induction hypothesis.

For blocks (and similarly, for try-catch), the lifting is more complicated:

$\llbracket P \vdash\left\langle e_{0},\left(h_{0}, l_{0}(V:=\right.\right.$ None $\left.\left.)\right)\right\rangle \rightarrow^{*}\left\langle e_{2},\left(h_{2}, l_{2}\right)\right\rangle ;$ final $e_{2} \rrbracket$

$\Longrightarrow P \vdash\left\langle\left\{V: T ; e_{0}\right\},\left(h_{0}, l_{0}\right)\right\rangle \rightarrow^{*}\left\langle e_{2},\left(h_{2}, l_{2}\left(V:=l_{0} V\right)\right)\right\rangle$

is proved by induction on $\rightarrow^{*}$ in the premise. The induction step can be proved via

$\llbracket P \vdash\langle e,(h, l(V \mapsto v))\rangle \rightarrow^{*}\left\langle e^{\prime},\left(h^{\prime}, l^{\prime}\right)\right\rangle ;$ final $e^{\prime} \rrbracket$

$\Longrightarrow P \vdash\langle\{V: T ; V:=\operatorname{Val} v ; e\},(h, l)\rangle \rightarrow^{*}\left\langle e^{\prime},\left(h^{\prime}, l^{\prime}(V:=l V)\right)\right\rangle$

which follows easily from

$P \vdash\langle e,(h, l(V \mapsto v))\rangle \rightarrow^{*}\left\langle e^{\prime},\left(h^{\prime}, l^{\prime}\right)\right\rangle \Longrightarrow$

$P \vdash\langle\{V: T ; V:=\operatorname{Val} v ; e\},(h, l)\rangle \rightarrow^{*}\left\langle\left\{V: T ; V:=\operatorname{Val}\left(\right.\right.\right.$ the $\left.\left.\left.\left(l^{\prime} V\right)\right) ; e^{\prime}\right\},\left(h^{\prime}, l^{\prime}(V:=l V)\right)\right\rangle$,

which can be proved by induction on $\rightarrow^{*}$ in the premise.

The most complex case is a method call where we have to prove the small step simulation of the exception-free big step call rule, that is,

$\llbracket w w f-J-p r o g P ; P \vdash\left\langle e, s_{0}\right\rangle \rightarrow^{*}\left\langle a d d r a, s_{1}\right\rangle ; P \vdash\left\langle e s, s_{1}\right\rangle[\rightarrow]^{*}\left\langle\right.$ map $\left.\operatorname{Val} v s,\left(h_{2}, l_{2}\right)\right\rangle ;$

$h_{2} a=\lfloor(C, f s)\rfloor ; P \vdash C$ sees $M: T s \rightarrow T=($ pns, body $)$ in $D ;|v s|=|p n s|$;

$l_{2}{ }^{\prime}=[$ this $\mapsto$ Addr a, pns $[\mapsto] v s] ; P \vdash\left\langle\right.$ body, $\left.\left(h_{2}, l_{2}\right)\right\rangle \rightarrow^{*}\left\langle e f,\left(h_{3}, l_{3}\right)\right\rangle$; final ef

$\Longrightarrow P \vdash\left\langle e . M(e s), s_{0}\right\rangle \rightarrow^{*}\left\langle e f,\left(h_{3}, l_{2}\right)\right\rangle \rrbracket$.

It is straightforward to derive $P \vdash\left\langle e \cdot M(e s), s_{0}\right\rangle \rightarrow^{*}\left\langle\left(\right.\right.$ addra $\left.a \cdot M(e s), s_{1}\right\rangle \rightarrow^{*}$ $\left\langle(a d d r a) . M\left(\right.\right.$ map Val pvs), $\left.\left(h_{2}, l_{2}\right)\right\rangle \rightarrow\left\langle b l k s,\left(h_{2}, l_{2}\right)\right\rangle$ (where blks abbreviates blocks (this.pns, Class D.Ts, Addr a.pvs, body)) from the assumptions. From $P$ $\vdash\left\langle\operatorname{bod} y,\left(h_{2}, l_{2}\right)\right\rangle \rightarrow^{*}\left\langle e f,\left(h_{3}, l_{3}\right)\right\rangle(1)$ it follows by the easy lemma

$P \vdash\langle e,(h, l)\rangle \rightarrow^{*}\left\langle e^{\prime},\left(h^{\prime}, l^{\prime}\right)\right\rangle \Longrightarrow P \vdash\left\langle e,\left(h, l_{0}++l\right)\right\rangle \rightarrow^{*}\left\langle e^{\prime},\left(h^{\prime}, l_{0}++l^{\prime}\right)\right\rangle$

(provable by induction) that $P \vdash\left\langle\right.$ body, $\left(h_{2}, l_{2}(\right.$ this $\mapsto$ Addr $a$, pns $\left.\left.[\mapsto] p v s)\right)\right\rangle \rightarrow^{*}$ $\left\langle e f,\left(h_{3}, l_{2}++l_{3}\right)\right\rangle$. Now, we can transfer the bindings for this and pns from the store into blocks to obtain

$P \vdash\left\langle\right.$ blks,$\left.\left(h_{2}, l_{2}\right)\right\rangle \rightarrow^{*}\left\langle e f,\left(h_{3},\left(l_{2}++l_{3}\right)\left(l_{2} \mid\{\right.\right.\right.$ this $\} \cup$ set pns $\left.\left.)\right)\right\rangle$,

where $f(g \mid A)$ means $\lambda a$. if $a \in A$ then $g$ a else $f a$. Finally, we prove $\left(l_{2}++\right.$ $\left.l_{3}\right)\left(l_{2} \mid\{t h i s\} \cup\right.$ set pns $)=l_{2}(2)$, which finishes the call case. The proof of (2) is easy once we know dom $l_{3} \subseteq\{$ this $\} \cup$ set pns, which in turn follows from (1) using the lemma

$\llbracket w w f-J-p r o g P ; P \vdash\langle e,(h, l)\rangle \rightarrow^{*}\left\langle e^{\prime},\left(h^{\prime}, l^{\prime}\right)\right\rangle \rrbracket \Longrightarrow \operatorname{dom} l^{\prime} \subseteq \operatorname{dom} l \cup f v e$,

which in its turn is proved using the lemma

$\llbracket w w f-J-p r o g P ; P \vdash\langle e,(h, l)\rangle \rightarrow\left\langle e^{\prime},\left(h^{\prime}, l^{\prime}\right)\right\rangle \rrbracket \Longrightarrow f v e^{\prime} \subseteq f v e$, 
The other direction of Theorem 2.3

Theorem 2.5. If wwf-J-prog $P$ and $P \vdash\langle e, s\rangle \rightarrow^{*}\left\langle e^{\prime}, s^{\prime}\right\rangle$ and final $e^{\prime}$, then $P \vdash\langle e, s\rangle \Rightarrow\left\langle e^{\prime}, s^{\prime}\right\rangle$.

is proved easily by induction on $\rightarrow^{*}$ : the base case is Lemma 2.2 , the induction step follows directly from

Theorem 2.6. If wwf-J-prog $\mathrm{P}$ and $P \vdash\langle e, s\rangle \rightarrow\left\langle e^{\prime \prime}, s^{\prime \prime}\right\rangle$ and $P \vdash\left\langle e^{\prime \prime}, s^{\prime}\right\rangle \Rightarrow$ $\left\langle e^{\prime}, s\right\rangle$, then $P \vdash\langle e, s\rangle \Rightarrow\left\langle e^{\prime}, s\right\rangle$.

It is proved by induction on $\rightarrow$. Most cases are straightforward, except for method call which requires the following three lemmas:

$\llbracket|p s|=|T s| ;|p s|=|v s| ; P \vdash\langle$ blocks $(p s, T s, v s, e),(h, l)\rangle \Rightarrow\left\langle e^{\prime},\left(h^{\prime}, l^{\prime}\right)\right\rangle \rrbracket$

$\Longrightarrow \exists l^{\prime \prime} . P \vdash\langle e,(h, l(p s[\mapsto] v s))\rangle \Rightarrow\left\langle e^{\prime},\left(h^{\prime}, l^{\prime \prime}\right)\right\rangle$

$\llbracket w w f-J-p r o g P ; P \vdash\langle e,(h, l)\rangle \Rightarrow\left\langle e^{\prime},\left(h^{\prime}, l\right)\right\rangle ; f v e \subseteq W \rrbracket \Longrightarrow P \vdash\left\langle e,(h, l\lceil W)\rangle \Rightarrow\left\langle e^{\prime},\left(h^{\prime}, l^{\prime}\lceil W)\right\rangle\right.\right.$,

$\llbracket p\langle e,(h, l)\rangle \Rightarrow\left\langle e^{\prime},\left(h^{\prime}, l^{\prime}\right)\right\rangle ; f v e=\{\} \rrbracket \Longrightarrow l^{\prime}=l$.

The notation $m \uparrow_{\wedge A}$ means restriction of $m$ to $A$, that is, $\lambda x$. if $x \in A$ then $m x$ else None. The proofs of these lemmas and the proof of the method call case derived from them are reasonably straightforward.

Note that the fact that Theorem 2.6 holds is not just a nice coincidence; this theorem is trivially implied whenever $\Rightarrow$ and $\rightarrow^{*}$ coincide.

\subsection{Type System}

Having concluded the dynamic semantics, we now turn to context conditions, starting with the type system. Jinja types are either primitive (Boolean and Integer), class types Class $C, N T$ (the type of Null), or Void (the type of Unit). The corresponding HOL type is called ty. A reference type is either Class $C$ or $N T$-predicate is-refT :: ty $\Rightarrow$ bool tests for reference types.

Types should only refer to classes that exist in the current program:

is-type $P T \equiv$ case $T$ of Class $C \Rightarrow$ is-class $P C \mid-\Rightarrow$ True

Function typeof :: heap $\Rightarrow v a l \Rightarrow$ ty option computes the type of a value. The heap argument is necessary because values may contain addresses. The result type is ty option rather than ty because unallocated addresses do not have a type.

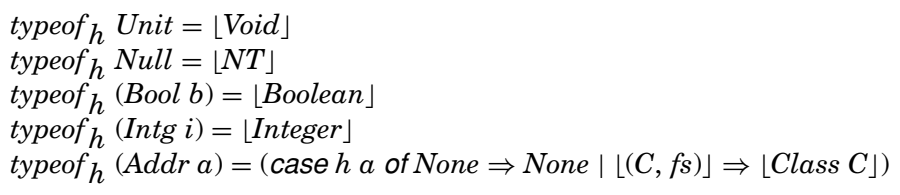

If we want to rule out addresses in values, we simply supply an empty heap and define the abbreviation

$$
\text { typeof } v \equiv \text { typeof }_{\text {empty }} v .
$$




$$
\begin{aligned}
& \frac{\text { is-class } P C}{P, E \vdash \text { new } C:: \text { Class } C} \quad \frac{\text { typeof } v=\lfloor T\rfloor}{P, E \vdash \operatorname{Val} v:: T} \quad \frac{E V=\lfloor T\rfloor}{P, E \vdash \operatorname{Var} V:: T} \\
& \frac{P, E \vdash e:: \text { Class } D \quad \text { is-class } P C \quad P \vdash C \preceq^{*} D \vee P \vdash D \preceq{ }^{*} C}{P, E \vdash \text { Cast } C \text { e :: Class } C} \\
& \frac{P, E \vdash e_{1}:: T_{1} \quad P, E \vdash e_{2}:: T_{2} \quad P \vdash T_{1} \leq T_{2} \vee P \vdash T_{2} \leq T_{1}}{P, E \vdash e_{1} \ll=\gg e_{2}:: \text { Boolean }} \\
& \frac{P, E \vdash e_{1}:: \text { Integer } \quad P, E \vdash e_{2}:: \text { Integer }}{P, E \vdash e_{1} \ll+e_{2}:: \text { Integer }} \\
& \frac{E V=\lfloor T\rfloor \quad P, E \vdash e:: T^{\prime} \quad P \vdash T^{\prime} \leq T \quad V \neq \text { this }}{P, E \vdash V:=e:: \text { Void }} \\
& \frac{P, E \vdash e:: \text { Class } C \quad P \vdash C \text { sees } F: T \text { in } D}{P, E \vdash e . F\{D\}:: T} \\
& \frac{P, E \vdash e_{1}:: \text { Class } C \quad P \vdash C \text { sees } F: T \text { in } D \quad P, E \vdash e_{2}:: T^{\prime} \quad P \vdash T^{\prime} \leq T}{P, E \vdash e_{1} . F\{D\}:=e_{2}:: \text { Void }} \\
& P, E \vdash e:: \text { Class } C \\
& \frac{P \vdash C \text { sees } M: T s \rightarrow T=(\text { pns, body }) \text { in } D \quad P, E \vdash \text { es }\left[:: T^{\prime} \quad P \vdash T s^{\prime}[\leq] T s\right.}{P, E \vdash e \cdot M(e s):: T} \\
& \frac{\text { is-type } P T \quad P, E(V \mapsto T) \vdash e:: T^{\prime}}{P, E \vdash\{V: T ; e\}:: T^{\prime}} \quad \frac{P, E \vdash e_{1}:: T_{1} \quad P, E \vdash e_{2}:: T_{2}}{P, E \vdash e_{1} ; e_{2}:: T_{2}} \\
& P, E \vdash e:: \text { Boolean } \\
& P, E \vdash e_{1}:: T_{1} \quad P, E \vdash e_{2}:: T_{2} \quad P \vdash T_{1} \leq T_{2} \vee P \vdash T_{2} \leq T_{1} \\
& \begin{array}{c}
P \vdash T_{1} \leq T_{2} \longrightarrow T=T_{2} \quad P \vdash T_{2} \leq T_{1} \longrightarrow T=T_{1} \\
P, E \vdash \text { if }(e) e_{1} \text { else } e_{2}:: T
\end{array} \\
& \frac{P, E \vdash e:: \text { Boolean } \quad P, E \vdash c:: T}{P, E \vdash \text { while }(e) c:: \text { Void }} \quad \frac{P, E \vdash e:: \text { Class } C}{P, E \vdash \text { throw } e:: \text { Void }} \\
& \frac{P, E \vdash e_{1}:: T \quad P, E(V \mapsto \text { Class } C) \vdash e_{2}:: T \quad \text { is-class } P C}{P, E \vdash \operatorname{try} e_{1} \text { catch }(C V) e_{2}:: T} \\
& P, E \vdash[][::][] \quad \frac{P, E \vdash e:: T \quad P, E \vdash e s[\because:] T s}{P, E \vdash e \cdot e s[:: T \cdot T s}
\end{aligned}
$$

Fig. 12. Typing rules.

The subclass relation $P \vdash C \preceq^{*} C^{\prime}$ induces a subtype relation $P \vdash T \leq T^{\prime}$ (often called widening) in the obvious manner:

$$
P \vdash T \leq T \quad P \vdash N T \leq \text { Class } C
$$

$$
\frac{P \vdash C \preceq^{*} D}{P \vdash \text { Class } C \leq \text { Class } D} .
$$

The pointwise extension of $\leq$ to lists of types is written $[\leq]$.

The core of the type system is the judgement $P, E \vdash e:: T$, where $E$ is an environment, that is, a map from variables to their types. The complete set of typing rules is shown in Figure 12. We only discuss the more interesting ones, starting with field access and field assignment. Their typing rules do not just enforce that the types fit together, but also that the annotation $\{D\}$ is correct: $\{D\}$ must be the defining class of the field $F$ visible from the static class of the object. 
Now we examine the remaining rules for $P, E \vdash e:: T$. We only allow up and down casts: other casts are pointless because they are bound to fail at runtime. The rules for $e_{1} \ll e_{2}$ and if $(e) e_{1}$ else $e_{2}$ follow Java (with its conditional operator ? : rather than its if-else) in requiring that the type of $e_{1}$ is a subtype of that of $e_{2}$ or conversely. Loops are of type Void because they evaluate to unit. Exceptions (throw) are of type Void, as in Java. They could also be polymorphic, but that would complicate the type system. The rule for try $e_{1}$ catch $(C V) e_{2}$ follows Java (where $e_{1}$ and $e_{2}$ must be statements) in requiring that $e_{1}$ and $e_{2}$ have the same type.

The extension of :: to lists is denoted by [::].

Although the rules for $P, E \vdash e:: T$ can be viewed as computing the annotations $\{D\}$ (via the constraint on $D$ ), an explicit computation $P, E \vdash e \sim e^{\prime}$ may be more clear: The input $e$ is an unannotated expression, and the output $e^{\prime}$ its annotated version. Here are two representative rules, one that just copies, and one that adds an annotation:

$$
\begin{gathered}
\frac{P, E \vdash e \leadsto e^{\prime}}{P, E \vdash \text { Cast } C e \leadsto \text { Cast } C e^{\prime}} \\
\frac{P, E \vdash e \leadsto e^{\prime} \quad P, E \vdash e^{\prime}:: \text { Class } C \quad P \vdash C \text { sees } F: T \text { in } D}{P, E \vdash e . F \leadsto e^{\prime} . F\{D\}}
\end{gathered}
$$

We also determine if some Var $V$ really refers to a variable or to a field. In the latter case, it is prefixed by this and annotated:

$$
\frac{E V=\lfloor T\rfloor}{P, E \vdash \operatorname{Var} V \leadsto \operatorname{Var} V} \quad \frac{E V=\text { None } \quad E \text { this }=\lfloor\text { Class } C\rfloor \quad P \vdash C \operatorname{sees} V: T \text { in } D}{P, E \vdash \operatorname{Var} V \leadsto \operatorname{Var} \text { this. } V\{D\}} .
$$

Thus $\leadsto$ should be viewed as a translation that is part of type checking. As we do not refer to it again, we need not show the remaining (obvious) rules.

\subsection{Definite Assignment}

One of Java's notable features is the check that all variables must be assigned to before use, called "definite assignment." Jinja's rules for definite assignment are much simpler than Java's, thus missing certain cases, but nonetheless demonstrating the feature in its full generality. We employ two recursive functions

$$
\mathcal{D}:: \text { expr } \Rightarrow \text { vname set option } \Rightarrow \text { bool } \quad \mathcal{A}:: \text { expr } \Rightarrow \text { vname set option. }
$$

For a moment, ignore the option type. Then, $\mathcal{D} e A$ is meant to check if evaluation of $e$ starting from any state where all variables in $A$ are initialized only accesses initialized variables. The auxiliary function $\mathcal{A} e$ computes the set of variables that have been assigned after any normal evaluation of $e$.

The need for option arises because of throw: What should $\mathcal{A}$ (throw $e$ ) return? We use None to indicate that the expression will always fail. However, we should already think of set option as a completion of set with a top element None representing the universal set of all variable names (called UNIV). We will discuss this issue again in the following, but first we look at the definition of $\mathcal{D}$ (Figure 13) and $\mathcal{A}$ (Figure 14). They use the auxiliary operations $\sqcup, \sqcap, \ominus$, $\sqsubseteq$, and $\in \in$ ( Figure 15) which extend $\cup, \cap,-$, and $\subseteq$ from set to set option, treating None (almost) as the universal set. 


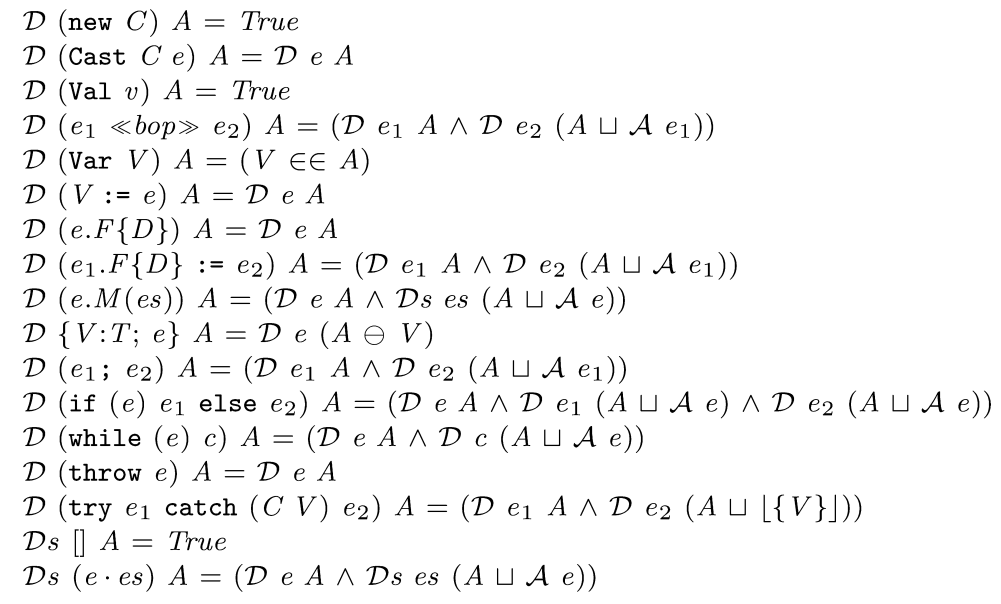

Fig. 13. Definition of $\mathcal{D}$.

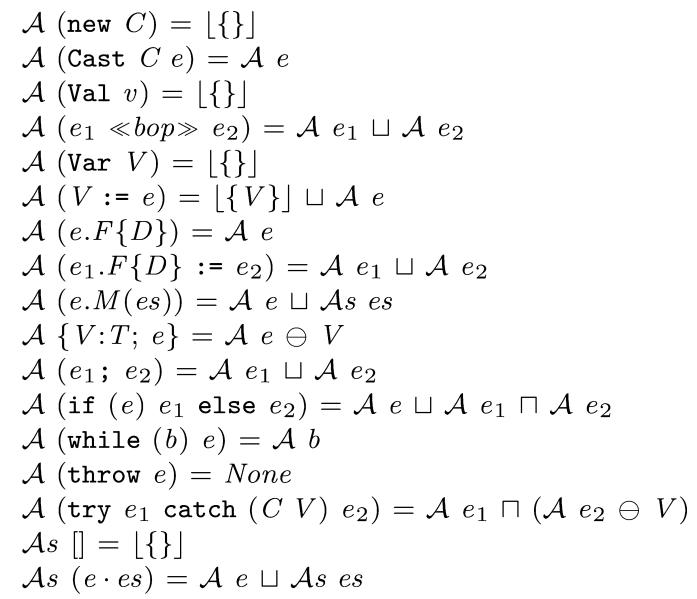

Fig. 14. Definition of $\mathcal{A}$.

$A \sqcup B \equiv$ case $A$ of None $\Rightarrow$ None $\mid\lfloor A\rfloor \Rightarrow$ case $B$ of None $\Rightarrow$ None $\mid\lfloor B\rfloor \Rightarrow\lfloor A \cup B\rfloor$

$A \sqcap B \equiv$ case $A$ of None $\Rightarrow B \mid\lfloor A\rfloor \Rightarrow$ case $B$ of None $\Rightarrow\lfloor A\rfloor \mid\lfloor B\rfloor \Rightarrow\lfloor A \cap B\rfloor$

$A \ominus a \equiv$ case $A$ of None $\Rightarrow$ None $\mid\lfloor A\rfloor \Rightarrow\lfloor A-\{a\}\rfloor$

$A \sqsubseteq B \equiv$ case $B$ of None $\Rightarrow$ True $\mid\lfloor B\rfloor \Rightarrow$ case $A$ of None $\Rightarrow$ False $\mid\lfloor A\rfloor \Rightarrow A \subseteq B$

$a \in \in A \equiv$ case $A$ of None $\Rightarrow$ True $\mid\lfloor A\rfloor \Rightarrow a \in A$

Fig. 15. Operations on set option . 
The rules for $\mathcal{D}$ and $\mathcal{A}$ are essentially straightforward and we just discuss a few of those defining $\mathcal{A}$ : Evaluating if $(e) e_{1}$ else $e_{2}$ guarantees all assignments that $e$ guarantees, and those that both $e_{1}$ and $e_{2}$ guarantee; evaluating while (b) $e$ guarantees only the assignments in $b$ because $e$ may never be evaluated; evaluating $\{V: T ; e\}$ cannot assign to $V$, because its $V$ is local. The test $\mathcal{D}$ e $A$ is even more uniform. It descends into all subexpressions of $e$ in the order in which they are evaluated, extends $A$ according to $\mathcal{A}$, and every time it comes across some $\operatorname{Var} V$, it checks if $V \in \in A$, that is, if $V$ has definitely been assigned to beforehand.

Monotonicity of $\mathcal{D}$ is proved by induction on $e$ :

Lemma 2.7. If $\mathcal{D}$ e $\mathrm{A}$ and $A \sqsubseteq A^{\prime}$ then $\mathcal{D}$ e $\mathrm{A}^{\prime}$.

Let us now return to the question of None vs. UNIV. If we worked with set as opposed to set option, we would need to define $\mathcal{A}$ (throw $)=U N I V$ to ensure that $\mathcal{A}$ (if (e) throw_else $\left.e_{2}\right)=\mathcal{A} e \cup \mathcal{A} e_{2}$, that is, that $\mathcal{A}$ (throw _) does not reduce the overall result, which should only reflect normal evaluations. Returning UNIV in the case of guaranteed throw is what Schirmer [2003] chooses. For a start, this loses direct executability, as $U N I V$ is not a finite set. What is worse, in Jinja it would lead to undesirable imprecision of the analysis. Let $e$ be the expression if $(\operatorname{Var} B)\{V: T$; throw $\}$ else $V:=$ true. We would obtain $\mathcal{A}\{V: T$; throw $\}=$ UNIV $-\{V\}, \mathcal{A} e=\{\}$, and hence, $\neg \mathcal{D}(e ; V:=\operatorname{Var} V)\{B\}$, contrary to what we would expect. This is where None comes in: We would like $U N I V-\{V\}=$ UNIV, but since this does not hold, we introduce None as the new top element of the lattice and define $\ominus$ such that None $\ominus V=$ None. Schirmer [2003] gets away with UNIV because he does not allow the aforementioned $e$; it contains both a local and a global $V$, something that Java forbids. In Jinja, however, we do not want to ban nested declarations of the same variable, which cripples the classic block structure.

\subsection{Well-Formed Jinja Programs}

Well-formedness of Jinja method declarations

$w f-J-m d e c l P C(M, T s, T, p n s, b o d y) \equiv$

$|T s|=\mid$ pns $\mid \wedge$ distinct pns $\wedge$ this $\notin$ set pns $\wedge$

$\left(\exists T^{\prime} . P,[\right.$ this $\mapsto$ Class $C$, pns $[\mapsto] T s] \vdash$ body $\left.:: T^{\prime} \wedge P \vdash T^{\prime} \leq T\right) \wedge$

$\mathcal{D}$ body $\lfloor\{$ this $\} \cup$ set pns $\rfloor$

extends weak well-formedness (Section 2.4.1) by requiring that method bodies: (a) are well-typed with a subtype of the declared return type, and (b) pass the definite assignment test assuming only this and the parameters are initialized. A Jinja program is well-formed iff all its method bodies are

$$
w f-J-p r o g \equiv w f-p r o g w f-J-m d e c l
$$

\subsection{Type Safety}

In Section 2.9 we prove type safety in the traditional syntactic way [Wright and Felleisen 1994]: we show progress (every well-typed expression that is not final can reduce) and subject reduction (well-typed expressions reduce to well-typed 
expressions and their type may only become more specific). These inductive proofs need a number of new notions (invariants) to go through.

2.9.1 Conformance. This expresses that semantic objects conform to their syntactic description. Let $v_{m}$ be a map to values and $T_{m}$ be a map to types:

Conformance of values to types.

$P, h \vdash v: \leq T \equiv \exists T^{\prime}$. typeof $_{h} v=\left\lfloor T^{\prime}\right\rfloor \wedge P \vdash T^{\prime} \leq T$

Conformance of fields to types.

$P, h \vdash v_{m}(: \leq) T_{m} \equiv \forall F D T . T_{m} F D=\lfloor T\rfloor \longrightarrow\left(\exists v \cdot v_{m} F D=\lfloor v\rfloor \wedge P, h \vdash v: \leq T\right)$

Weak conformance of local variables to types.

$P, h \vdash v_{m}(: \leq)_{w} T_{m} \equiv \forall V v \cdot v_{m} V=\lfloor v\rfloor \longrightarrow\left(\exists T . T_{m} V=\lfloor T\rfloor \wedge P, h \vdash v: \leq T\right)$

Conformance of objects.

$P, h \vdash o b j \sqrt{ } \equiv$

let $\left(C, v_{m}\right)=$ obj in $\exists$ FDTs. $P \vdash C$ has-fields FDTs $\wedge P, h \vdash v_{m}(: \leq)$ map-of FDTs

Conformance of heaps.

$P \vdash h \sqrt{ } \equiv(\forall a$ obj. $h a=\lfloor o b j\rfloor \longrightarrow P, h \vdash o b j \sqrt{ }) \wedge$ preallocated $h$

preallocated $h \equiv \forall C \in$ sys-xcpts. $\exists f$ s. $h$ (addr-of-sys-xcpt $C)=\lfloor(C, f s)\rfloor$

Conformance of states.

$P, E \vdash s \sqrt{ } \equiv$ let $(h, l)=s$ in $P \vdash h \sqrt{ } \wedge P, h \vdash l(: \leq)_{w} E$

Note that $(: \leq)$ says that all declared fields must have values of the right type, whereas $(: \leq)_{w}$ says that only initialized variables must have values of the right type. This reflects the difference in initialization of fields and local variables.

2.9.2 Runtime Type System. The proof of subject reduction requires a modified type system. The purpose of $\vdash::$ ( Figure 12) is to rule out not just unsafe expressions, but ill-formed ones in general. For example, assignments to this are considered bad style and are thus ruled out, although such assignments are perfectly safe (and are in fact allowed in the JVM). But now we need a type system that is just strong enough to characterize absence of type safety violations and is invariant under reduction. For a start, during reduction, expressions containing addresses may arise. To make them well-typed, the runtime type system [Drossopoulou and Eisenbach 1999] also takes the heap into account (to look up the class of an object) and is written $P, E, h \vdash e: T$. But there are more subtle changes exemplified by the rule for field access: $P, E \vdash e . F\{D\}:: T$ requires $P \vdash C$ sees $F: T$ in $D$ if $e$ is of class $C$. If $e$ reduces to an object belonging to a subclass of $C$, this condition may no longer be met. Thus, we relax this to $P \vdash C$ has $F: T$ in $D$ which is preserved by reduction and is still strong enough to imply type safety. It is interesting to note that this change was missed by Flatt et al. [1999], which invalidates their Lemma 6 and thus subject reduction. Please keep in mind that the runtime type system is a purely technical device needed in the proof of type safety.

ACM Transactions on Programming Languages and Systems, Vol. 28, No. 4, July 2006. 


$$
\begin{aligned}
& \frac{\text { typeof }_{h} v=\lfloor T\rfloor}{P, E, h \vdash \text { Val } v: T} \quad \frac{P, E, h \vdash e: T \quad \text { is-refT } T \quad \text { is-class } P C}{P, E, h \vdash \text { Cast } C e: \text { Class } C} \\
& \frac{P, E, h \vdash e_{1}: T_{1} \quad P, E, h \vdash e_{2}: T_{2}}{P, E, h \vdash e_{1} \ll e_{2}: \text { Boolean }} \quad \frac{E V=\lfloor T\rfloor \quad P, E, h \vdash e: T^{\prime} \quad P \vdash T^{\prime} \leq T}{P, E, h \vdash V:=e: \text { Void }} \\
& \frac{P, E, h \vdash e: \text { Class } C \quad P \vdash C \text { has } F: T \text { in } D}{P, E, h \vdash e . F\{D\}: T} \quad \frac{P, E, h \vdash e: N T}{P, E, h \vdash e . F\{D\}: T} \\
& \frac{P, E, h \vdash e_{1}: \text { Class } C \quad P \vdash C \text { has } F: T \text { in } D \quad P, E, h \vdash e_{2}: T_{2} \quad P \vdash T_{2} \leq T}{P, E, h \vdash e_{1} . F\{D\}:=e_{2}: \text { Void }} \\
& \frac{P, E, h \vdash e_{1}: N T \quad P, E, h \vdash e_{2}: T_{2}}{P, E, h \vdash e_{1} \cdot F\{D\}:=e_{2}: \text { Void }} \quad \frac{P, E, h \vdash e: N T \quad P, E, h \vdash e s[:] T s}{P, E, h \vdash e \cdot M(e s): T} \\
& \frac{P, E(V \mapsto T), h \vdash e: T^{\prime}}{P, E, h \vdash\{V: T ; e\}: T^{\prime}} \quad \frac{P, E, h \vdash e: T_{r} \quad \text { is-refT } T_{r}}{P, E, h \vdash \text { throw } e: T} \\
& \frac{P, E, h \vdash e_{1}: T_{1} \quad P, E(V \mapsto \text { Class } C), h \vdash e_{2}: T_{2} \quad P \vdash T_{1} \leq T_{2}}{P, E, h \vdash \operatorname{try} e_{1} \text { catch }(C V) e_{2}: T_{2}}
\end{aligned}
$$

Fig. 16. Core of runtime typing rules.

In Figure 16 we show only those rules for $\vdash:$ (and $\vdash$ [:]) that differ from their ::-counterpart beyond the addition of $h$. The most frequent change is the following. Expressions that are required to be of class type by :: may reduce to null. In order to preserve well-typedness we have to add rules for the cases $e::$ $N T$ in $e . F\{D\}, e . F\{D\}:=e_{2}$, and e.M(es). Note that we lose uniqueness of typing: null. $F\{D\}$ unavoidably does not even have a unique least type anymore. A similar situation arises with throw $e$ and Cast $C e$, where we avoid an additional rule by requiring $e$ to be of reference type (which includes $N T$ ). For try $e_{1}$ catch $(C V) e_{2}$, we no longer require $e_{1}$ and $e_{2}$ to have the same type because reduction of $e_{1}$ may also have reduced its type. Then there is the change from sees to has for field access and update. And finally, we drop two preconditions in the rules for $V:=e$ and $\{V: T ; e\}$ just to show that they are orthogonal to type safety.

2.9.3 The Type Safety Proof. Under suitable conditions we can now show progress:

Lemma 2.8. (Progress) If wwf-J-prog $P$ and $P \vdash h \sqrt{ }$ and $P, E, h \vdash e: T$ and $\mathcal{D} e\lfloor$ dom $l\rfloor$ and $\neg$ final $e$, then $\exists e^{\prime} s^{\prime} . P \vdash\langle e,(h, l)\rangle \rightarrow\left\langle e^{\prime}, s^{\prime}\right\rangle$.

The proof is by induction on $P, E, h \vdash e: T$. Because of the special treatment of $\{V: T ; V:=\mathrm{Val} v ;$, $\}$, we need a slightly modified induction scheme with a separate rule for this case. Alternatively, we can do an induction on the size of $e$.

Let us examine the necessity for the individual premises. Weak wellformedness of $P$ is needed to ensure that each method declaration has as many parameter types as parameter names, a precondition of the method call rule. In addition, well-formedness is necessary for the following subtle reason: Even if $P$ defines a class $C$, relations has-fields (needed for the reduction of new) and sees (needed for the reduction of method calls) are only defined if $P$ is well-formed because acyclicity is needed in the traversal of the class hierarchy. 
Well-typedness of $e$ is needed, for example, to ensure that in every method call, the number of formal and actual parameters agrees. Heap conformance $(P \vdash$ $h \sqrt{ }$ ) is needed because otherwise, an object may not have all the fields of its class and field access may get stuck. Definite assignment is required to ensure that variable access does not get stuck.

Eventually, we show that a sequence of reductions preserves well-typedness by showing that each reduction preserves well-typedness. However, preservation of well-typedness requires additional assumptions, for example, conformance of the initial heap. Thus, we need to show conformance of all intermediate heaps, that is, preservation of heap conformance with each step. We need three auxiliary preservation theorems which are all proved by induction on $P \vdash\langle e,(h, l)\rangle \rightarrow\left\langle e^{\prime},\left(h^{\prime}, l^{\prime}\right)\right\rangle:$

Theorem 2.9. If $P \vdash\langle e,(h, l)\rangle \rightarrow\left\langle e^{\prime},\left(h^{\prime}, l^{\prime}\right)\right\rangle$ and $P, E, h \vdash e: T$ and $P \vdash h \sqrt{ }$ then $P \vdash h^{\prime} \sqrt{ }$.

Theorem 2.10. If $P \vdash\langle e,(h, l)\rangle \rightarrow\left\langle e^{\prime},\left(h^{\prime}, l^{\prime}\right)\right\rangle$ and $P, E, h \vdash e: T$ and $P, h \vdash$ $l(: \leq)_{w} E$, then $P, h^{\prime} \vdash l^{\prime}(: \leq)_{w} E$.

Theorem 2.11. If $w f$ - $J$-prog $P$ and $P \vdash\langle e,(h, l)\rangle \rightarrow\left\langle e^{\prime},\left(h^{\prime}, l^{\prime}\right)\right\rangle$ and $\mathcal{D} e$ $\lfloor$ dom $l\rfloor$, then $\mathcal{D} e^{\prime}\left\lfloor\right.$ dom $\left.l^{\prime}\right\rfloor$.

Because preservation of definite assignment has not been treated in the literature before, we look at a typical case in detail: sequential composition. We assume $\mathcal{D}\left(e ; e_{2}\right)\lfloor$ dom $l\rfloor$, that is, $\mathcal{D} e\lfloor$ dom $l\rfloor \wedge \mathcal{D} e_{2}(\lfloor$ dom $l\rfloor \sqcup \mathcal{A} e)$ and have to show $\mathcal{D}\left(e^{\prime} ; e_{2}\right)\left\lfloor\right.$ dom $\left.l^{\prime}\right\rfloor$, that is, $\mathcal{D} e^{\prime}\left\lfloor\right.$ dom $\left.l^{\prime}\right\rfloor \wedge \mathcal{D} e_{2}\left(\left\lfloor d o m l^{\prime}\right\rfloor \sqcup \mathcal{A} e^{\prime}\right)$. From $\mathcal{D} e\lfloor d o m ~ l\rfloor$ it follows by induction hypothesis that $\mathcal{D} e^{\prime}\left\lfloor d o m l^{\prime}\right\rfloor$, and from $\mathcal{D} e_{2}(\lfloor d o m l\rfloor \sqcup \mathcal{A} e)$ it follows with lemma

$P \vdash\langle e,(h, l)\rangle \rightarrow\left\langle e^{\prime},\left(h^{\prime}, l^{\prime}\right)\right\rangle \Longrightarrow\lfloor\operatorname{dom} l\rfloor \sqcup \mathcal{A} e \sqsubseteq\left\lfloor\operatorname{dom} l^{\prime}\right\rfloor \sqcup \mathcal{A} e^{\prime}$

(which is proved by induction over $\rightarrow$ ) together with monotonicity of $\mathcal{D}$ that $\mathcal{D} e_{2}\left(\left\lfloor\right.\right.$ dom $\left.\left.l^{\prime}\right\rfloor \sqcup \mathcal{A} e^{\prime}\right)$, thus concluding the case.

The main preservation theorem is single step subject reduction:

Theorem 2.12. If $w f-J-p r o g ~ P$ and $P \vdash\langle e, s\rangle \rightarrow\left\langle e^{\prime}, s\right\rangle$ and $P, E \vdash s \sqrt{ }$ and $P, E, h p s \vdash e: T$, then $\exists T^{\prime} . P, E, h p s^{\prime} \vdash e^{\prime}: T^{\prime} \wedge P \vdash T^{\prime} \leq T$.

The proof is again by induction on $\rightarrow$.

Now we extend subject reduction to $\rightarrow^{*}$. To ease notation we introduce the following definition

$P, E, s \vdash e: T \sqrt{ } \equiv P, E \vdash s \sqrt{ } \wedge P, E, h p s \vdash e: T$.

Now we can combine the auxiliary preservation theorems and subject reduction:

$\llbracket w f-J-p r o g P ; P \vdash\langle e, s\rangle \rightarrow\left\langle e^{\prime}, s^{\prime}\right\rangle ; P, E, s \vdash e: T \sqrt{ } \rrbracket$

$\Longrightarrow \exists T^{\prime} . P, E, s^{\prime} \vdash e^{\prime}: T^{\prime} \sqrt{ } \wedge P \vdash T^{\prime} \leq T$.

Induction yields the final form of subject reduction:

Theorem 2.13. If $w f-J-p r o g ~ P$ and $P \vdash\langle e, s\rangle \rightarrow^{*}\left\langle e^{\prime}, s\right\rangle$ and $P, E, s \vdash e: T \sqrt{ }$, then $\exists T^{\prime} . P, E, s^{\prime} \vdash e^{\prime}: T^{\prime} \sqrt{ } \wedge P \vdash T^{\prime} \leq T$.

ACM Transactions on Programming Languages and Systems, Vol. 28, No. 4, July 2006. 
Combining this theorem, the extension of Theorem 2.11 to $\rightarrow^{*}$, and progress, and replacing the runtime type system by the original one yields

Corollary 2.14. (Type Safety) if wf-J-prog $P$ and $P, E \vdash s \sqrt{ }$ and $P, E \vdash e:: T$ and $\mathcal{D} e\left\lfloor\right.$ dom (lcl s)」 and $P \vdash\langle e, s\rangle \rightarrow^{*}\left\langle e^{\prime}, s^{\prime}\right\rangle$ and $\nexists e^{\prime \prime} s^{\prime \prime} . P \vdash\left\langle e^{\prime}, s\right\rangle \rightarrow\left\langle e^{\prime \prime}, s^{\prime \prime}\right\rangle$ then $\left(\exists v \cdot e^{\prime}=\operatorname{Val} v \wedge P, h p s^{\prime} \vdash v: \leq T\right) \vee\left(\exists a \cdot e^{\prime}=\right.$ Throw $\left.a \wedge a \in \operatorname{dom}\left(h p s^{\prime}\right)\right)$.

If the program and the initial state are ok, and the expression is well-typed (w.r.t. $\vdash::$ ) and has the definite assignment property, then reduction to normal form either yields a value of a subtype of the initial expression or throws an existing object.

Note that we intentionally leave out the details of many of these proofs because type safety proofs abound in the literature.

\subsection{Related Work}

Most closely related to our work is that of Nipkow and Oheimb [1998, 1999], which provided the starting point for our big step semantics. Also closely related is the small step semantics by Drossopoulou and Eisenbach [1999]. The main difference is that they distinguish three very similar languages and transform from one into the next, whereas we have intentionally started with the "enriched language" (hence the class annotations in field references) and have taken care to identify it with the "runtime language." There are also a number of smaller differences (for example, we have omitted interfaces and arrays for space reasons, although they were present in our previous work [Nipkow and Oheimb 1998]), in particular, the way in which method calls are unfolded. We use local variables to hold the parameter values, whereas they choose new names and add those to the store. A name is new if it is not yet in the domain of the store. This scheme would need to be refined in a language like Jinja, where we can have uninitialised local variables. Also closely related is the small step semantics by Flatt et al. [1999] who define a much smaller subset of Java without mutable variables. Nobody seems to have connected these two styles of Java semantics before except Nipkow [2005], who obtained an even closer correspondence, but at the cost of allowing dynamic binding in the big step semantics, as well. Syme [1999] formalised the work by Drossopoulou and Eisenbach [1999] in his theorem prover DECLARE. The main difference is that his store is a list of maps, one for each method invocation. DECLARE, just like Isar [Wenzel 2002], aims at readable proofs. Ancona et al. [2001] analyse a feature of Java we have ignored, namely, the possibility of declaring which exceptions a method may raise. This leads to very subtle interactions between type system and exceptions. Schirmer [2004] has analysed the interaction of packages and access modifiers. On the other end of the spectrum, we have Featherweight Java [Igarashi et al. 2001], a minimal subset of Java which was used to study type soundness proofs. On the borderline of program verification, we have the denotational semantics of Java by Huisman [2001]. Further aspects formalised in the literature but beyond the scope of this article include multithreading, dynamic class loading, inner classes, generic classes, and mixins. 


\section{JINJA VIRTUAL MACHINE}

This section presents the machine model of the Jinja virtual machine (Section 3.1) and its operational semantics, first without (Section 3.2) and then with (Section 3.3) runtime type checks.

\subsection{Machine Model}

The model of the Jinja VM comprises the state space and the definition of method bodies. The state space of the Jinja VM is modelled closely after the Java VM. The state consists of a heap, a stack of call frames, and a flag signifying whether an exception was raised (and if yes, a reference to the exception object).

$$
\text { types jum-state }=\text { addr option } \times \text { heap } \times \text { frame list }
$$

The heap is the same as in the source language. The exception flag corresponds to the expression throw in the source language. The frame list is new.

Each method execution gets its own call frame containing its own operand stack (a list of values), its own set of registers ${ }^{2}$ for local variables (also a list of values), and its own program counter. We also store the class and name of the method and arrive at:

$$
\begin{aligned}
\text { types } & \text { frame }=\text { opstack } \times \text { registers } \times \text { cname } \times \text { mname } \times p c \\
\text { opstack } & =\text { val list } \\
\text { registers } & =\text { val list }
\end{aligned}
$$

It will turn out that the list of local variables is of fixed length, that is, it does not change with program execution. In fact, it is only modified and accessed by updating and indexing it at some position. Hence it can be implemented as an array. The size of the operand stack may change during execution, but here the maximum size is known statically. This enables efficient implementation for the stack, as well. Although the registers do not exclusively store the local variables of the method, but also its parameters and this-pointer, we use the terms registers and local variables interchangeably when the distinction is not important or clear from the context.

The instruction set of the Jinja VM is listed in Figure 17. The instructions are a bit more abstract than comparable Java VM instructions, but no further conceptual simplifications have been made. In Java, there is, for instance, a separate Load instruction for most of the basic machine types, while in Jinja there is only one polymorphic instruction. The more high-level instructions (that may seem to be a substantial simplification of a real machine such as Getfield, Putfield, and Invoke) have a direct correspondence to instructions in the Java VM.

Method bodies are lists of instructions together with an exception table and two numbers, $m x s$ and $m x l_{0}$. These latter are the maximum operand stack size and the number of local variables (not counting the this-pointer and the

${ }^{2}$ We deliberatly deviate here from the nomenclature of the JVM specification that calls these local variables. They hold the this-pointer, the parameters, and what would be the local variables in the source language. It will be important to distinguish between these in the discussion about the compiler to follow.

ACM Transactions on Programming Languages and Systems, Vol. 28, No. 4, July 2006. 

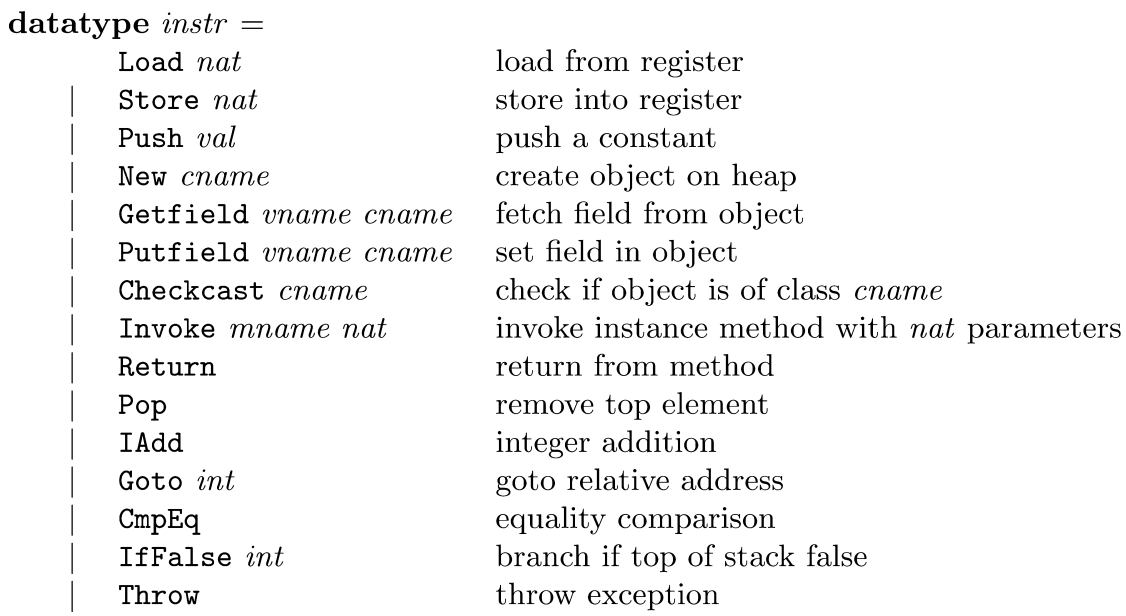

Fig. 17. The Jinja bytecode instruction set.

parameters of the method, which are stored in the first 0 to $n$ registers). So, the type parameter ' $m$ for method bodies gets instantiated with $n a t \times$ nat $\times$ instr list $\times$ ex-table:

$$
\begin{gathered}
\text { types } \quad \text { jum-method }=\text { nat } \times \text { nat } \times \text { instr list } \times \text { ex-table } \\
\text { jum-prog }=\text { jum-method prog }
\end{gathered}
$$

The exception table is a list of tuples $(f, t, C, h, d)$ :

$$
\text { types ex-table }=(\text { nat } \times \text { nat } \times \text { cname } \times \text { nat } \times \text { nat }) \text { list }
$$

The asymmetric interval $[f, t)$ denotes those instructions in the method body that correspond to the try block on the source level. The handler pc $h$ points to the first instruction of the corresponding catch block. The code starting at $h$ is the exception handler, and $d$ is the size of the stack the exception handler expects. An exception handler protects a program position $p c$ iff $p c \in[f, t)$. An exception table entry matches an exception $E$ if the handler protects the current $p c$ and if the class of $E$ is a subclass of $C$.

\subsection{Operational Semantics}

This section defines the state transition relation of the Jinja VM.

For easy direct executability, our main definition of the operational semantics of the Jinja VM is written in a functional rather than a relational style. The function exec :: jum-prog $\Rightarrow$ jum-state $\Rightarrow$ jvm-state option describes one-step execution:

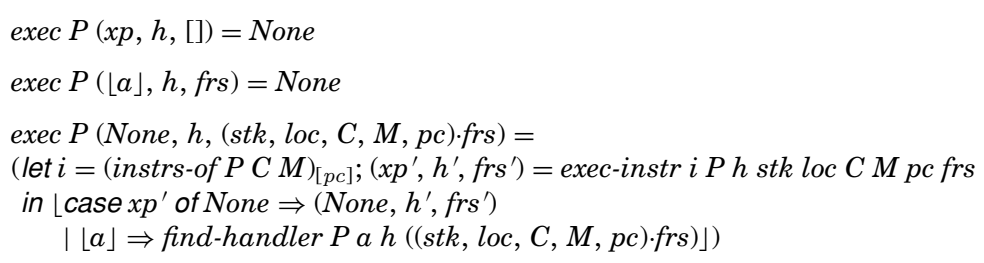


This says that execution halts if the call frame stack is empty or if an unhandled exception has occurred. In all other cases, execution is defined: Function exec decomposes the top call frame, retrieves the instruction list of the current method via instrs-of, delegates actual execution for single instructions to exec-instr, and finally sets the $p c$ to the appropriate exception handler (with find-handler) if an exception has occurred.

The function instrs-of selects the instruction sequence of method $M$ in class $C$ of program $P$. As the operational semantics of the Jinja VM at this level is phrased in a functional rather than a relational style, we turn the field and method accessor relations of Section 2.1.4 into functions. To look up methods, we use method, to look up fields, we use field. They satisfy:

$P \vdash C$ sees $M: T s \rightarrow T=m$ in $D \Longrightarrow \operatorname{method} P C M=(D, T s, T, m)$

$P \vdash C$ sees $F: T$ in $D \Longrightarrow$ field $P C F=(D, T)$.

Exception handling in find-handler (definition omitted) is similar to the Java VM: It looks up the exception table in the current method, and sets the program counter to the first handler that protects $p c$ and that matches the exception class. If there is no such handler, the topmost call frame is popped, and the search continues recursively in the invoking frame. If no exception handler is found, the exception flag remains set and the machine halts. If this procedure does find an exception handler $(f, t, C, h, d)$, it cuts down the operand stack of the frame to $d$ elements, puts a reference to the exception on top, and sets the $p c$ to $h$. This is different from the Java VM, where the stack is always emptied. Thus, exception handling in the Jinja VM is a generalisation of the Java VM where $d$ is always 0 . Leaving a number of elements on the stack gives us a nice way to translate the try-catch constructs of the source language that handle exceptions in the middle of an expression, as opposed to just on the statement level as in Java.

For some proofs the relational view is more convenient than the functional one. Therefore, we also define the one-step state transition relation:

$$
P \vdash \sigma \stackrel{\text { jvm }}{\longrightarrow} \sigma^{\prime}=\left(\text { exec } P \sigma=\left\lfloor\sigma^{\prime}\right\rfloor\right) .
$$

The state transition relation $\stackrel{\mathrm{jvm}}{\longrightarrow}$ for any finite number of steps is the reflexive transitive closure of $\stackrel{\text { jvm }}{\longrightarrow} 1$.

The definition of exec-instr in Figure 18 is large, but straightforward. The parameters of exec-instr are the following: the instruction to execute, the program $P$, the heap $h$, the operand stack stk and local variables loc of the current call frame, the class $C_{0}$ and name $M_{0}$ of the method that is currently executed, the current $p c$, and the rest of the call frame stack frs. One of the smaller definitions in exec-instr is the one for the IAdd instruction:

exec-instr IAdd $P h$ (Intg $i_{2} \cdot \operatorname{Intg} i_{1} \cdot$ stk) loc $C_{0} M_{0} p c$ frs $=$

(None, $\left.h,\left(\operatorname{Intg}\left(i_{1}+i_{2}\right) \cdot s t k, l o c, C_{0}, M_{0}, p c+1\right) \cdot f r s\right)$.

This takes the top two values as integers from the stack, adds them, and puts the result back onto the stack. The program counter is incremented, the rest remains untouched.

ACM Transactions on Programming Languages and Systems, Vol. 28, No. 4, July 2006. 


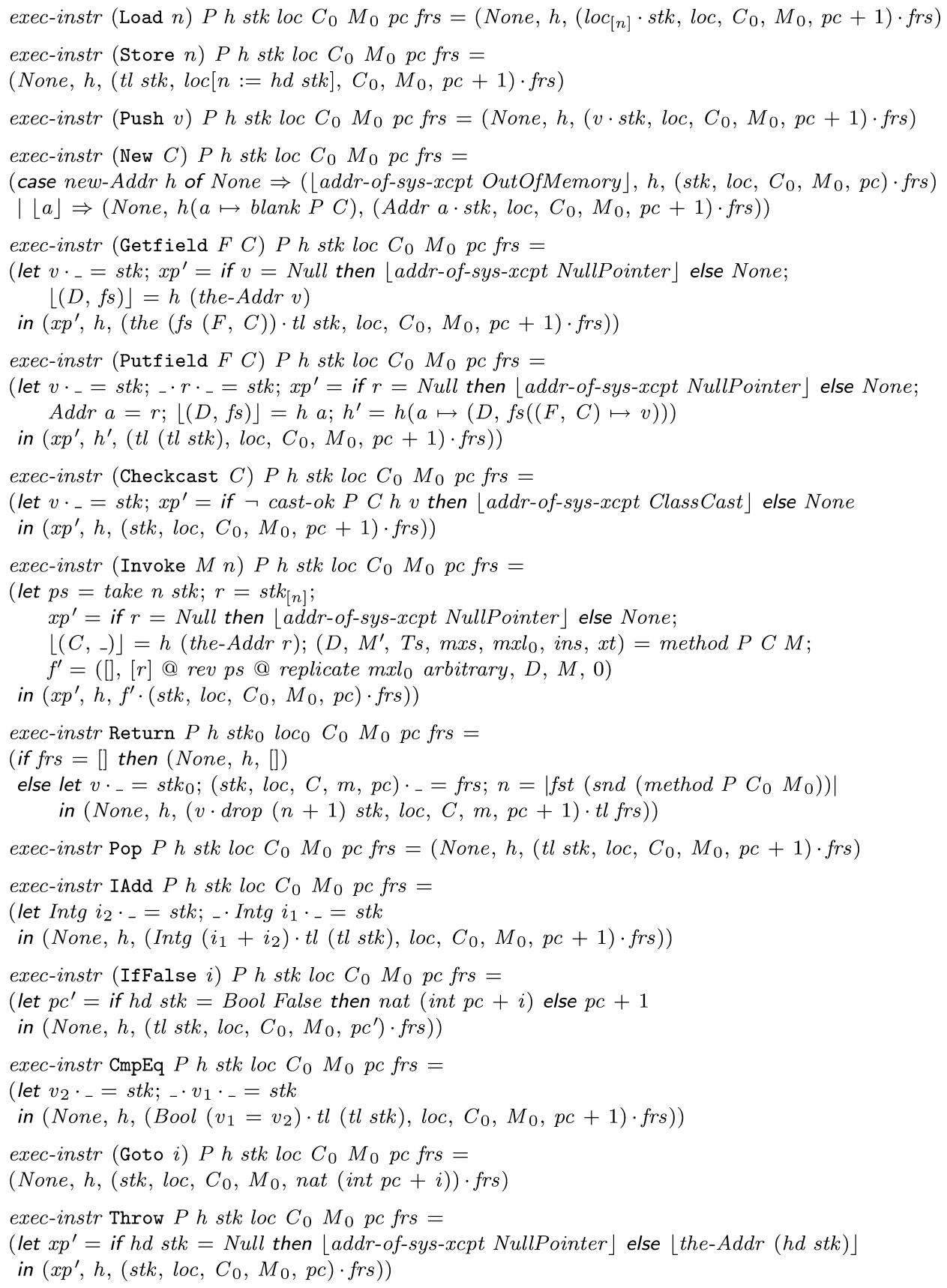

Fig. 18. Single-step execution. 
Most instructions in Figure 18 are of this simple form. Some of them use new functions: $h d$ and $t l$ return the head and tail of a list, and the destructor the-Addr is defined by the equality the-Addr $(A d d r a)=a$. The New instruction needs new-Addr, which was introduced in Section 2.2. A new object of class $C$ with all fields set to default values is produced by blank $P C$ (not shown). Remember that the field part of objects is a map from name and defining class to value, so $f s(F, C)$ used for Getfield and Putfield is the value of field $F$ defined in class $C$. The Checkcast instruction uses cast-ok $P C h v$ (also not shown) to check if the value $v$ is an address that points to an object of, at least, class $C$.

The definition for Invoke $M n$ is the most complex: It first uses take $n$ stk to get the the first $n$ elements of the stack (the parameters in reverse order), then it looks up the dynamic class $C$ of the object, determines the correct method (using method $P(C M$ ), and finally constructs the new state. If the object reference $r$ is $N u l l$, an exception is thrown, otherwise a new call frame for the invoked method is prepared. The new call frame has an empty operand stack, the object reference $r$ as the this-pointer in local variable 0, the parameters (rev ps is $p s$ in reverse order) in the next $n$ variables, and the rest of the local variables filled with a dummy value arbitrary (replicate $n v$ is a list of length $n$ that contains only $v$-elements). The new call frame also records the method (defining class $D$ and name $M$ ), and has the $p c$ set to 0 .

It is in this Invoke context that Return is best understood: If the current frame is the only one on the frame stack, the machine halts; if the current frame is not the only one, then the next frame on the stack must be the caller where the corresponding Invoke occurred. The Return instruction removes the current frame from the call stack and manipulates the caller frame. In the caller, it drops the parameters and the object reference, that is, $n+1$ elements, from the stack (drop $n x s$ is the dual to take $n x s$ ), puts the return value $v$ on top, and increments the $p c$.

This style of VM is also called aggressive because it does not perform any runtime type or sanity checks. It just assumes that everything is as expected, for example, for IAdd it assumes that there are indeed two integers on the stack. If the situation is not as expected, the operational semantics is unspecified at this point. In Isabelle, this means that there is a result (because HOL is a logic of total functions), but nothing is known about that result. It is the task of the bytecode verifier to ensure that this does not occur.

\subsection{A Defensive VM}

Although it is possible to prove type safety by using the aggressive VM alone, it is crisper to write and more obvious to see what the bytecode verifier guarantees when we additionally look at a defensive VM. The defensive VM builds on the aggressive one by performing extra type and sanity checks. We can then state the type safety theorem by saying that these checks will never fail if the bytecode is well-typed. This differs from the approach of Wright and Felleisen [1994] that we follow for the source language, where we prove progress and preservation instead. The separation into exec-instr and check-instr allows us to write the 
semantics in a functional rather than relational style at the single-step level. This in turn results in a higher degree of automation in the proofs.

To indicate type errors, we introduce another data type.

$$
\text { datatype 'a type-error = TypeError } \mid \text { Normal 'a }
$$

Similar to Section 3.2, we build on a function check-instr that is lifted over several steps. At the deepest level, we take apart the state, check if the current method exists, feed check-instr with parameters (which are the same as for exec-instr), and check that $p c$ and stack size are valid:

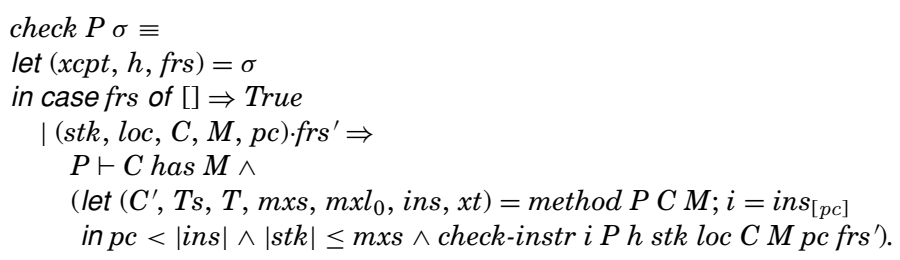

The next level is the one-step execution of the defensive VM, which stops in the case of a type error. If there is no type or other kind of error, it simply calls the aggressive VM:

$$
\text { exec }_{d} P \sigma \equiv \text { if check } P \sigma \text { then Normal (exec } P \sigma \text { ) else TypeError. }
$$

Again, we also define the relational view. This time, it is easier to give two introduction rules for $\stackrel{\text { djvm }}{\longrightarrow}$

$$
\frac{\text { exec }_{d} P \sigma=\text { TypeError }}{P \vdash \text { Normal } \sigma \stackrel{\text { djvm }}{\longrightarrow}{ }_{1} \text { TypeError }} \quad \frac{\text { exec }_{d} P \sigma=\text { Normal }\left\lfloor\sigma^{\prime}\right\rfloor}{P \vdash \text { Normal } \sigma \stackrel{\text { djvm }}{\longrightarrow}{ }_{1} \text { Normal }^{\prime}} \text {. }
$$

We write $\stackrel{\text { djvm }}{\longrightarrow}$ for the reflexive transitive closure of $\stackrel{\text { djvm }}{\longrightarrow} 1$.

It remains to define check-instr, the heart of the defensive Jinja VM. We do so in Figure 19. The IAdd case looks like this:

check-instr IAdd $P$ h stk loc $C_{0} M_{0}$ pc frs $=(1<|s t k| \wedge i s$-Intg $(h d$ stk $) \wedge i s-I n t g(h d(t l s t k)))$

IAdd requires that the stack has at least two entries $(1<|s t k|)$, and that these entries are of type Integer (checked with the is-Intg function). For Load and Store there are no type constraints because they are polymorphic in Jinja. In the Java VM, the definition would be in the style of IAdd, requiring integer for iload, float for fload, and so on. The discriminator functions is-Addr and is-Ref in Figure 19 do the obvious.

Because of its size, we also take a closer look at the instruction Getfield:

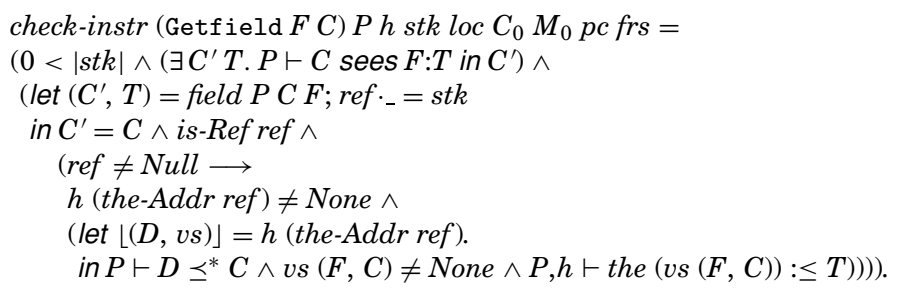




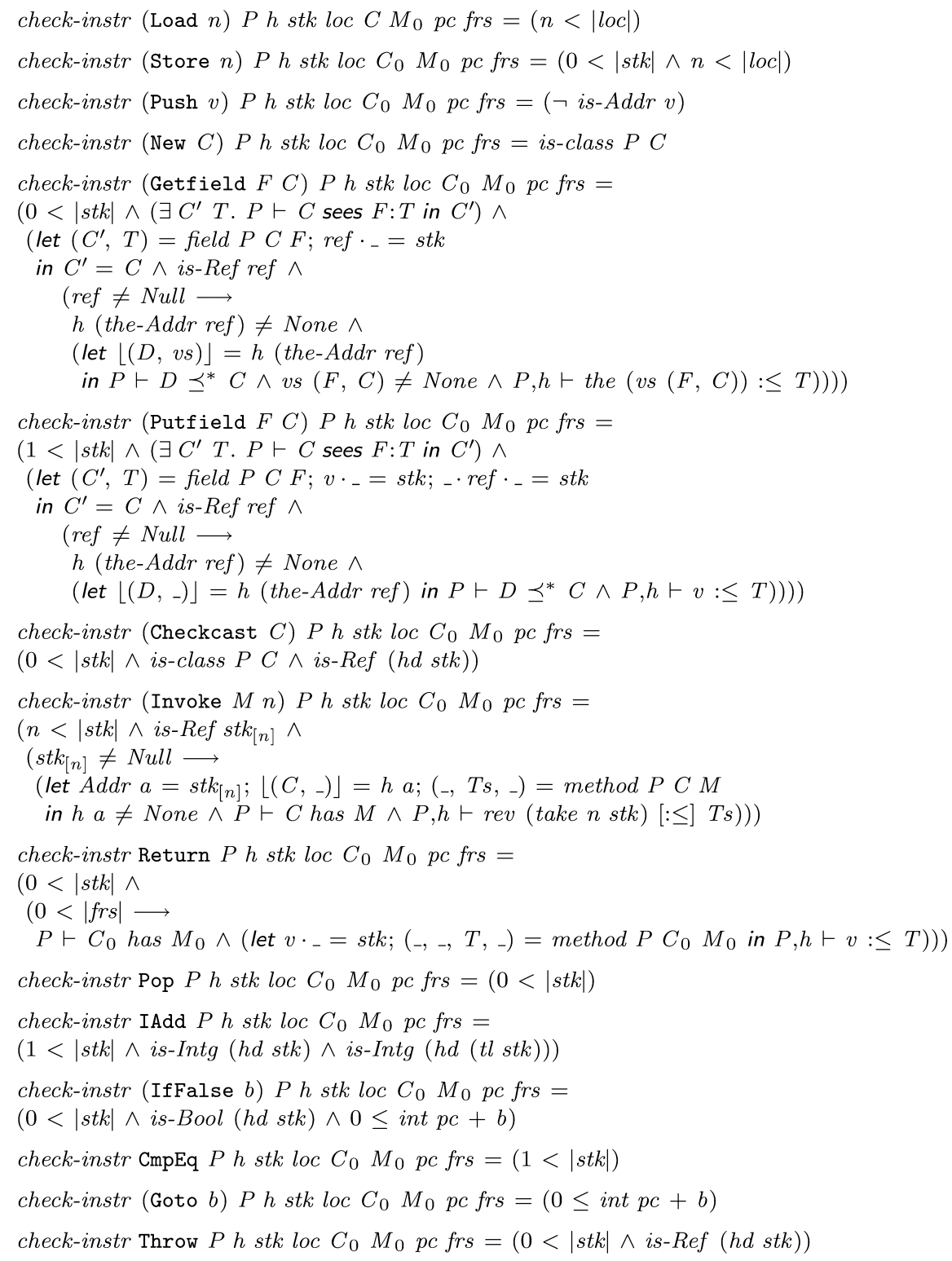

Fig. 19. Type checks in the defensive Jinja VM.

The Getfield $F C$ instruction is supposed to access the object reference on top of the stack, remove it from the stack, and put the value of field $F$ defined in class $C$ onto the stack instead. To ensure that this can work without errors, the first two conjuncts in this definition demand that the stack is large enough to hold the object reference, and that the field $F$ is visible from class $C$. The let-part collects the defining class and the type of field $F$, as well as the object reference. The next line checks that the field is of the right class $\left(C^{\prime}=C\right)$ and 


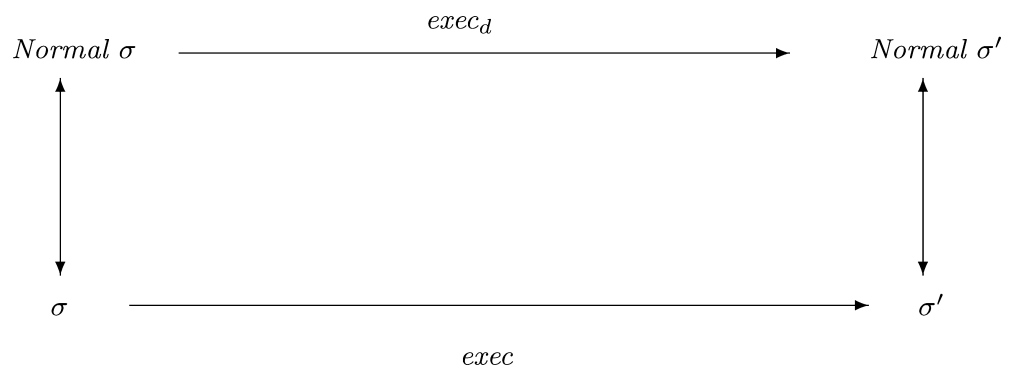

Fig. 20. Aggressive and defensive Jinja VM commute if there are no type errors.

that we are indeed dealing with an object reference. If that reference does not happen to be Null, the heap at position ref must contain an object of a subclass of $C$, the object must contain a field $F$ ( $v s(F, C) \neq N o n e$ ), and the field value must be of the type that was declared for the field. The Putfield instruction works analogously.

It is easy to see that defensive and aggressive VMs have the same operational one-step semantics if there are no type errors.

Theorem 3.1. One-step execution in aggressive and defensive machines commutes if there are no type errors.

$$
\text { exec }_{d} P \sigma \neq \text { TypeError } \Longrightarrow \text { exec }_{d} P \sigma=\text { Normal (exec } P \sigma \text { ) }
$$

Figure 20 depicts this result as a commuting diagram. The proof is trivial (and fully automatic in Isabelle) because the defensive VM is constructed directly from the aggressive one.

For executing programs, we will later also need a canonical start-state. In the Java VM, a program is started by invoking its static main method. We start the Jinja VM by invoking any existing method $M$ (without parameters) in a class $C$. We define the canonical start-state start $P C M$ as the state with exception flag None, a heap start-heap $P$, and a frame stack with one element. The heap start-heap $P$ contains the preallocated system exceptions and is otherwise empty. The single frame has an empty operand stack, the this-pointer set to Null, the rest of the register set filled up with a dummy value arbitrary, the class entry set to $C$, the name to $M$, and the program counter to 0 .

start-state $P C M \equiv$

let $\left(D, T s, T, m x s, m x l_{0}, b\right)=\operatorname{method} P C M$

in (None, start-heap $P,\left[\left([]\right.\right.$, Null-replicate $m x l_{0}$ arbitrary, $\left.\left.\left.C, M, 0\right)\right]\right)$

This concludes the formalisation of the Jinja VM. It will serve as the basis for the proof of type safety to follow.

\subsection{Related Work}

Most closely related to the virtual machine formalisation presented here is the Ph.D. thesis by Klein [2003].The authors Barthe et al. [2001] use the Coq system for a similar formalisation of the Java VM, and Liu and Moore [2003] show one of the most comprehensive executable models of the KVM (a restricted JVM for 


\begin{tabular}{|c|c|}
\hline instruction & registers \\
\hline Load 0 & []$,[$ Class B, Integer $])\rfloor$ \\
\hline Store 1 & $\left\lfloor\left(\left[\begin{array}{ll}\text { Class } & A\end{array}\right],[\right.\right.$ Class B, Err $\left.\left.] \quad\right)\right\rfloor$ \\
\hline Load 0 & $\lfloor(([],[$ Class B, Class A $])\rfloor$ \\
\hline Getfield $F A$ & {$[([$ Class B $],[$ Class B, Class $A])\rfloor$} \\
\hline Goto -3 & $\lfloor([$ Class $A],[$ Class B, Class $A])\rfloor$ \\
\hline
\end{tabular}

Fig. 21. Example of a method well-typing.

embedded devices) in the theorem prover ACL2. Cohen [1997] was the first to propose the concept of a defensive Java VM and to formalise it in a theorem prover. Bertelsen [1997] was one of the first to give a formal semantics for the JVM. Most publications about Java's bytecode verifier contain some form of reference virtual machine, be that in the form of a traditional small step semantics or in an explicitly executable format in a theorem prover. Instead of recounting all of these, here we refer the reader to the overview articles [AlvesFoss 1999; Hartel and Moreau 2001; Nipkow 2003a] and to the related work section on bytecode verification in Section 4.11.

\section{BYTECODE VERIFIER}

The JVM relies on the following assumptions for executing bytecode:

Correct Types. All bytecode instructions are provided with arguments of the type they expect on operand stack, registers, and heap.

No Overflow and Underflow. No instruction tries to retrieve a value from the empty stack, nor puts more elements on the stack than statically specified in the method, nor accesses more registers than statically specified in the method.

Code Containment. The program counter is always within the code array of the method. Specifically, it must not fall off the end of the method's code.

Initialised Registers. All registers apart from the this-pointer and the method parameters must be written to before they are first read. This corresponds to the definite assignment requirement for local variables on the source level.

It is the purpose of the bytecode verifier (BV) to ensure statically that these assumptions are met at any time during execution.

Bytecode verification is an abstract interpretation of bytecode methods: instead of values, we only consider their types. A state type characterises a set of runtime states by giving type information for the operand stack and registers. For example, the first state type in Figure 21 ([],[Class B, Integer]) characterises all states whose stacks are empty, whose register 0 contains a reference to an object of class $B$ (or to a subclass of $B$ ), and whose register 1 contains an integer. A method is called well-typed if we can assign a well-typing to each instruction. A state type ( $S T, L T)$ is a well-typing for an instruction if it is consistent with the successors of the instruction and if the instruction can be executed safely in any state whose stack is typed according to $S T$ and whose registers are typed according to $L T$. In other words, the arguments of the instruction are provided in the correct number, order, and type. We explain consistent in the following. 
The example in Figure 21 shows the instructions on the left and the types of stack elements and registers on the right. The method type is the full righthand side of the table, a state type is one line of it. The type information attached to an instruction characterises the state before execution of that instruction. The $\lfloor\ldots\rfloor$ around each entry means that it was possible to predict some type for each instruction. If one of the instructions had been unreachable, the type entry would have been None. We assume that class $B$ is a subclass of $A$ and that $A$ has a field $F$ of type $A$.

Execution starts with an empty stack and the two registers holding a reference to an object of class $B$ and an integer. The first instruction loads register 0 , a reference to a $B$ object, on the stack. The type information associated with the following instruction may puzzle at first sight: It says that a reference to an $A$ object is on the stack, and that usage of register 1 may produce an error. This means the type information has become less precise but is still correct; a $B$ object is also an $A$ object and an integer is now classified as unusable (Err). The reason for these more general types is that the predecessor of the Store instruction may have either been Load 0 or Goto -3 . Since there exist different execution paths to reach Store, the type information of the two paths has to be merged. The type of the second register is either Integer or Class A, which are incompatible; the only common supertype is Err.

Bytecode verification is the process of inferring the types on the right from the instruction sequence on the left and some initial condition, and of ensuring that each instruction receives arguments of the correct type. Type inference is the computation of a method type from an instruction sequence, and type checking means checking that a given method type fits an instruction sequence.

Figure 21 is an example of a well-typed method: We are able to find a welltyping. If we change the third instruction from Load 0 to Store 0 , the method will not be well-typed. The Store instruction would try to take an element from the empty stack and could therefore not be executed.

In the following, we will formalise the Jinja bytecode verifier by introducing an abstract framework for well-typedness, then we will instantiate this framework in Sections 4.6 and 4.7 to get a description of well-typedness for Jinja VM programs. In addition, we will get an executable program that computes these well-typings in Sections 4.8 and 4.9. Finally, we show in Section 4.10 that execution of well-typed programs is safe in the sense motivated previously.

The typing framework uses semilattices (Section 4.1) and an abstract transfer function to describe well-typings (Section 4.2). After detailing which constraints on the transfer function are necessary to obtain an executable algorithm in Section 4.3 and refining the transfer function to make instantiation easier in Section 4.4, we show an implementation of Kildall's algorithm within the framework (Section 4.5). As the framework itself has already appeared elsewhere [Klein 2003; Klein and Nipkow 2003; Nipkow 2001], we will keep its description brief and only reproduce the main definitions and properties. 


\subsection{Semilattices}

This section introduces the formalisation of the basic lattice-theoretic concepts required for data flow analysis and its application to the JVM.

4.1.1 Partial Orders. Partial orders are formalised as binary predicates. Based on the type synonym ' $a$ ord $={ }^{\prime} a \Rightarrow{ }^{\prime} a \Rightarrow b o o l$ and the notations $x$ 巨r $y \equiv$ $r x y$ and $x \sqsubset_{r} y \equiv x \sqsubseteq_{r} y \wedge x \neq y, r::{ }^{\prime} a$ ord is by definition a partial order iff the predicate order :: ' $a$ ord $\Rightarrow$ bool holds for $r$ :

order $r \equiv$

$(\forall x . x \sqsubseteq r x) \wedge(\forall x y . x \sqsubseteq r y \wedge y \sqsubseteq r x \longrightarrow x=y) \wedge(\forall x y z \cdot x \sqsubseteq r y \wedge y \sqsubseteq r z \longrightarrow x \sqsubseteq r z)$

A partial order $r$ satisfies the ascending chain condition on $A$ if there is no infinite ascending chain $x_{0} \sqsubset_{r} x_{1} \sqsubset_{r} \ldots$ in $A$, and $T$ is called a top element if $x \sqsubseteq r \top$ for all $x$. Instead of "no infinite ascending chain," we require in Isabelle the equivalent "the converse of $r$ is well-founded."

$$
\text { acc } r \equiv w f\left\{(y, x) \mid x \sqsubset_{r} y\right\} \quad \text { top } r \top \equiv \forall x . x \sqsubseteq_{r} \top .
$$

4.1.2 Semilattices. Based on the supremum notation $x \sqcup_{f} y \equiv f x y$ and the two type synonyms 'a binop $={ }^{\prime} a \Rightarrow{ }^{\prime} a \Rightarrow{ }^{\prime} a$ and ' $a$ sl $={ }^{\prime} a$ set $\times{ }^{\prime} a$ ord $\times{ }^{\prime} a$ binop, the tuple $(A, r, f)::$ ' $a$ sl is by definition a semilattice iff the predicate semilat :: 'a $s l \Rightarrow$ bool holds:

semilat $(A, r, f) \equiv$

order $r \wedge$ closed $A f \wedge\left(\forall x \in A . \forall y \in A . x \sqsubseteq r x \sqcup_{f} y\right) \wedge\left(\forall x \in A . \forall y \in A . y \sqsubseteq r x \sqcup_{f} y\right) \wedge$

$\left(\forall x \in A . \forall y \in A . \forall z \in A . x \sqsubseteq r z \wedge y \sqsubseteq r z \longrightarrow x \sqcup_{f} y \sqsubseteq r z\right)$,

where closed $A f \equiv \forall x \in A . \forall y \in A . x \sqcup_{f} y \in A$.

Data flow analysis is usually phrased in terms of infimum semilattices. Here, a supremum semilattice fits better with the intended application where the ordering is the subtype relation and the join of two types is the least common supertype (if it exists).

The next sections look at a few data types and the corresponding semilattices which are required for the construction of the Jinja VM bytecode verifier. The definition of those semilattices follows a pattern: They lift an existing semilattice to a new semilattice with more structure. They extend the carrier set and define two functionals, le and sup, that lift the ordering and supremum operation to the new semilattice. In order to avoid name clashes, Isabelle provides separate name spaces for each theory. Qualified names are of the form Theoryname.localname, and they apply to constant definitions and functions as well as type constructions. So, Err.sup later on refers to the sup functional defined for the error type in Section 4.1.3.

Let $(A, r, f)$ in the following be a semilattice.

4.1.3 The Error Type and Err-Semilattices. Theory Err introduces an error element to model the situation where the supremum of two elements does not exist. It introduces both a data type and an equivalent construction on sets:

datatype 'a err $=E r r \mid O K^{\prime} a \quad \operatorname{err} A \equiv\{\operatorname{Err}\} \cup\{O K x \mid x . x \in A\}$

ACM Transactions on Programming Languages and Systems, Vol. 28, No. 4, July 2006. 
The additional $x$. in $\{O K x \mid x . x \in A\}$ tells Isabelle that $x$ is a bound variable. It is used if the set comprehension is of the form $\{f x \mid x . P x\}$ rather than just $\{x \mid P x\}$. An ordering $r$ on ' $a$ can be lifted to 'a err by making $E r r$ the top element:

$$
\begin{array}{ll}
\text { le } r(\mathrm{OK} x)(\mathrm{OK} y) & =x \sqsubseteq r y \\
\text { le } r_{\_} E r r & =\text { True } \\
\text { le } r \text { Err }\left(\mathrm{OK}_{-}\right) & =\text {False }
\end{array}
$$

LEMMa 4.1. If acc $r$ then acc (le $r$ ).

The following lifting functional is frequently useful:

$$
\begin{array}{ll}
\text { lift }_{2} f(O K x)(O K y) & =f x y \\
\text { lift }_{2} f E r r & =E r r \\
\text { lift }_{2} f_{-} E r r & =E r r
\end{array}
$$

This leads to the notion of an err-semilattice. It is a variation of a semilattice with a top element. Because the behaviour of the ordering and the supremum on the top element is fixed, it suffices to say how ordering and supremum behave on nontop elements. Thus, we can represent a semilattice with top element $E r r$ compactly by a triple of type esl:

$$
\text { 'a ebinop }=' a \Rightarrow \text { 'a } \Rightarrow \text { 'a err } \quad \text { 'a esl }=\text { 'a set } \times \text { 'a ord } \times \text { 'a ebinop. }
$$

Conversion between the types $s l$ and $e s l$ is easy:

$$
\begin{array}{ll}
\text { esl }:: ' a \text { sl } \Rightarrow \text { 'a esl } & \text { sl :: 'a esl } \Rightarrow \text { 'a err sl } \\
\text { esl }(A, r, f) \equiv(A, r, \lambda x y . O K(f x y)) & \text { sl }(A, r, f) \equiv\left(\text { err } A, \text { le } r, l_{\text {lift }} f\right) .
\end{array}
$$

A tuple $L$ :: 'a esl is by definition an err-semilattice iff $s l L$ is a semilattice. Conversely, we have Lemma 4.2.

Lemma 4.2. If semilat $L$ then err-semilat (esl $L$ ).

The supremum operation of $\operatorname{sl}($ esl $L)$ is useful on its own:

$$
\sup f \equiv \operatorname{lift}_{2}\left(\lambda x y . O K\left(x \sqcup_{f} y\right)\right) .
$$

4.1.4 The Option Type. Theory Opt uses the type option and introduces the set opt as dual to set err,

$$
\text { opt } A \equiv\{\text { None }\} \cup\{\lfloor y\rfloor \mid y \cdot y \in A\} .
$$

an ordering that makes None the bottom element, and a corresponding supremum operation:

$$
\begin{aligned}
& \text { le } r\lfloor x\rfloor\lfloor y\rfloor=x \sqsubseteq r y \\
& \text { le } r \text { None }=\text { True } \\
& \text { le } r\left\lfloor \_ \text {None }=\right.\text { False } \\
& \text { sup } f\lfloor x\rfloor\lfloor y\rfloor=(\text { case } f x \text { y of Err } \Rightarrow \text { Err } \mid O K z \Rightarrow O K\lfloor z\rfloor) \\
& \text { sup } z \text { None }=\text { OKz } \\
& \text { sup f None } z=O K z .
\end{aligned}
$$

Lemma 4.3. Let esl $(A, r, f)=($ opt $A$, le $r$, sup $f$ ). If err-semilat L, then err-semilat (esl L). 
It is possible to define an $s l$ that lifts a semilattice to an option semilattice, but we only use the esl version below.

Lemma 4.4. If acc $r$, then acc (le $r$ ).

4.1.5 Products. Theory Product provides what is known as the coalesced product, where the top elements of both components are identified. In terms of err-semilattices, this is:

$$
\begin{aligned}
& \text { esl :: 'a esl } \Rightarrow \text { 'b esl } \Rightarrow\left({ }^{\prime} a \times{ }^{\prime} b\right) \text { esl } \\
& \text { esl }\left(A, r_{A}, f_{A}\right)\left(B, r_{B}, f_{B}\right) \equiv\left(A \times B \text {, le } r_{A} r_{B} \text {, sup } f_{A} f_{B}\right) \\
& \text { le }:: \text { ' } a \text { ord } \Rightarrow ' b \text { ord } \Rightarrow\left({ }^{\prime} a \times ' b\right) \text { ord } \\
& \text { le } r_{A} r_{B}\left(a_{1}, b_{1}\right)\left(a_{2}, b_{2}\right) \equiv a_{1} \sqsubseteq r_{A} a_{2} \wedge b_{1} \sqsubseteq r_{B} b_{2} \\
& \text { sup :: 'a ebinop } \Rightarrow b^{\prime} b \text { ebinop } \Rightarrow(' a \times ' b) \text { ebinop } \\
& \text { sup } f g\left(a_{1}, b_{1}\right)\left(a_{2}, b_{2}\right) \equiv \operatorname{Err} . \sup (\lambda x y .(x, y))\left(a_{1} \sqcup_{f} a_{2}\right)\left(b_{1} \sqcup_{g} b_{2}\right) \text {. }
\end{aligned}
$$

Note that $x$ is used both on the type and the set level.

LEMMA 4.5. If err-semilat $L_{1}$ and err-semilat $L_{2}$, then err-semilat (esl $\left.L_{1} L_{2}\right)$.

LEMMA 4.6. If acc $r_{A}$ and acc $r_{B}$, then acc $\left(\right.$ le $\left.r_{A} r_{B}\right)$.

4.1.6 Lists of Fixed Length. Theory Listn provides the concept of lists of a given length over a given set. In HOL, this is formalised as a set rather than a type:

$$
\text { list } n A \equiv\{x s|| x s \mid=n \wedge \text { set } x s \subseteq A\} .
$$

This set can be turned into a semilattice in a componentwise manner, essentially viewing it as an $n$-fold Cartesian product:

$$
\begin{aligned}
& s l:: n a t \Rightarrow \text { 'a } s l \Rightarrow \text { 'a list sl le :: 'a ord } \Rightarrow \text { 'a list ord } \\
& \operatorname{sln}(A, r, f) \equiv\left(\text { list } n A \text {, le } r, \operatorname{map}_{2} f\right) \quad \text { le } r \equiv \operatorname{list} \text {-all }_{2}\left(\lambda x y . x \sqsubseteq_{r} y\right) \text {, }
\end{aligned}
$$

where $\operatorname{map}_{2}: \because\left(^{\prime} a \Rightarrow{ }^{\prime} b \Rightarrow{ }^{\prime} c\right) \Rightarrow{ }^{\prime} a$ list $\Rightarrow{ }^{\prime} b$ list $\Rightarrow{ }^{\prime} c$ list and list-all $2::\left({ }^{\prime} a \Rightarrow{ }^{\prime} b\right.$ $\Rightarrow b o o l) \Rightarrow{ }^{\prime} a$ list $\Rightarrow$ 'b list $\Rightarrow$ bool are the obvious functions. To follow, we use the notation $x s$ [ $\left.\subseteq_{r}\right] y s$ for $x s \sqsubseteq_{l e} r y s$.

LeMma 4.7. If semilat $L$, then semilat $(s \ln L)$.

LEMMA 4.8. If order $r$ and acc $r$, then acc (le $r$ ).

In case we want to combine lists of different lengths, or if the supremum on the elements of the list may return Err (not to be confused with Err.sup, the sup functional defined in Theory Err, Section 4.1.3), the following function is useful:

$$
\begin{aligned}
& \text { sup }::\left({ }^{\prime} a \Rightarrow{ }^{\prime} b \Rightarrow{ }^{\prime} \text { c err }\right) \Rightarrow \text { 'a list } \Rightarrow{ }^{\prime} b \text { list } \Rightarrow \text { c list err } \\
& \text { sup } f x s \text { ys } \equiv \text { if }|x s|=|y s| \text { then coalesce ( } \text { map }_{2} f \text { xs ys) else Err } \\
& \text { coalesce }[]=O K[] \\
& \text { coalesce }(e \cdot e s)=\text { Err.sup }(\lambda x x s . x \cdot x s) \text { e (coalesce es }) \text {. }
\end{aligned}
$$




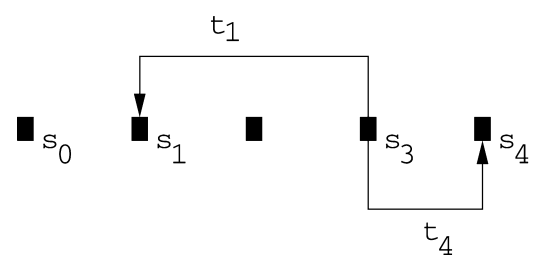

Fig. 22. Data flow graph for step $3 s_{3}=\left[\left(1, t_{1}\right),\left(4, t_{4}\right)\right]$.

This corresponds to the coalesced product. In the following, we also need the structure of all lists up to a specific length:

$$
\begin{aligned}
& \text { upto-esl }:: \text { nat } \Rightarrow \text { 'a esl } \Rightarrow \text { 'a list esl } \\
& \text { upto-esl } n(A, r, f) \equiv\left(\bigcup_{i \leq n} \text { list } i A \text {, le } r \text {, sup } f\right) .
\end{aligned}
$$

Lemma 4.9. If err-semilat $L$, then err-semilat (upto-esl $m L$ ).

\subsection{Well-Typings}

This section describes well-typings abstractly. On this abstract level, there is no need yet to talk about the instruction sequences themselves. They will be hidden inside a function that characterises their behaviour. This function and a semilattice form the parameters of the model.

Data flow analysis and type systems are based on an abstract view of the semantics of a program in terms of types instead of values. At this level, programs are sequences of instructions, and the semantics can be characterised by a function step $:: p c \Rightarrow ' s \Rightarrow(p c \times ' s)$ list, where $p c$ is an abbreviation for nat. It is the abstract execution function: step $p s$ provides the results of executing the instruction at $p$, starting in state $s$, together with the positions where execution continues. Contrary to the usual concept of transfer function or flow function in the literature, step $p$ not only provides the result, but also the structure of the data flow graph at position $p$. This is best explained through an example. Figure 22 depicts the information we get when step $3 s_{3}$ returns the list $\left[\left(1, t_{1}\right),\left(4, t_{4}\right)\right]$ : Executing the instruction at position 3 with state type $s_{3}$ may lead to position 1 in the graph with result $t_{1}$, or to position 4 with result $t_{4}$.

Note that the length of the list and the target instructions do not only depend on the source position $p$ in the graph, but also on the value of $s$. It is possible for the structure of the data flow graph to dynamically change in the iteration process of the analysis. It may not change freely, however. Section 4.3 will introduce certain constraints on the step function that the analysis needs in order to succeed.

Data flow analysis is concerned with solving data flow equations, which are systems of equations involving the flow functions over a semilattice. In this case, step is the flow function and 's the semilattice. Instead of an explicit formalisation of the data flow equation, it suffices to consider certain prefixed points. To that end we define what it means that a method type $\tau s::$ 's list is stable at $p$ :

stable $\tau s p \equiv \forall(q, \tau) \in \operatorname{set}\left(\right.$ step $\left.p \tau s_{[p]}\right) . \tau \sqsubseteq r \tau s_{[q]}$. 
Stability induces the notion of a method type $\tau s$ as being a well-typing w.r.t. step:

$$
w t \text {-step } \tau s \equiv \forall p<|\tau s| . \tau s_{[p]} \neq \top \wedge \text { stable } \tau s p .
$$

$\top$ is assumed to be a special element in the state space (the top element of the ordering). It indicates a type error.

An instruction sequence is well-typed if there is a well-typing $\tau s$ such that $w t$-step $\tau s$.

\subsection{Constraints on the Transfer Function}

This section defines constraints on the transfer function that the data flow algorithm in Section 4.5 requires to succeed.

The transfer function step is called monotone up to $n$ iff the following holds:

$$
\text { mono } n \equiv \forall \tau \in A . \forall p<n . \forall \tau^{\prime} . \tau \sqsubseteq r \tau^{\prime} \longrightarrow \operatorname{set}(\operatorname{step} p \tau)\{\sqsubseteq r\} \operatorname{set}\left(\text { step } p \tau^{\prime}\right) \text {, }
$$

where

$$
A\{\sqsubseteq r\} B \equiv \forall(p, \tau) \in A . \exists \tau^{\prime} .\left(p, \tau^{\prime}\right) \in B \wedge \tau \sqsubseteq r \tau^{\prime} .
$$

This means if we increase the state type $\tau$ at a position $p$, the data flow graph may have more edges (but not less), and the result at each edge may increase (but not decrease).

If for all $p<n$ and all $\tau$ the position components of step $p \tau$ are less than $n$, then step is bounded by $n$. This expresses that from below instruction $n$, instruction $n$ and beyond are unreachable; control never leaves the list of instructions below $n$.

$$
\text { bounded } n \equiv \forall p<n . \forall \tau . \forall\left(q, \tau^{\prime}\right) \in \operatorname{set}(\text { step } p \tau) . q<n
$$

If for all $p<n$ and $\tau \in A$ the values that step $p \tau$ returns are again in $A$, then step preserves $A$ up to $n$ :

$$
\text { preserves } n \equiv \forall \tau \in A . \forall p<n . \forall\left(q, \tau^{\prime}\right) \in \operatorname{set}(\operatorname{step} p \tau) . \tau^{\prime} \in A
$$

\subsection{Refining the Transfer Function}

The single transfer function step of Section 4.2 is compact and convenient for describing the abstract typing framework. For a large instantiation, however, it carries too much information in one place to be modular and intuitive. We will therefore first refine step into a part app for applicability and a part eff for the effect of instructions, and then instantiate these parts in Section 4.7. Furthermore, the state space 's will be of the form ' $t$ err for a suitable type ' $t$, in which case the error element $\top$ is $\operatorname{Err}$ itself. Given $a p p:: p c \Rightarrow ' t \Rightarrow b o o l$, eff $:: p c \Rightarrow t \Rightarrow(p c \times ' t)$ list, and $n::$ nat (the size of the method type), step is defined as follows:

$$
\begin{array}{ll}
\text { step } p \text { Err } & =\text { error } \\
\text { step } p(O K \tau) & =(\text { if app } p \tau \text { then map-snd OK }(\text { eff } p \tau) \text { else error }) \\
\text { error } & \equiv \operatorname{map}(\lambda x .(x, \text { Err }))[0 \ldots<n] \\
\text { map-snd } f & \equiv \operatorname{map}(\lambda(x, y) .(x, f y)) .
\end{array}
$$


The function error is used to propagate the error element $E r r$ to every position in the method type.

If we take the semilattice $(A, r, f)$ to be an err-semilattice and the order $r$ to be of the form $l e r^{\prime}$, we can similarly refine the notion of a well-typing w.r.t. step to a well-typing w.r.t. app and eff:

$$
w t \text {-app-eff } \tau s \equiv \forall p<|\tau s| \text {.app p } \tau s_{[p]} \wedge\left(\forall(q, \tau) \in \operatorname{set}\left(\operatorname{eff} p \tau s_{[p]}\right) . \tau \sqsubseteq_{r^{\prime}} \tau s_{[q]}\right) .
$$

This is very natural: Every instruction is applicable in its start state, and the effect is compatible with the state expected by all successor instructions.

Function step, composed of app and eff as defined previously, has type $p c \Rightarrow$ 't err $\Rightarrow\left(p c \times{ }^{\prime} t\right.$ err $)$ list. This is an instance of the type that the stability predicates in Section 4.2 expect. If we furthermore set $n$ to $|\tau s|$, we get the following lemma.

LEMMA 4.10. If the composed function step is bounded by $n=|\tau s|$, then wt-app-eff and wt-step coincide:

$$
\text { bounded }|\tau s| \Longrightarrow w t \text {-step (map OK } \tau s)=w t \text {-app-eff } \tau s \text {. }
$$

\subsection{Kildall's Algorithm}

A well-typing is a witness of well-typedness in the sense of stability. Now we turn to the problem of computing such a witness. This is precisely the task of a bytecode verifier: It computes a method type such that the absence of $T$ in the result means the method is well-typed. Formally, a function $b c v::$ 's list $\Rightarrow$ 's list is a bytecode verifier w.r.t. $n::$ nat and $A$ :: 's set iff

$$
\begin{aligned}
& \forall \tau s_{0} \in \text { list } n A .\left(\forall p<n .\left(b c v \tau s_{0}\right)_{[p]} \neq \top\right) \\
& =\left(\exists \tau s \in \text { list } n A . \tau s_{0}\left[\complement_{r}\right] \tau s \wedge w t \text {-step } \tau s\right) .
\end{aligned}
$$

The notation $\left[\sqsubseteq_{r}\right]$ lifts $\sqsubseteq_{r}$ to lists (see also Section 4.1.6), and $\top$ is the top element of the semilattice. In practise, $b c v \tau s_{0}$ itself will be the well-typing, and it will also be the least well-typing. However, it is simpler not to require this.

This section first defines and then verifies a functional version of Kildall's algorithm [Kildall 1973; Muchnick 1997], a standard data flow analysis tool. In fact, the description of bytecode verification in the official JVM specification [Lindholm and Yellin 1999, 129-130] is essentially Kildall's algorithm, an iterative computation of the solution to the data flow problem. The main loop operates on a method type $\tau s$ and a worklist $w::$ pc set. The worklist contains the indices of those elements of $\tau s$ that have changed and whose changes still need to be propagated to their successors. Each iteration picks an element $p$ from $w$, executes instruction number $p$, and propagates the new states to the successor instructions of $p$. Iteration terminates once $w$ becomes empty: In each iteration $p$ is removed, but new elements can be added to $w$. The algorithm is expressed in terms of a predefined while-combinator of type $(' a \Rightarrow b o o l) \Rightarrow\left({ }^{\prime} a \Rightarrow^{\prime} a\right) \Rightarrow{ }^{\prime} a \Rightarrow^{\prime} a$, which satisfies the recursion equation

while $b c s=($ if $b s$ then while $b c(c s)$ else $s)$. 
The term while ( $\lambda s . b s)(\lambda s . c s)$ is the functional counterpart of the imperative program while $b(s)$ do $s:=c(s)$. The main loop can now be expressed as

iter $\tau s w \equiv$

while $(\lambda(\tau s, w) . w \neq\{\})$

$\left(\lambda(\tau s, w)\right.$. let $p=S O M E p \cdot p \in w$ in propa $\left(\right.$ step $\left.\left.p \tau s_{[p]}\right) \tau s(w-\{p\})\right)$

$(\tau s, w)$.

Since the choice $S O M E p \cdot p \in w$ in iter is guarded by $w \neq\{\}$, we know that there is a $p \in w$. An implementation is free to choose whichever element it wants.

Propagating the results $q s$ of executing instruction number $p$ to all successors is expressed by the primitive recursive function propa:

propa []$\tau s w=(\tau s, w)$

propa $\left(q^{\prime} \cdot q s\right) \tau s w=$

(let $(q, \tau)=q^{\prime} ; u=\tau \sqcup_{f} \tau s_{[q]} ; w^{\prime}=$ if $u=\tau s_{[q]}$ then $w$ else $\{q\} \cup w$

in propa $\left.q s(\tau s[q:=u]) w^{\prime}\right)$.

In the terminology of the official JVM specification [Lindholm and Yellin 1999, 130], $\tau$ is merged with the state of all successor instructions $q$, that is, the supremum is computed. If this results in a change of $\tau s_{[q]}$, then $q$ is inserted into $w$.

Kildall's algorithm is simply a call to iter where the worklist is initialised with the set of unstable indices; upon termination, we project on the first component:

$$
\text { kildall } \tau s \equiv \text { sst (iter } \tau s\{p|p<| \tau s \mid \wedge \neg \text { stable } \tau s p\}) .
$$

The key theorem is that Kildall's algorithm is a bytecode verifier as defined previously.

THEOREM 4.11. If $(A, r, f)$ is a semilattice, $r$ meets the ascending chain condition on $A$, and step is monotone, preserving, and bounded w.r.t. A and n, then kildall is a bytecode verifier w.r.t. A and $n$.

Proof. The correctness proof proceeds along the following lines: (1) Given the assumptions of Theorem 4.11, kildall is a total function. The work list either becomes smaller, or if new positions are introduced, the elements they point to are larger than the element at the position that was taken out. Since there are no infinitely ascending chains in $r$, the algorithm must terminate; and (2) The following are invariants of the analysis (mainly because of monotonicity of step): all positions not in the worklist are stable, and the computed method type is always between the start value $\tau s_{0}$ and any well-typing $\tau s$ which is stable everywhere and satisfies $\tau s_{0} \sqsubseteq_{r} \tau s$. Upon termination the worklist is empty, and kildall $\tau s_{0}$ is stable everywhere. With (1) and (2), Theorem 4.11 follows easily. The implication from left to right holds with the result of the algorithm as witness. The result is stable, it does not contain $T$ (by assumption), and it is above $\tau s_{0}$ because of (2). The implication from right to left holds because $w t$-step implies that $\tau s$ is stable and does not contain $T$. Because of (2), kildall $\tau s_{0}$ is smaller than $\tau s$, hence, it does not contain $T$ either. 
This specification of Kildall's algorithm is executable; the worklist (in the specification a set) can be implemented by a list, the $S O M E$ operator by $h d$.

\subsection{Semilattice for the Jinja VM}

This section takes the first step to instantiate the framework of Sections 4.1 through 4.5. It defines the semilattice structure on which Jinja's bytecode verifier builds. It begins by turning the Jinja types $t y$ into a semilattice in Theory SemiType, to follow. We can then use the abstract combinators of Section 4.1 to construct the stack and register structure.

The carrier set types is easy: the set of all types declared in the program.

$$
\text { types } P=\{T \mid \text { is-type } P T\}
$$

The order is the standard subtype ordering $\leq$ of Jinja and the supremum operation follows it.

$$
\begin{aligned}
& \text { sup }:: \text { jum-prog } \Rightarrow t y \Rightarrow t y \Rightarrow \text { ty err } \\
& \text { sup } P N T(\text { Class } C)=O K(\text { Class } C) \\
& \text { sup } P(\text { Class } C) N T=O K(\text { Class } C) \\
& \text { sup } P(\text { Class } C)(\text { Class } D)=O K(\text { Class }(\text { lub P C D })) \\
& \text { sup } P t_{1} t_{2}=\left(\text { if } t_{1}=t_{2} \text { then } O K t_{1} \text { else Err }\right)
\end{aligned}
$$

The $l u b$ function (not shown here) computes the least upper bound of two classes by walking up the class hierarchy until one is a subclass of the other. Since every class is a subclass of Object in a well-formed program (see also $w f$-prog in Section 2.4), this least upper bound is guaranteed to exist.

With SemiType esl $P \equiv($ types $P, \lambda x y . P \vdash x \leq y$, sup $P$ ) we have proved the following theorem.

THEOREM 4.12. If $P$ is well-formed, then SemiType.esl is an err-semilattice and the subtype ordering $\leq$ satisfies the ascending chain condition:

$$
\begin{aligned}
& \text { wf-prog }-P \Longrightarrow \text { err-semilat (SemiType.esl } P) \\
& \text { wf-prog }-P \Longrightarrow \text { acc }(\lambda x y . P \vdash x \leq y) .
\end{aligned}
$$

Proof. The proof is easy; it is obvious that $\leq$ is transitive and reflexive. If $P$ is well-formed, $\leq$ is also antisymmetric, hence a partial order. It satisfies the ascending chain condition because if $P$ is well-formed, the class hierarchy is a tree with Object at its top. We have already argued that sup is well defined, and it is easy to see that it is closed w.r.t. types P. Hence, SemiType.esl $P$ is an err-semilattice.

We can now construct the stack and register structure. State types in the Jinja BV are the same as the example in Figure 21: Values on the operand stack must always contain a known type $t y$, and values in the local variables may be of an unknown type and therefore be unusable (encoded by $E r r$ ). To handle unreachable code, the BV needs an additional option layer: If None occurs in the well-typing, the corresponding instruction is unreachable. On the HOL-type level:

$$
\begin{array}{lll}
\text { types } & t y_{s}=t y \text { list } & t y_{l}=t y \text { err list } \\
& t y_{i}=t y_{s} \times t y_{l} & t y_{i}{ }^{\prime}=t y_{i} \text { option. }
\end{array}
$$


The types of the stack and the local variables are $t y_{s}$ and $t y_{l}$, respectively. We call ty $y_{i}$ the instruction type, and let the term state type refer to either of $t y_{i}$ or $t y_{i}{ }^{\prime}$. It will be clear from the context which of these is meant.

The data flow analysis also needs to indicate type errors during the algorithm (see also Section 4.4), so we arrive at $t y_{i}{ }^{\prime}$ err for the semilattice construction.

Turning $t y_{i}{ }^{\prime}$ err into a semilattice is easy because all of its constituent types are (err-)semilattices. The expression stacks form a semilattice because the supremum of stacks of different size is $E r r$; the local variables form a semilattice because their number $m x l$ is fixed:

$$
\begin{aligned}
& \text { stk-esl }:: \text { jum-prog } \Rightarrow \text { nat } \Rightarrow \text { ty } y_{s} \text { esl } \\
& \text { stk-esl } P \text { mxs } \equiv \text { upto-esl } m x s(\text { SemiType.esl P }) \\
& \text { loc-sl }:: \text { jum-prog } \Rightarrow \text { nat } \Rightarrow \text { ty } y_{l} \text { sl } \\
& \text { loc-sl } P \text { mxl } \equiv \text { Listn.sl } m x l(\text { Err.sl (SemiType.esl P) }) .
\end{aligned}
$$

Stack and local variables are combined into a coalesced product via Product.esl and then embedded into option and err to create the final semilattice for 's $=t y_{i}{ }^{\prime}$ err:

$$
\begin{aligned}
& \text { sl :: jum-prog } \Rightarrow \text { nat } \Rightarrow \text { nat } \Rightarrow t y_{i}{ }^{\prime} \text { err sl } \\
& \text { sl P } m x s \text { mxl } \equiv \operatorname{Err.sl}(\text { Opt.esl (Product.esl (stk-esl P } m x s)(\text { Err.esl (loc-sl P } m x l)))) .
\end{aligned}
$$

It is useful in the following with special notation $\leq^{\prime}$ for the ordering on $t y_{i}{ }^{\prime}$.

Combining the theorems about the various (err-)semilattice constructions involved in the definition of $s l$ (starting from Theorem 4.12, using Lemmas 4.1 to 4.9), it is easy to prove

Corollary 4.13. If $P$ is well-formed, then sl is a semilattice. Its order (written le $P(m x s m x l)$ satisfies the ascending chain condition:

$$
\begin{aligned}
& \text { wf-prog }-P \Longrightarrow \text { semilat }(\text { sl } P \text { mxs } m x l) \\
& \text { wf-prog } P \Longrightarrow \text { acc }(\text { le } P \text { m } m x \text { s } m \text { ). }
\end{aligned}
$$

\subsection{Applicability and Effect Instantiated}

In this section we instantiate $a p p$ and eff from Section 4.4 for the instruction set of the Jinja VM. As for the source language, we have divided the definitions into one part for normal execution and one part for exceptional.

Since the BV verifies one method at a time, we can see the context of a method and a program as fixed for the definition. The context consists of the following values:

$$
\begin{array}{lll}
P & :: \text { jum-prog } & \text { the program, } \\
C^{\prime}:: \text { cname } & \text { the class the method we are verifying is declared in, } \\
m x s:: \text { nat } & \text { maximum stack size of the method, } \\
m x l:: \text { nat } & \text { size of the register set for local variables, } \\
T s:: \text { ty list } & \text { types of the parameters of the method, } \\
T_{r}:: \text { ty } & \text { return type of the method, } \\
\text { is }:: \text { instr list } & \text { instructions of the method, } \\
x t & :: \text { ex-table } & \text { exception handler table of the method, }
\end{array}
$$




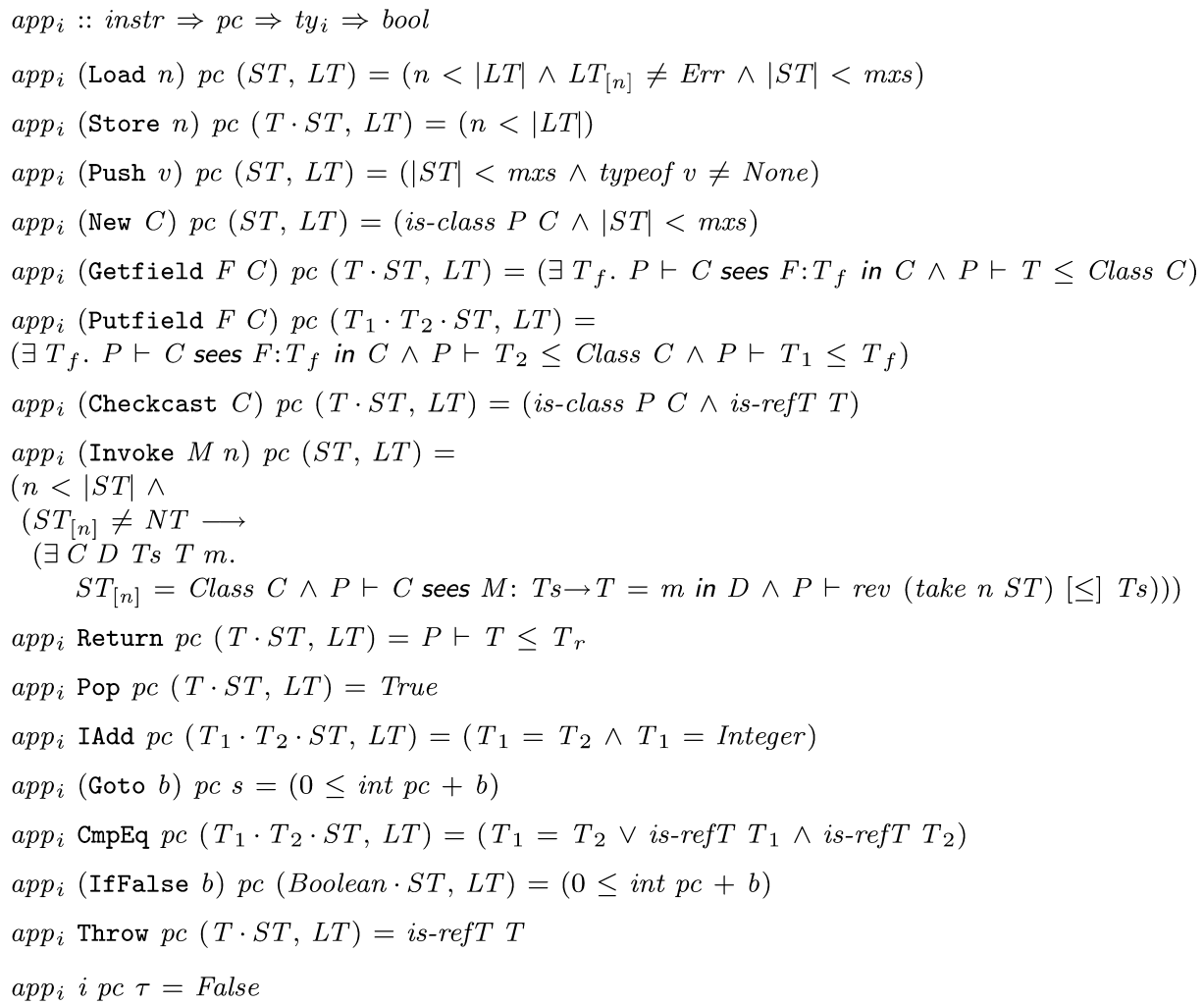

Fig. 23. Applicability of instructions.

The context variables are proper parameters of eff and app in the Isabelle formalisation. We treat them as global here to spare the reader endless parameter lists in each definition. Formally, we use Isabelle's locale mechanism to hide these parameters in the presentation. Ballarin [2003] describes locales in detail.

4.7.1 Normal Execution. We begin with the definition of applicability for normal execution. The intermediate function $a p p_{i}$, defined in Figure 23, works on $t y_{i}$, and $a p p$ will later lift it to $t y_{i}{ }^{\prime}$. The definition is parallel to check-instr in Section 3.3, it just works on types instead of on values. The definition is smaller than that of check-instr because some of the conditions cannot be expressed at the type level alone. These conditions are the ones that access the heap or the frame stack (most notable in the Getfield, Putfield, and Return instructions). It will be the responsibility of the type safety proof to show that the BV still manages to guarantee that all checks in the defensive machine are successful.

Let's take a closer look at the IAdd example again:

$$
a p p_{i} \text { IAdd } p c\left(T_{1} \cdot T_{2} \cdot S T, L T\right)=\left(T_{1}=T_{2} \wedge T_{1}=\text { Integer }\right) .
$$

This is completely parallel to the defensive machine. The pattern on the lefthand side ensures that there are at least two elements on the stack, and the righthand side requires that they are both integers. 


$$
\begin{array}{ll}
\text { succs }: \text { instr } \Rightarrow t y_{i} \Rightarrow p c & \Rightarrow p c \text { list } \\
\text { succs }(\text { IfFalse } b) \tau p c & =[p c+1, \text { nat }(\text { int } p c+b)] \\
\text { succs }(\text { Goto } b) \tau p c & =[\text { nat }(\text { int } p c+b)] \\
\text { succs }(\text { Invoke } M n) \tau p c & =\left(\text { if }(\text { fst } \tau)_{[n]}=N T \text { then }[] \text { else }[p c+1]\right) \\
\text { succs } \text { Return } \tau p c & =[] \\
\text { succs Throw } \tau p & =[] \\
\text { succs } i \tau p c & =[p c+1]
\end{array}
$$

Fig. 24. Successor program counters for the non-exceptional case.

$$
\begin{aligned}
& \text { eff }{ }_{i}:: \text { instr } \Rightarrow t y_{i} \Rightarrow t y_{i} \\
& \operatorname{eff}_{i}(\text { Load } n)(S T, L T) \quad=\left(\text { ok-val } L T_{[n]} \cdot S T, L T\right) \\
& \text { eff }_{i}(\text { Store } n)(T \cdot S T, L T) \quad=(S T, L T[n:=O K T]) \\
& \text { eff }_{i}(\text { Push } v)(S T, L T) \quad=(\text { the }(\text { typeof } v) \cdot S T, L T) \\
& \text { eff }_{i}(\text { New } C)(S T, L T) \quad=(\text { Class } C \cdot S T, L T) \\
& \text { eff }_{i}(\text { Getfield } F C)(T \cdot S T, L T) \quad=(\text { snd }(\text { field } P C F) \cdot S T, L T) \\
& \text { eff } \left._{i} \text { (Putfield } F C\right)\left(T_{1} \cdot T_{2} \cdot S T, L T\right)=(S T, L T) \\
& \text { eff } \left._{i} \text { (Checkcast } C\right)(T \cdot S T, L T)=(\text { Class } C \cdot S T, L T) \\
& \text { eff }{ }_{i} \text { Pop }(T \cdot S T, L T) \quad=(S T, L T) \\
& \text { eff } i \text { IAdd }\left(T_{1} \cdot T_{2} \cdot S T, L T\right) \quad=(\text { Integer } \cdot S T, L T) \\
& \text { eff }_{i} \text { (Goto } n \text { ) } s \\
& \text { eff } i \text { CmpEq }\left(T_{1} \cdot T_{2} \cdot S T, L T\right) \quad=(\text { Boolean } \cdot S T, L T) \\
& \text { eff }_{i}(\text { IfFalse } b)\left(T_{1} \cdot S T, L T\right) \quad=(S T, L T) \\
& \text { eff }_{i}(\text { Invoke } M n)(S T, L T) \quad=\text { let Class } C=S T_{[n]} \text {; } \\
& \left({ }_{-},-, T_{r},{ }_{-}\right)=\text {method } P C M \\
& \text { in }\left(T_{r} \cdot \operatorname{drop}(n+1) S T, L T\right)
\end{aligned}
$$

Fig. 25. Effect of instructions on the state type.

The nonexceptional effect of instructions eff $i$ is equally simple. First, we calculate the successor program counters in Figure 24, and after that, the effect of the instruction on the type level in Figure 25.

The successors are easy; most instructions simply proceed to $p c+1$. The relative jumps in IfFalse and Goto use the nat and int functions to convert the HOL-types nat to int, and vice versa. Return and Throw have no successors in the same method (for the nonexceptional case). The Invoke $n M$ instruction has no normal successor if the the stack at position $n$ contains $N T$-it will always throw a NullPointer exception in this case. It is different from Getfield and Putfield because they have enough information in the instruction itself to determine the effect. Invoke, on the other hand, must rely on computed information at $S T_{[n]}$ to determine the return type of the method. In the Java VM, the Invoke instruction contains the static class of the method (and is thus easier to handle).

The effect $e f f_{i}$ on $t y_{i}$ is shown in Figure 25. The destructor ok-val is defined by $o k$-val $(O K x)=x$.

The IAdd instruction is in this case:

$$
\text { eff } i_{i} \text { IAdd }\left(T_{1} \cdot T_{2} \cdot S T, L T\right)=(\text { Integer } \cdot S T, L T) .
$$

Again, as befits an abstract interpretation, the definition is completely parallel to the operational semantics, this time to exec-instr of the aggressive machine. 


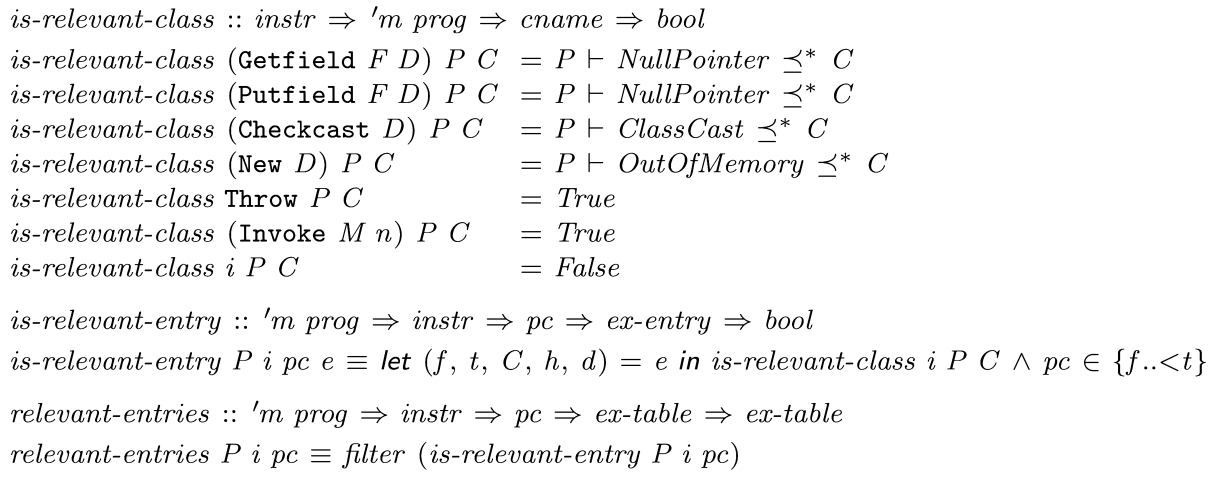

Fig. 26. Finding relevant exception handlers.

The next step is combining eff $i$ and succs:

$$
\begin{aligned}
& \text { norm-eff }:: \text { instr } \Rightarrow p c \Rightarrow t y_{i} \Rightarrow\left(p c \times t y_{i}\right) \text { list } \\
& \text { norm-eff } i p c \tau \equiv \operatorname{map}\left(\lambda p c^{\prime} .\left(p c^{\prime},\left\lfloor\text { eff } f_{i} i\right\rfloor\right)\right)(\text { succs } i \tau p c) \text {. }
\end{aligned}
$$

The result is a list of edges in the control flow graph determined by succs, each of them marked with the result of $e f f_{i}$. As we use norm-eff only for the reachable instructions in the following, we can safely mark the successors as reachable with $\left.L_{-}\right\rfloor$.

We now have two functions, $a p p_{i}$ and norm-eff, describing normal execution in the bytecode verifier. The next section turns to exceptions.

4.7.2 Exceptions. Abstractly, exceptions merely add more edges to the control flow graph. In the JVM (Jinja as well as Java), these edges must all lead to the start of the exception handler that is relevant for the current instruction. Only at runtime is this relevant handler uniquely determined; statically, we must consider a number of handlers because the relevance of a handler depends on which exception was raised. The Invoke instruction, for instance, may raise a NullPointer exception or it may propagate an exception up that was thrown in the invoked method. For each of these cases a different handler might be relevant. As for the conditional branch instruction, the bytecode verifier simply checks all of them.

Thus, the first thing we need to do is to determine which handlers might be relevant for an instruction. Figure 26 shows this in three stages: relevant-class $i P C$ is True iff $i$ can raise an exceptions of class $C$, is-relevant-entry $P i$ pce is True iff entry $e$ in the exception table might match instruction $i$ at position $p c$, and finally, relevant-entries $P i p c x t$ is the list of exception table entries that are relevant for instruction $i$ at position $p c$.

For applicability in the exception case, we require that the class name mentioned in the exception handler is indeed a declared class, and that the stack is between $m x s$ and the number $d$ of entries the exception handler expects.

xcpt-app :: instr $\Rightarrow p c \Rightarrow t y_{i} \Rightarrow$ bool

xcpt-app i pc $(S T, L T) \equiv$

$\forall(f, t, C, h, d) \in \operatorname{set}$ (relevant-entries $P$ i pc $x t$ ). is-class $P C \wedge d \leq|S T| \wedge d<m x s$ 
The effect of instructions in the exception case is equally simple. Each edge leads to the start of the exception handler, and the local variables are unchanged. We cut down the operand stack to $d$ elements of the current stack, and push the exception object on top.

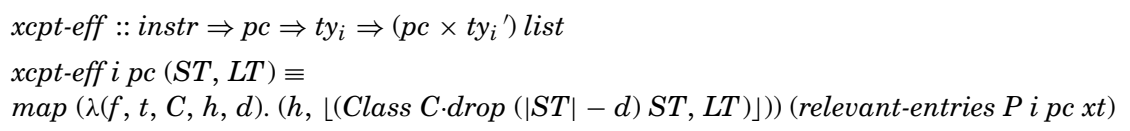

4.7.3 Combining Normal and Exceptional Execution. Combining the normal and exceptional case, we can now build the full effect function: If an instruction is unreachable, it has no outgoing edges; if it is reachable, the overall effect is simply an append of the normal and the exception case.

eff :: instr $\Rightarrow p c \Rightarrow t y_{i}{ }^{\prime} \Rightarrow\left(p c \times t y_{i}{ }^{\prime}\right)$ list

eff $i$ pc $t \equiv$ case $t$ of None $\Rightarrow[] \mid\lfloor\tau\rfloor \Rightarrow$ norm-eff $i$ pc $\tau$ @ xcpt-eff $i$ pc $\tau$

For applicability we have: An instruction is applicable if it is unreachable (then it can do no harm) or if it is applicable in the normal and in the exception case. Additionally, we require that the $p c$ does not leave the instruction sequence.

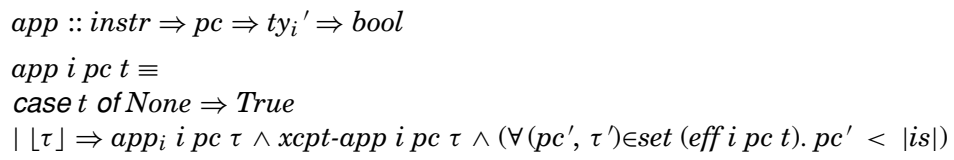

\subsection{Well-Typings}

Having defined the semilattice and the transfer function in Sections 4.6 and 4.7 , we show in this section how the parts are put together to get a definition of well-typings for the Jinja VM.

The abstract framework gives us a predicate wt-app-eff (Section 4.4) describing well-typings $\tau s:: t y_{i}{ }^{\prime}$ list as method types that fit an instruction sequence. To obtain type-safety and an executable bytecode verifier, we additionally require a start condition for instruction 0 (at method invocation) and some side conditions explained in the following.

The operational semantics of Invoke tells us the start condition at method invocation: The stack is empty, the first register contains the this-pointer (of type Class $C^{\prime}$ ), the next registers contain the parameters of the method, and the rest of the registers are reserved for local variables (which do not have a value yet). Note that the definitions are still in the context of a fixed method, as defined in Section 4.7, so $C^{\prime}$ is the class to be verified, $T s$ are the parameters, and $m x l_{0}$ the number of local variables (which is related to $m x l$ of Section 4.7 by $\left.m x l=1+|T s|+m x l_{0}\right)$. The $\leq^{\prime}$ is the semilattice order on $t y_{i}{ }^{\prime}$ of Section 4.6.

$w t$-start $\tau s \equiv P \vdash\left\lfloor\left([], O K\left(\right.\right.\right.$ Class $\left.C^{\prime}\right) \cdot m a p$ OK Ts @ replicate $\left.\left.m x l_{0} E r r\right)\right\rfloor \leq^{\prime} \tau s_{[0]}$

The method type $\tau s$ is a well-typing for a method if it satisfies $w t$-method. To define it, we instantiate $w t$-app-eff from the framework with $\lambda p c \tau$. app $i s_{[p c]} p c$ $\tau$ for the abstract $a p p$, and $\lambda p c \tau$. eff $i s_{[p c]} p c \tau$ for the abstract eff. 
wt-method $\tau s=$

$\left(i s \neq[] \wedge|\tau s|=m p c \wedge O K^{\prime}\right.$ set $\tau s \subseteq$ states $P m x s m x l \wedge$ wt-start $\tau s \wedge$ wt-app-eff $\left.\tau s\right)$

The states are the carrier set of the semilattice. Recall that the method type $\tau s$ does not contain the Err layer of the semilattice, hence, we take the image $O K$ ' set $\tau s$ of set $\tau s$ under $O K$. For well-typedness, wt-method also requires that the method contains at least one instruction and that the method type covers all instructions.

It is occasionally useful in the proofs, and also for the compiler to follow, to define the notion of an instruction being well-typed. This is just the matrix of the $\forall p<|\tau s|$ in $w t$-app-eff (c.f. Section 4.4). Since it will be used outside the fixed method context of this section, it has more parameters.

$P, T_{r}, m x s, m p c, x t \vdash i, p c:: \tau s \equiv$

app i pc $\tau s_{[p c]} \wedge\left(\forall\left(p c^{\prime}, \tau\right) \in \operatorname{set}\left(\right.\right.$ eff $\left.\left.i p c \tau s_{[p c]}\right) . P \vdash \tau^{\prime} \leq^{\prime} \tau s_{\left[p c^{\prime}\right]}\right)$

The notation $P, T_{r}, m x s, m p c, x t \vdash i, p c:: \tau s$ is read as: In the context of program $P$, return type $T_{r}$, maximum stack size $m x s$, number of instructions $m p c$ and exception table $x t$, the instruction $i$ at position $p c$ is well-typed w.r.t. method type $\tau s:: y_{i}{ }^{\prime}$ list.

It remains to lift well-typings from methods to programs. Well-typings of programs are functions $\Phi:: t y_{P}$ with

$$
\text { types } t y_{P}=\text { cname } \Rightarrow \text { mname } \Rightarrow t y_{i}{ }^{\prime} \text { list }
$$

These functions return a well-typing for each class and method in the program. A Jinja VM program is well-formed according to $\Phi$ if each method body is welltyped. At this point, the full parameter list of wt-method becomes visible.

$w f-j v m-\operatorname{prog}_{\Phi} \equiv$

$w f-p r o g\left(\lambda P C\left(M, T s, T_{r}, m x s, m x l_{0}, i s, x t\right)\right.$. wt-method $P C$ Ts $T_{r} m x s m x l_{0}$ is $\left.x t(\Phi C M)\right)$

$w f$-jum-prog $P \equiv \exists \Phi$. wf-jum-prog $\Phi$

\subsection{An Executable Bytecode Verifier}

In Section 4.8 we defined well-typings for the Jinja VM. This section shows how to instantiate the type inference algorithm of Section 4.5 to get an executable bytecode verifier for Jinja.

With the semilattice as defined in Section 4.6 and the transfer function of Section 4.7, and still within the same method context as for wt-method, we only need to provide the correct start value to Kildall's algorithm to get an executable BV:

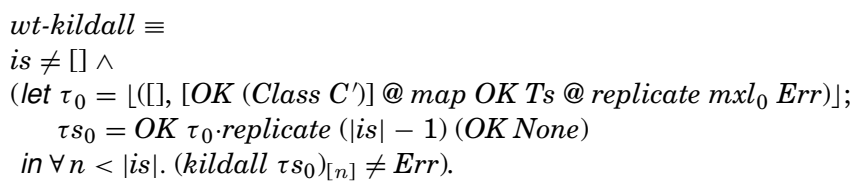

Position 0 in $\tau s_{0}$ is the same as the start value in $w t$-start. Since we know nothing yet about the positions greater than 0 , we fill in the bottom element OK None for these. 
Lifting to full programs and filling in the method context is the same as for wt-method:

$w f$-jvm- $\operatorname{prog}_{k} P \equiv$

wf-prog $\left(\lambda P C^{\prime}\left(M, T s, T_{r}, m x s, m x l_{0}\right.\right.$, is, xt). wt-kildall $P C^{\prime} T s T_{r} m x s m x l_{0}$ is $\left.x t\right) P$.

This definition only gives us a working BV if step meets the conditions of Theorem 4.11. We have shown that the transfer function step, built from app and eff as described in Sections 4.4 and 4.7, is monotone, bounded, and type preserving (w.r.t. states and $|i s|$ ). Albeit large (a case distinction over the instruction set), the proof that step is monotone and type preserving is easy and mostly automatic. That step is bounded is checked explicitly by the app component of step.

Using Theorem 4.11, we have thus proved the following:

THEOREM 4.14. The executable BV is sound and recognises all well-typed programs:

$$
w f-j v m-\operatorname{prog}_{k} P=w f \text {-jum-prog } P
$$

\subsection{Type-Safety}

This section is about the type-safety of the aforementioned well-typing specification. The type-safety theorem states that the bytecode verifier is correct and that it guarantees safe execution. If the bytecode verifier succeeds and we start the program $P$ in its canonical start state (Section 3.3), the defensive Jinja VM will never return a type error. With Theorem 3.1, this implies that the checks of the defensive machine are redundant and the aggressive machine can be used safely instead.

Theorem 4.15. If $C$ is a class in $P$ with a method $M$, formally $P \vdash C$ sees $M:[] \rightarrow T=b$ in $C$, and if wf-jum-prog $P$, then

$$
P \vdash \text { Normal (start-state } P C M \text { ) } \stackrel{\prime \text { djvm }}{\longrightarrow} \sigma^{\prime} \Longrightarrow \sigma^{\prime} \neq \text { TypeError. }
$$

To prove this theorem, we set out from a program $P$ for which the bytecode verifier returns true, that is, for which there is a $\Phi$ such that $w f-j v m-p r o g_{\Phi}$ $P$ holds. The proof builds on the observation that all runtime states $\sigma$ that conform to the types in $\Phi$ are type-safe. For $\sigma$ conforms to $\Phi$, we write $P, \Phi \vdash \sigma \sqrt{ }$. For $P, \Phi \vdash \sigma \sqrt{ }$ to be true, the following must hold: if in state $\sigma$ execution is at position $p c$ of method $M$ in $C$, then the state type ( $\Phi C M)_{[p c]}$ must be of the form $\lfloor\tau\rfloor$, and for every value $v$ on the stack or in the register set, the type of $v$ must be a subtype of the corresponding entry in its static counterpart $\tau$. This is the essence of the conformance relation. To show that it is invariant during execution, it needs to be strengthened. We show the complete formal definition of conformance in the following, but to avoid getting bogged down in detail we continue to sketch the general proof outline first. For this strong conformance we have shown that it is invariant during execution if the program is well-typed.

LEMMA 4.16. If $w$ f-jum- $\operatorname{prog}_{\Phi} P$ and $P \vdash \sigma \stackrel{\text { jvm }}{\longrightarrow} \sigma^{\prime}$ and $P, \Phi \vdash \sigma \sqrt{ }$, then $P, \Phi \vdash \sigma^{\prime} \sqrt{ }$. 
The proof is by induction on the length of the execution and then by case distinction on the instruction set. For each instruction, we conclude from the conformance of $\sigma$ together with the app part of $w f$-jum-prog that all assumptions of the operational semantics are met (such as "the stack is not empty"). Then we execute the instruction and observe that the new state $\sigma^{\prime}$ conforms to the corresponding $\tau^{\prime}$ in eff pc $\tau$. This invariance lemma corresponds to subject reduction in a traditional small step semantics.

Lemma 4.16 is still not sufficient for type-safety, though; it might be the case that start $P C M$ does not conform to $\Phi$. We have shown that this is not so.

Lemma 4.17. If $w f$-jum- $\operatorname{prog}_{\Phi} P$ and $P \vdash C$ sees $M$ : [] $\rightarrow T=m$ in $C$, then $P, \Phi \vdash$ start-state P $C M \sqrt{ }$.

Lemmas 4.16 and 4.17 together say that all states that occur in any execution of program $P$ conform to $\Phi$ if we start $P$ in the canonical way.

The last step in the proof of Theorem 4.15 is Lemma 4.18. It corresponds to progress in a traditional small step semantics.

LEMMA 4.18. An execution step started in a conformant state cannot produce a type error in well-typed programs:

$$
\text { If } w \text {-jum-prog } \Phi \text { } P \text { and } P, \Phi \vdash \sigma \sqrt{ } \text { then } \text { exec }_{d} P \sigma \neq \text { TypeError. }
$$

The proof of Lemma 4.18 is by case distinction on the current instruction in $\sigma$. Similar to the proof of Lemma 4.16, the conformance relation together with the app part of $w f$-jum-prog ensures check-instr in exec $c_{d}$ returns true. Because we know that all states during execution conform, we can conclude as in Theorem 4.15: There will be no type errors in well-typed programs.

We now show the formal definition of the conformance relation between dynamic Jinja VM states and static states (types) in the Jinja BV.

For the proof of the invariance lemma (Lemma 4.16) to go through, the intuitive notion of conformance we have just given is not sufficient; the formal conformance relation $P, \Phi \vdash \sigma \sqrt{ }$ is stronger. It describes in detail the states that can occur during execution, the form of the heap, and the form of the method invocation stack.

We begin by lifting the single value conformance $P, h \vdash v: \leq T$ of Section 2.9 to stack and local variables. For the stack, this is just the pointwise lifting of $: \leq$ to lists. As usual, we write $P, h \vdash v s[: \leq] S T$ for this. For the local variables, we first need to treat the Err level:

$P, h \vdash v: \leq \top E \equiv$ case $E$ of $E r r \Rightarrow$ True $\mid O K T \Rightarrow P, h \vdash v: \leq T$.

Lifting to lists $P, h \vdash v s\left[: \leq_{\top}\right] L T$ is then canonical again.

A call frame conforms if its stack and register set conform and if the program counter lies inside the instruction list.

conf-f $P h(S T, L T)$ is (stk, loc, $C, M, p c) \equiv$

$P, h \vdash \operatorname{stk}[: \leq] S T \wedge P, h \vdash \operatorname{loc}[: \leq \mathrm{T}] L T \wedge p c<|i s|$

This is still not enough. For proving the Return case, we also need information about the structure of the call frame stack. The predicate conf-fs which follows describes the structure of the call frame stack beneath the topmost frame. The 
parameters $M_{0}, n_{0}$, and $T_{0}$ are the name, number of parameters, and return type of the method in the topmost frame.

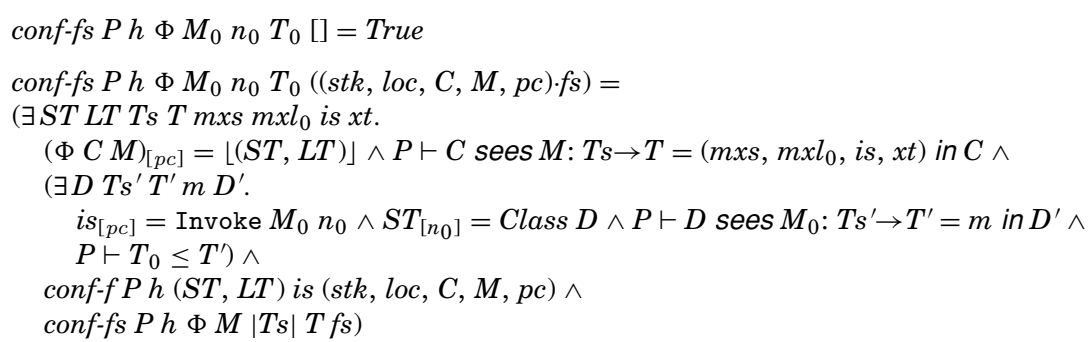

In the preceeding definition, a list of call frames conforms if it is empty. If it is not empty, then for the head frame, the state type ( $\Phi C M)_{[p c]}$ for the current instruction must denote a reachable instruction, the call frame must belong to a defined method, it must be halted at the Invoke instruction which created the call frame above (this is not easily expressed without access to something like a call history, but it is enough to demand that the $M$ and $n$ in Invoke $M n$ are $M_{0}$ and $n_{0}$, and that the return type of a lookup on the class $D$ in $S T_{\left[n_{0}\right]}$ conforms to $T_{0}$, the return type the frame above expects), and finally, the current frame and the rest of the call frame stack must conform.

The following is the top level conformance relation between a state and a program type. The first two cases are trivial, the third case requires a conformant heap $(P \vdash h \sqrt{ })$, and contains special handling for the topmost call frame and delegates the rest to conf- $f s$. The topmost frame is special because it does not need to be halted at an Invoke instruction. The topmost frame must conform and the current state type must denote a reachable instruction. The method lookup provides conf- $f$ and conf- $f s$ with the required parameters.

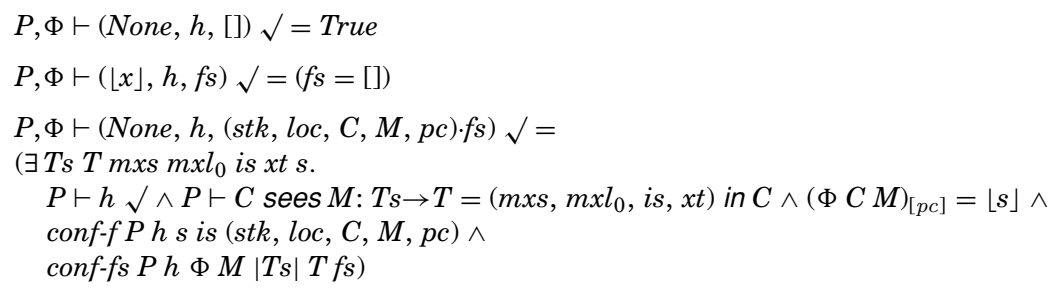

Figure 27 is a snapshot of the Jinja VM state in the middle of a typical program execution. On the left there is the Jinja VM with its frame stack and heap, and on the right there are the method types the BV predicted for this program. The program declarations appear on the lower right side in the static part.

The state on the left in Figure 27 conforms to the static type information on the right: All objects in the heap conform because the values of the field $F$ (declared in class $B$ ) are all of type Class $A$ (Null is of type Class $A$, and the address $A d d r 0$ points to an object of Class $B$, which is a subclass of Class $A$ ). All frames but the topmost one are halted at the Invoke instruction that created the next frame. The dynamic operand stacks conform to the static ones because their length is the same and all values have conforming type. The topmost 


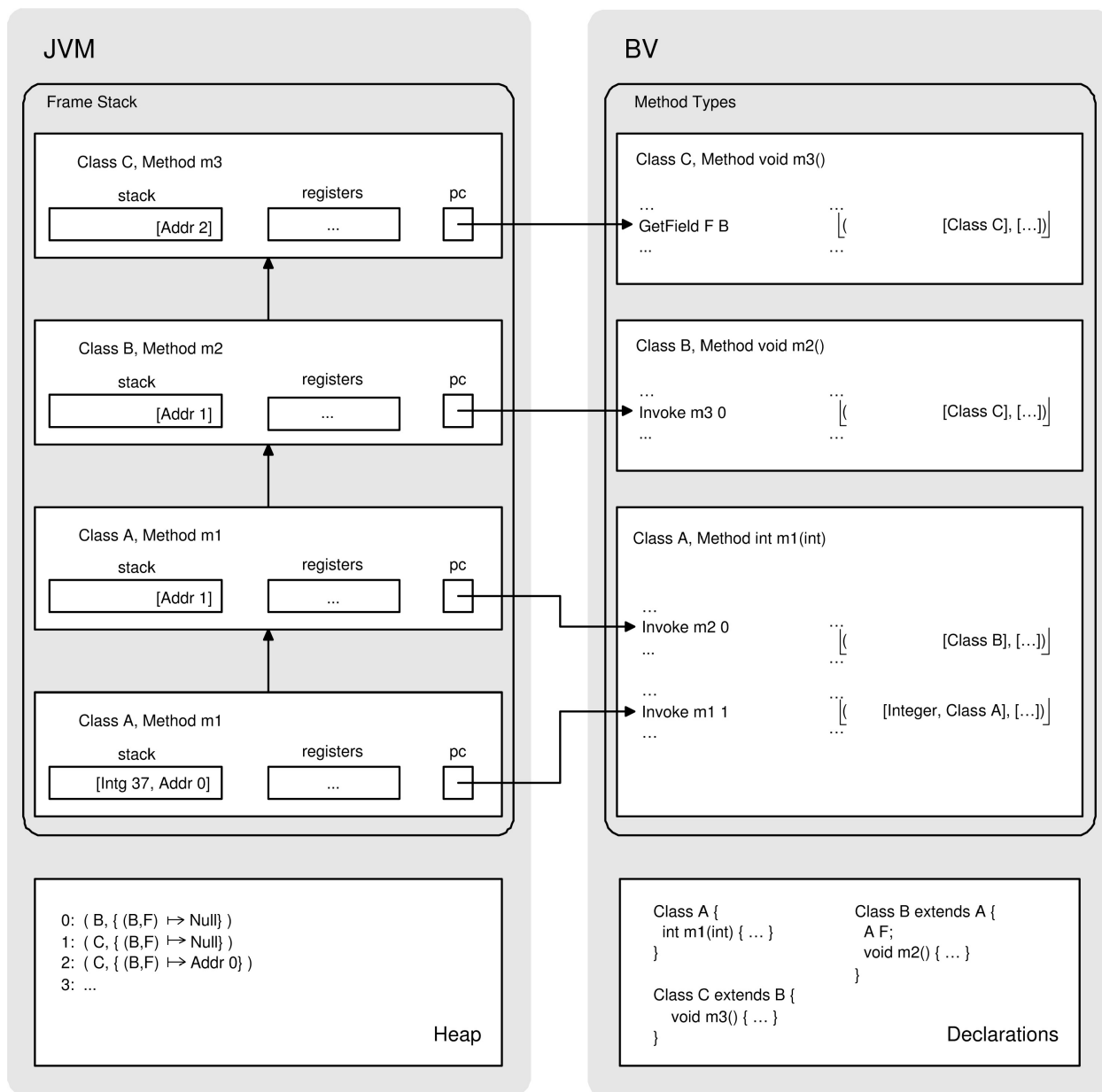

Fig. 27. Jinja VM execution snapshot.

frame conforms as well because its $p c$ points to a valid instruction (Getfield $F$ $B$ ), and the value on the dynamic operand stack is an address that points to an object of class $C$.

\subsection{Related Work}

The bytecode verifier presented here is a simplified version of the one by Klein [2003], which in turn built on the work by Nipkow [2001] and Pusch [1999]. Most closely related to these is the work by Barthe and Dufay [2004] who use the Coq system for a formalisation of the JavaCard virtual machine and its BV. They formalise the full JavaCard bytecode language, but with a simplified treatment of some features like bytecode subroutines.

The ASM formalisation of Stärk et al. [2001] contains almost all of the Java BV's features and is executable. It does not use mechanical support or checking for its proofs. 
Paper formalisations of Java bytecode verification begin with Stata and Abadi [1998] who give a first formal type system for the JVM. Freund and Mitchell [2003] and Freund [2000] build on this type system and extend it to a substantial subset of the JVM.

Working towards a verified implementation in SPECWARE, Coglio et al. [2000] have specified and analysed large portions of the bytecode verifier. Goldberg [1998] rephrases and generalises the overly concrete description of the BV given in the JVM specification [Lindholm and Yellin 1999] as an instance of a generic data flow framework. Qian [2000] also proves the correctness of an algorithm for turning his type checking rules into a data flow analyser. However, his algorithm is still quite abstract. The formulation of the bytecode verification algorithm as a direct instance of an abstract data flow framework first appears in the work by Nipkow [2001] and is refined by Klein and Nipkow [2003] and Klein [2003].

There is an interesting variant of type inference for the JVM, namely, lightweight bytecode verification [Rose and Rose 1998; Rose 2002, 2003]. It uses a certificate to reduce time and space usage during type inference. For space reasons, we have omitted it here, but lightweight bytecode verification fits in nicely with our formalisation of the VM type system [Klein and Nipkow 2001]. Moreover, it can be formulated as an inference algorithm in the same abstract framework that we use for Kildall's data flow analyser [Klein 2003]. Other variations of lightweight bytecode verification algorithms are surveyed by Leroy [2003].

There is a large body of literature on bytecode verification that concentrates on special language features such as subroutines [Coglio 2004; Klein and Wildmoser 2003], object initialisation, dynamic class loading, thread monitors, and access control. There are also a number of more or less radically different approaches to type inferences like model checking, reducing the BV to Haskell type inference, and using ML-style polymorphism. We refer to the overview articles for these.

\section{COMPILER}

Our compiler operates in two stages: First, it replaces variable names in expressions by indices (Section 5.2), then it generates the actual JVM code (Section 5.3). Once we have lifted compilation of expressions to the level of programs (Section 5.4), we show that both compiler stages preserve the big step semantics (Sections 5.5 and 5.6). Finally, we show that not only the semantics, but also the well-typedness of expressions and well-formedness of programs is preserved by compilation, that is, that the compiler produces well-typed JVM code (Sections 5.8 and 5.9).

\subsection{Intermediate Language}

The intermediate language of expressions is called expr $r_{1}$ and is identical to expr except that all variable names are replaced by natural numbers, save for field access and field assignment. To avoid duplication, in Isabelle/HOL we have defined one parameterised data type 'a exp, and expr and expr ${ }_{1}$ are 


$$
\begin{aligned}
& \frac{l s_{[i]}=v \quad i<|l s|}{P \vdash_{1}\langle\operatorname{Var} i,(h, l s)\rangle \Rightarrow\langle\operatorname{Val} v,(h, l s)\rangle} \\
& \frac{P \vdash_{1}\left\langle e, s_{0}\right\rangle \Rightarrow\langle\operatorname{Val} v,(h, l s)\rangle \quad i<|l s| \quad l s^{\prime}=l s[i:=v]}{P \vdash_{1}\left\langle i:=e, s_{0}\right\rangle \Rightarrow\left\langle\text { unit },\left(h, l s^{\prime}\right)\right\rangle} \\
& P \vdash_{1}\left\langle e_{1}, s_{0}\right\rangle \Rightarrow\left\langle\text { Throw } a,\left(h_{1}, l s_{1}\right)\right\rangle \quad h_{1} a=\lfloor(D, f s)\rfloor \\
& \frac{P \vdash D \preceq{ }^{*} C \quad i<\left|l s_{1}\right| \quad P \vdash_{1}\left\langle e_{2},\left(h_{1}, l s_{1}[i:=A d d r a]\right)\right\rangle \Rightarrow\left\langle e_{2}{ }^{\prime},\left(h_{2}, l s_{2}\right)\right\rangle}{P \vdash_{1}\left\langle\operatorname{try} e_{1} \operatorname{catch}(C i) e_{2}, s_{0}\right\rangle \Rightarrow\left\langle e_{2}{ }^{\prime},\left(h_{2}, l s_{2}\right)\right\rangle} \\
& \frac{P \vdash_{1}\left\langle e, s_{0}\right\rangle \Rightarrow\left\langle e^{\prime}, s_{1}\right\rangle}{P \vdash_{1}\left\langle\{i: T ; e\}, s_{0}\right\rangle \Rightarrow\left\langle e^{\prime}, s_{1}\right\rangle} \\
& P \vdash_{1}\left\langle e, s_{0}\right\rangle \Rightarrow\left\langle a d d r a, s_{1}\right\rangle \quad P \vdash_{1}\left\langle e s, s_{1}\right\rangle[\Rightarrow]\left\langle\text { map Val vs, }\left(h_{2}, l s_{2}\right)\right\rangle \\
& h_{2} a=\lfloor(C, f s)\rfloor \quad P \vdash C \text { sees } M: T s \rightarrow T=b o d y \text { in } D \\
& |v s|=|T s| \quad l s_{2}{ }^{\prime}=\text { Addr a.vs @ replicate (max-vars body) arbitrary } \\
& \begin{array}{c}
P \vdash_{1}\left\langle\operatorname{body},\left(h_{2}, l s_{2}{ }^{\prime}\right)\right\rangle \Rightarrow\left\langle e^{\prime},\left(h_{3}, l s_{3}\right)\right\rangle \\
P \vdash_{1}\left\langle e \cdot M(e s), s_{0}\right\rangle \Rightarrow\left\langle e^{\prime}\left(h_{3}, l s_{2}\right)\right\rangle
\end{array}
\end{aligned}
$$

Fig. 28. Evaluation rules for expr 1 differing from those for expr.

abbreviations for the instances vname exp and nat exp. The type of $J_{1}$ programs is defined by

$$
\text { types } J_{1} \text {-prog }=\operatorname{expr}_{1} \text { prog. }
$$

In contrast to $J$-prog, methods no longer need parameter names because they have been replaced by numbers.

5.1.1 Big Step Semantics. The motivation for our choice of intermediate language is that its semantics is based on almost the same state space as the JVM: Local variables are no longer stored in a map but in a fixed length list of values, that is, in an array. Its big step semantics is of the form $P \vdash_{1}\langle e, s\rangle \Rightarrow$ $\left\langle e^{\prime}, s\right\rangle$, where $P:: J_{1}$-prog, e, $e^{\prime}:: \operatorname{expr}_{1}$, and $s, s^{\prime}::$ heap $\times$ val list.

Most of the evaluation rules for expr 1 are the same as the ones for expr. The ones that differ are shown in Figure 28. The modifications in the rules for variable access, variable assignment, and exception catching simply reflect the difference between a map and an array. Note that the rules are defensive in that they require the index to be within the bounds of the array. The rule for blocks expresses that all local variables are preallocated and blocks are irrelevant for the semantics. However, they are not irrelevant for the type system because of the types of local variables.

The method call rule has changed because the state in which the body is evaluated needs to be initialized with an array $\left(l s_{2}\right)$. The initial section of that array contains the address of the object and the parameters, and the remainder is initalized with an arbitrary value just as in the semantics of Invoke in the JVM (see Section 3.2). To find out how many local variables are needed, max-vars (Figure 30) computes the maximal depth of nested local variables. However, the depth may say nothing about the actual indices, for example, in $\left\{29: T_{0} ;\left\{17: T_{1} ; e\right\}\right\}$. But the layout of $l s_{2}{ }^{\prime}$ assumes the two are related: All indices in body must be below $1+$ size vs + max-vars body. This assumption is justified because, as we will see later, stage 1 of the compiler produces intermediate 


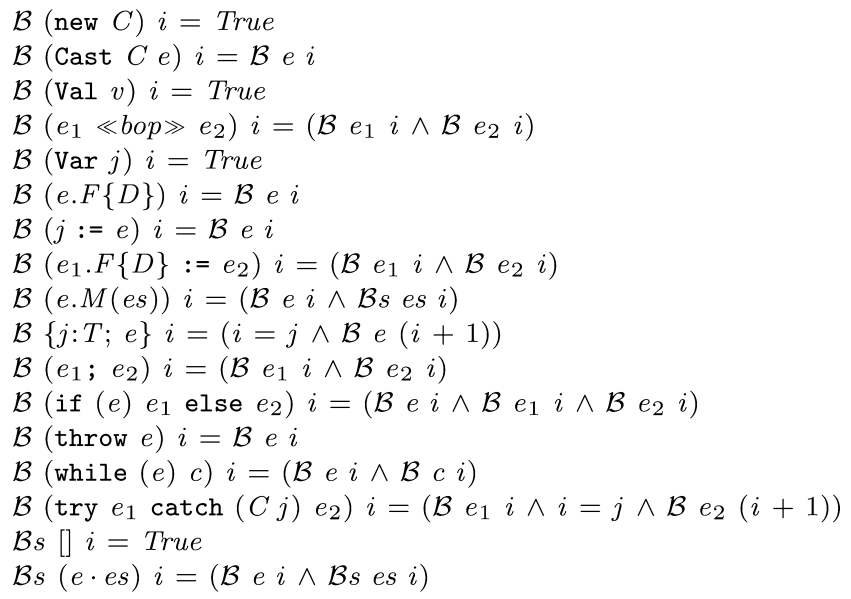

Fig. 29. Definition of $\mathcal{B}$.

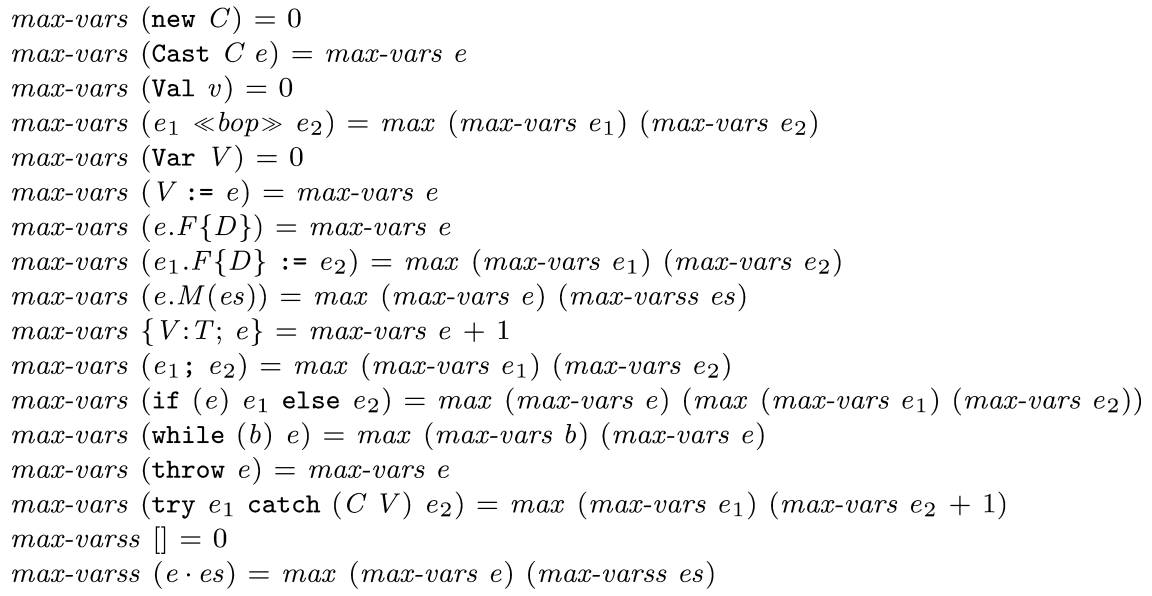

Fig. 30. Definition of max-vars.

expressions with the following property: Starting from some given index, say 4, indices increase by 1 for each nested block. For example, $\left\{5: T_{0} ;\left\{6: T_{1} ; e_{1}\right\} ;\left\{6: T_{2} ;\right.\right.$ $\left.\left.e_{2}\right\}\right\}$ is fine, but $\left\{6: T_{1} ;\left\{5: T_{0} ; e\right\}\right\},\left\{5: T_{0} ;\left\{7: T_{2} ; e\right\}\right\}$, and $\left\{6: T_{1} ;\left\{7: T_{0} ; e\right\}\right\}$ are not. This property is formalized by $\mathcal{B}$ in Figure 29 and could be called an inverse de Bruijn numbering scheme.

5.1.2 Type System. Again we replace a map by a list: In the judgment $P, E$ $\vdash_{1} e:: T$, the environment $E$ is now of type ty list (and $P:: J_{1}-p r o g$ ). Most rules for this type system are identical to the ones for expr. The ones that have changed are shown in Figure 31. They also rely on the inverse de Bruijn numbering scheme by ignoring the $i$ in $\{i: T ; e\}$.

5.1.3 Well-Formedness. Well-formedness of $J_{1}$ programs is essentially well-formedness of Jinja programs (Section 2.8) where the conditions on the 


$$
\begin{gathered}
\frac{E_{[i]}=T \quad i<|E|}{P, E \vdash_{1} \operatorname{Var} i:: T} \\
\frac{E_{[i]}=T \quad i<|E| \quad P, E \vdash_{1} e:: T^{\prime} \quad P \vdash T^{\prime} \leq T}{P, E \vdash_{1} i:=e:: \text { Void }} \\
\frac{\text { is-type } P T \quad P, E @[T] \vdash_{1} e:: T^{\prime}}{P, E \vdash_{1}\{i: T ; e\}:: T^{\prime}} \\
\frac{P, E \vdash_{1} e_{1}:: T \quad[\text { Class } C] \vdash_{1} e_{2}:: T}{P, E \text { is-class } P C} \\
P \vdash_{1} \text { try } e_{1} \text { catch }(C i) e_{2}:: T
\end{gathered}
$$

Fig. 31. Typing rules for expr 1 differing from those for expr.

parameter names have been dropped and inverse de Bruijn numbering is required:

$w f-J_{1}-m \operatorname{decl} P C(M, T s, T, b o d y) \equiv$

$\left(\exists T^{\prime} . P\right.$, Class $C \cdot T s \vdash_{1}$ body :: $\left.T^{\prime} \wedge P \vdash T^{\prime} \leq T\right) \wedge \mathcal{D}$ body $\lfloor\{. .|T s|\}\rfloor \wedge \mathcal{B}$ body $(|T s|+1)$

$w f-J_{1}-$ prog $\equiv w f-p r o g w f-J_{1}-m d e c l$

In the definition of $\mathcal{D}$ (Section 2.7) we pretended that it operates on expr, but in reality it is defined on the polymorphic type ' $a$ exp and hence works on $\operatorname{expr}_{1}$ as well.

\subsection{Stage 1: Names to Indices}

The translation from expr to expr $r_{1}$ is parametrised by the list of variables declared on the path from the root of the expression to the current subexpression. The index of a variable is the position of its rightmost occurrence in that list: In $[A, B, A, C, D], A$ has index $2, B$ index 1 , and $C$ index 3 . The formal definition index []$y=0$

index $(x \cdot x s) y=($ if $x=y$ then if $x \in$ set $x$ s then index $x s y+1$ else 0 else index $x s y+1)$

reveals that the index of an element not in the list is the length of the list. Function compE $E_{1}::$ vname list $\Rightarrow$ expr $\Rightarrow \operatorname{expr}_{1}$ (Figure 32) traverses an expression and translates variable names via index.

\subsection{Stage 2: Code Generation}

Code generation comprises the generation of an instruction list and an exception table. Generating the instruction list (function $\operatorname{comp}_{2}::$ expr $_{1} \Rightarrow$ instr list in Figure 33) is straightforward since we are already on the level of indices rather than names. The only case that may need some explanation is try-catch. We compile the code as suggested in the official JVM specification [Lindholm and Yellin 1999]: first, the try block, then a jump over the rest to the end, then (and this is the entry point for the exception handler) the exception reference is stored in the local variable, and finally, the catch block. Remember that int is the injection from naturals (produced by the length function) into integers (required by branch instructions).

Producing the code is simplified because branch instructions are relative. Hence, the compiler does not need to compute an absolute address. The 


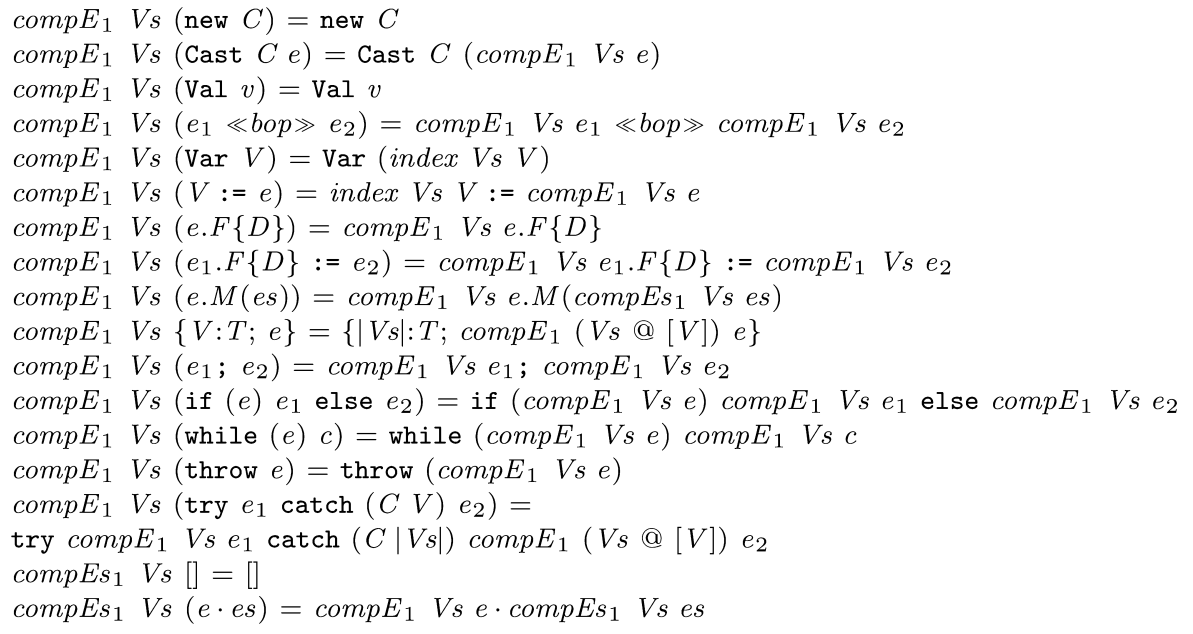

Fig. 32. Definition of comp $E_{1}$.

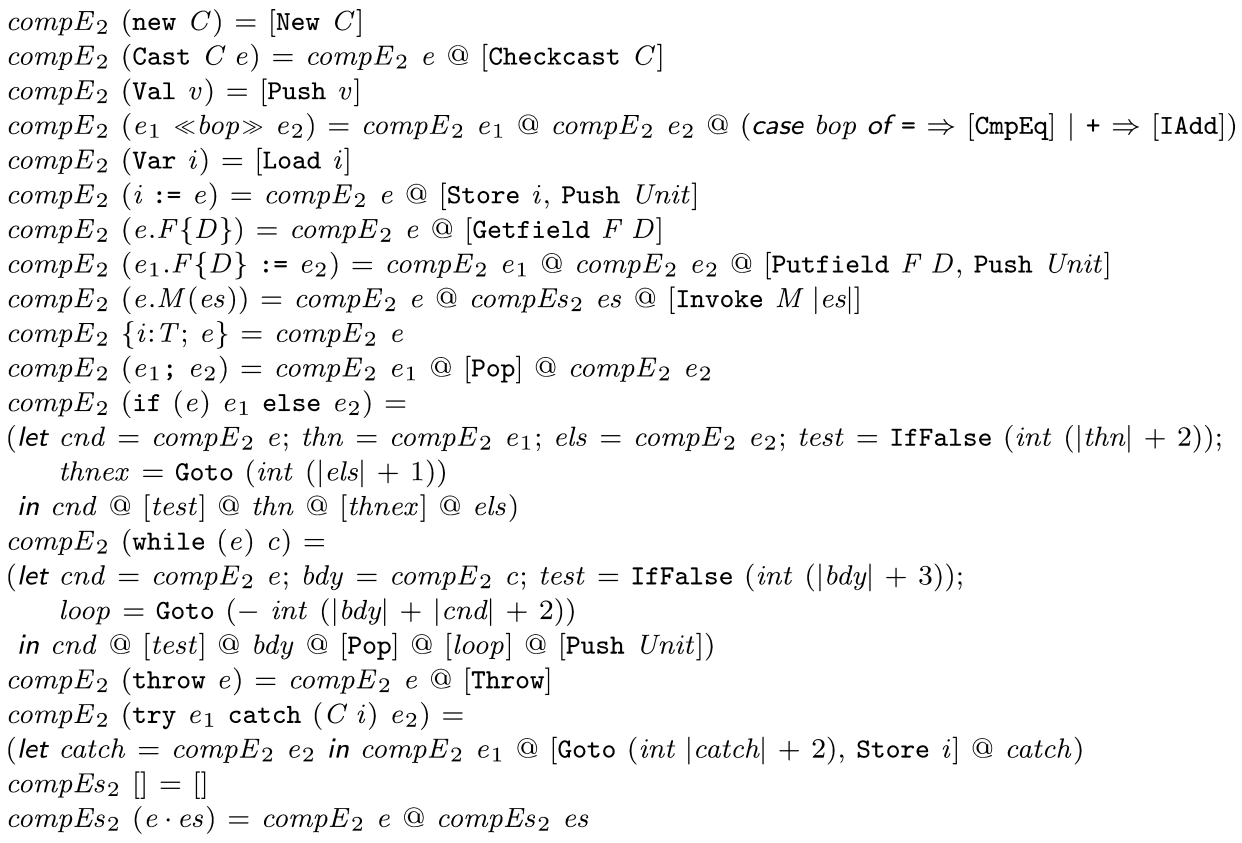

Fig. 33. Definition of $c o m p E_{2}$.

exception table, however, contains absolute addresses. Hence, its generation (function $\operatorname{compxE} E_{2}:: \operatorname{expr}_{1} \Rightarrow p c \Rightarrow$ nat $\Rightarrow$ ex-table in Figure 34) requires the current program counter as a parameter. The final parameter is the current size of the stack. Function compx $E_{2}$ traverses the expression top-down and left-to-right, collecting the handler information from all the try-catch constructs it encounters while keeping track of the current program counter (by adding the size of the compiled code to the left to it) and the current stack 


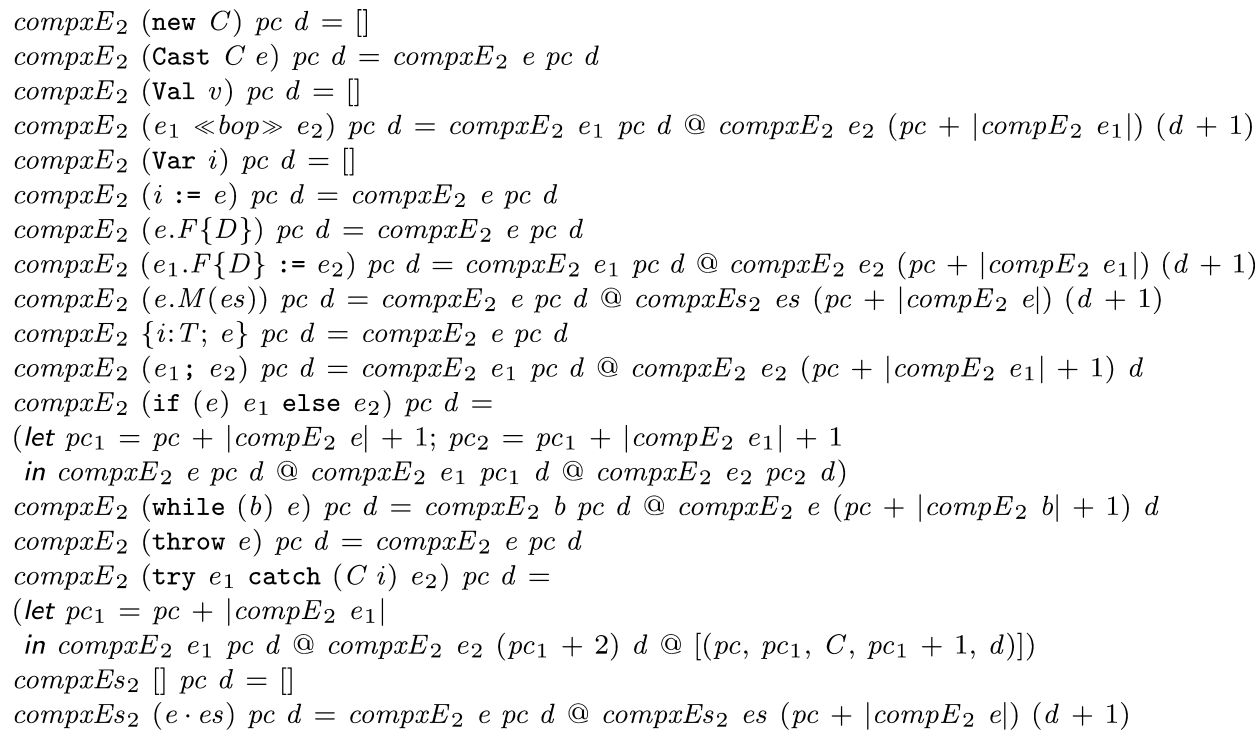

Fig. 34. Definition of compx $E_{2}$.

size (by incrementing it suitably). Each try-catch generates one entry in the exception table. It contains the stack size $d$ before execution of the try-catch; this is the stack size we must return to when an exception is encountered and execution continues with the catch block. The precise addresses in this exception table entry are, of course, implicitly determined by the layout of the code produced by $\operatorname{comp} E_{2}$. It is crucial that this entry is placed behind the exception tables belonging to the try and catch blocks: Because the JVM searches exception tables from the left, it will only find this entry if none of the more directly enclosing handlers match. At least this is the intuition. The proof follows.

\subsection{Program Compilation}

Lifting expression compilation to the level of programs is easy: Simply replace all method bodies by their compiled version. This is defined generically as

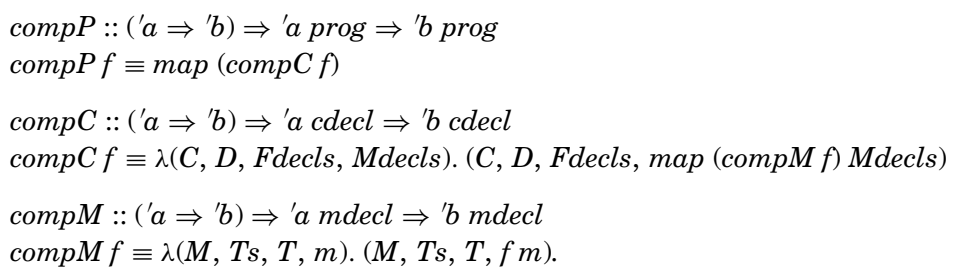

Now we can lift the two compiler stages from expressions to programs:

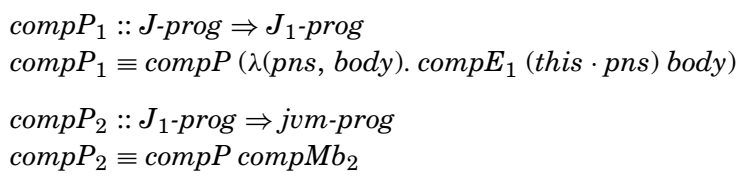




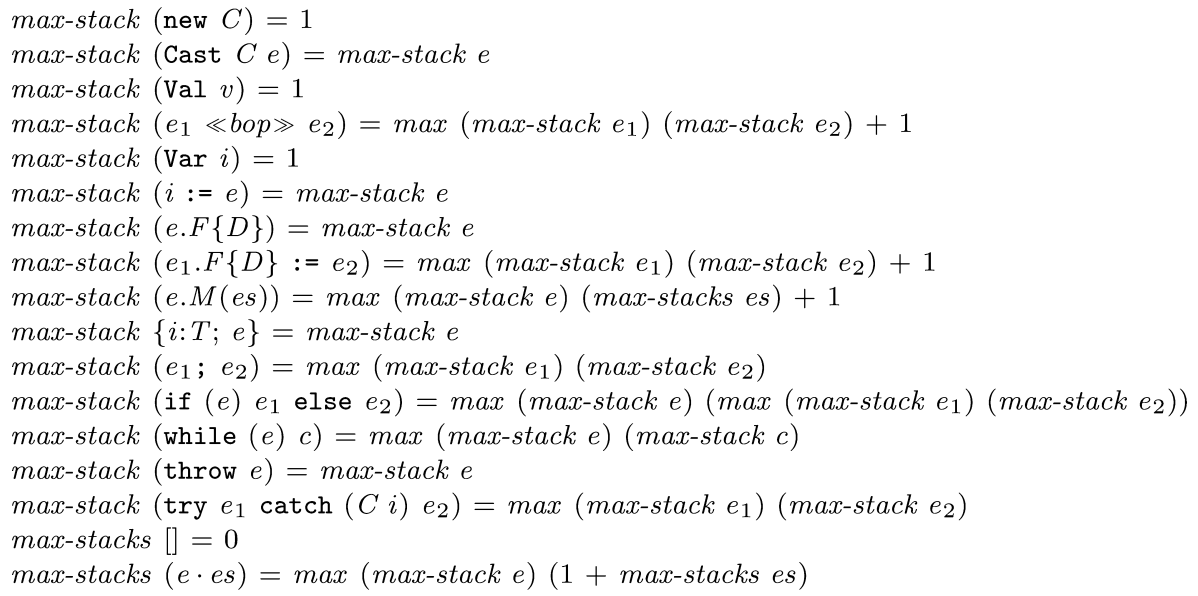

Fig. 35. Definition of max-stack.

$\operatorname{compMb} b_{2} \equiv$

$\lambda$ body. letins =comp $E_{2}$ body $@\left[\right.$ Return]; $x t=\operatorname{compx} E_{2}$ body 00

in (max-stack body, max-vars body, ins, $x t$ ).

Function comp $P_{1}$ compiles each method body in the context of this and uses its parameter names as the only local variables. Function $\operatorname{comp} P_{2}$ compiles each method body into the required 4-tuple of maximal stack size (Figure 35), the number of local variables, the instructions, and the exception table.

The main compiler $J 2 J V M$ is simply the composition of $c o m p P_{1}$ with compP $P_{2}$.

\subsection{Correctness of Stage 1}

Although the translation from names to indices appears straightforward, formulating its correctness is already a bit involved. We want to prove preservation of the semantics, that is, that an evaluation $P \vdash\langle e,(h, l)\rangle \Rightarrow\left\langle e^{\prime},\left(h^{\prime}, l^{\prime}\right)\right\rangle$ implies some evaluation $\operatorname{comp}_{1} P \vdash_{1}\left\langle\operatorname{comp}_{1} V s e,(h, l s)\right\rangle \Rightarrow\left\langle c o m p E_{1} V s e^{\prime},\left(h^{\prime}, l s^{\prime}\right)\right\rangle$. It is plausible that set $V s$ should be a superset of $f v e$ and that $[V s[\mapsto] l s]$ should be related to $l$. Equating them does not work because variables in $V s$ that have not yet been assigned to will not be in dom $l$-uninitialised variables again complicate matters. Hence, we have to settle for the weaker $l \subseteq_{m}[V s[\mapsto] l s]$ where

$m_{1} \subseteq_{m} m_{2} \equiv \forall a \in d o m m_{1} \cdot m_{1} a=m_{2} a$

is defind for arbitrary maps. This relationship should be preserved, that is, $l^{\prime}$ $\subseteq_{m}[V s[\mapsto] l s]$ should hold.

We are now ready for the main theorem.

Theorem 5.1. If wwf-J-prog $P$ and $P \vdash\langle e,(h, l)\rangle \Rightarrow\left\langle e^{\prime},\left(h^{\prime}, l^{\prime}\right)\right\rangle$ and fve $\subseteq$ set $V s$ and $l \subseteq_{m}[V s[\mapsto] l s]$ and $|V s|+$ max-vars $e \leq|l s|$ then $\exists l s^{\prime} . \operatorname{comp}_{1} P \vdash_{1}$ $\left\langle\operatorname{compE}_{1} V s e,(h, l s)\right\rangle \Rightarrow\left\langle f i n_{1} e^{\prime},\left(h^{\prime}, l s^{\prime}\right)\right\rangle \wedge l^{\prime} \subseteq_{m}\left[V s[\mapsto] l s^{\prime}\right]$.

First we examine the additional premises. Weak well-formedness is necessary for method calls: In order to apply the induction hypothesis arising from the evaluation of the method body, we need to establish an instance of $f v e \subseteq$ set $V s$, 
namely, that the only free variables in the body are this and the parameters. The premise $|V s|+$ max-vars $e \leq|l s|$ ensures that $l s$ is large enough to hold all local variables needed during the evaluation of $e$. In the conclusion, comp $E_{1} V s$ $e^{\prime}$ has been replaced by $f i n_{1} e^{\prime}$, where $f n_{1}:: \operatorname{expr} \Rightarrow \exp _{1}$ is the "identity" on final expressions: $f i n_{1}($ Val $v)=$ Val $v$ and $f i n_{1}($ Throw $a)=$ Throw $a$. This simplifies the proposition and the proof because it removes the pointless $V s$.

Proof. The proof is by induction on $P \vdash\langle e,(h, l)\rangle \Rightarrow\left\langle e^{\prime},\left(h^{\prime}, l^{\prime}\right)\right\rangle$. The only nontrivial cases are blocks and try-catch. We discuss the block case, as it isolates the main issue, which is local variables. Let $e$ be $\left\{V: T ; e_{0}\right\}$. From the assumptions $f v e \subseteq$ set $V s, l \subseteq_{m}[V s[\mapsto] l s]$ and $|V s|+$ max-vars $e \leq|l s|$, the preconditions for the induction hypothesis follow and we obtain an $l s^{\prime}$ such that compP $P_{1} P \vdash_{1}$ $\left\langle c o m p E_{1} V s_{0} e_{0},(h, l s)\right\rangle \Rightarrow\left\langle f i n_{1} e^{\prime},\left(h^{\prime}, l s^{\prime}\right)\right\rangle(1)$ and $l^{\prime} \subseteq_{m}\left[V s_{0}[\mapsto] l s\right](2)$, where $V s_{0}$ is $V s @[V]$. This $l s^{\prime}$ is also the witness for our goal. Its first conjunct comp $P_{1}$ $P \vdash_{1}\left\langle c o m p E_{1} V s e,(h, l s)\right\rangle \Rightarrow\left\langle f i n_{1} e^{\prime},\left(h^{\prime}, l s^{\prime}\right)\right\rangle$ follows directly from (1) by the rule for blocks. The second conjunct is the conclusion of the following general lemma about maps:

$\left.\llbracket l \subseteq_{m}[V s[\mapsto] l s] ; l^{\prime} \subseteq_{m}\left[V s[\mapsto] l s^{\prime}, V \mapsto v\right] ; V \in \operatorname{set} V s \Longrightarrow l s_{[\text {index } V s \quad V]}=l s_{[\text {index } V s}^{\prime} V\right] ;$

$|l s|=\left|l s^{\prime}\right| ;|V s|<\left|l s^{\prime}\right| \rrbracket$

$\Longrightarrow l^{\prime}(V:=l V) \subseteq_{m}\left[V s[\mapsto] l s^{\prime}\right]$.

It is proved via the chain $\left.l^{\prime}(V:=l V) \subseteq_{m}\left[V s[\mapsto] l s^{\prime}, V \mapsto v\right](V:=l V)\right)=[V s$ $\left.[\mapsto] l s^{\prime}\right](V:=l V) \subseteq_{m}\left[V s[\mapsto] l s^{\prime}\right]$, where the rightmost $\subseteq_{m}$ is proved by a case distinction on whether $l V$ is None or not.

The first premise of the lemma is one of our assumptions. The second premise (with $v$ being $l s_{[|V s|]}^{\prime}$ ) follows from (2) because $|V s|<|l s|$, which follows from the assumption $|V s|+$ max-vars $e \leq|l s|$. Premise $|l s|=\left|l s^{\prime}\right|$ holds because $J_{1}$ evaluation does not change the length of the list of local variables. Thus, we are left with the premise

$V \in$ set $\left.\left.V s \Longrightarrow l s_{[\text {index } V s} V\right]=l s_{[\text {index } V s}^{\prime} V\right]$

which follows because evaluation does not modify hidden local variables. We call an element in a list hidden if it occurrs again further to the right:

hidden :: 'a list $\Rightarrow$ nat $\Rightarrow$ bool

hidden $x s i \equiv i<|x s| \wedge x s_{[i]} \in \operatorname{set}(\operatorname{drop}(i+1) x s)$.

Now we introduce a predicate unmod :: $\operatorname{expr}_{1} \Rightarrow$ nat $\Rightarrow$ bool (Figure 36) such that unmod $e i$ means $i$ is not assigned to in $e$. It is easy to prove that hidden variables are unmodified and that evaluation preserves unmodified variables:

hidden Vs $i \Longrightarrow \operatorname{unmod}\left(\operatorname{comp}_{1}\right.$ Vse) $i$

$\llbracket P \vdash_{1}\langle e,(h, l s)\rangle \Rightarrow\left\langle e^{\prime},\left(h^{\prime}, l s^{\prime}\right)\right\rangle ;$ unmod e $i ; i<|l s| \rrbracket \Longrightarrow l s_{[i]}=l s_{[i]}^{\prime}$.

Now assume $V \in$ set $V s$. It follows easily that hidden $V s_{0}$ (index $V s V$ ), hence that unmod ( $\left.\operatorname{comp} E_{1} V s_{0} e\right)($ index $V s V)$, and hence that $\left.\left.l s_{[\text {index } V s} V\right]=l s_{[\text {index } V s}^{\prime} V\right]$, thus proving the remaining premise. 


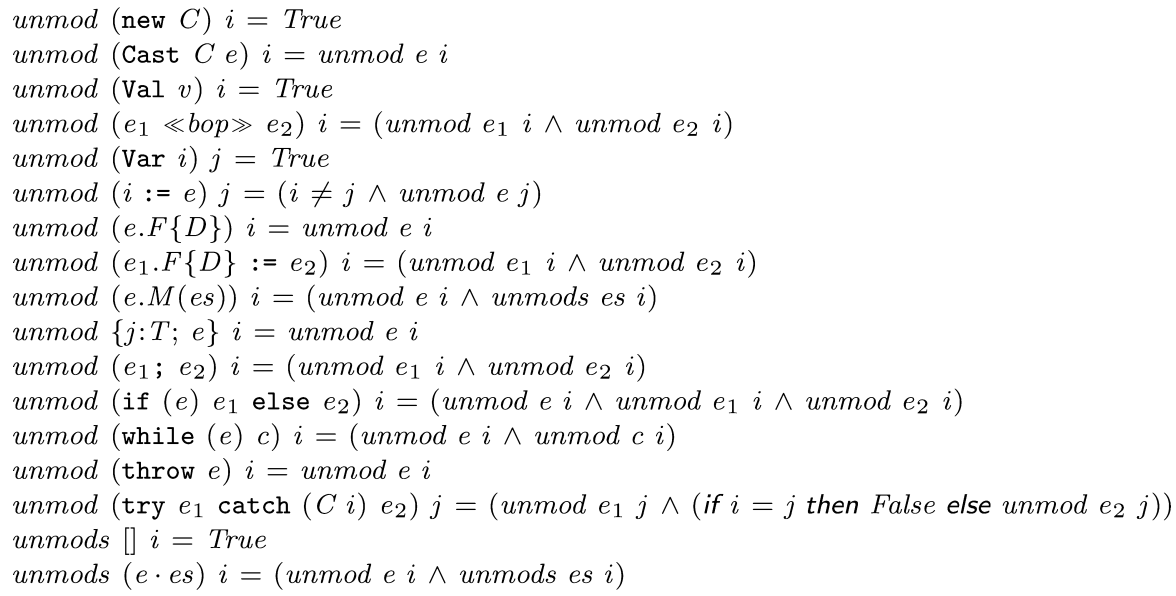

Fig. 36. Definition of unmod .

\subsection{Correctness of Stage 2}

We need to prove that evaluation on the $J_{1}$-level implies a related execution sequence on the JVM-level:

Theorem 5.2. If $P_{1} \vdash C$ sees $M: T s \rightarrow T=$ body in $C$ and $P_{1} \vdash_{1}\langle$ body, $(h, l s)\rangle$ $\Rightarrow\left\langle e^{\prime},\left(h^{\prime}, l s^{\prime}\right)\right\rangle$ then comp $P_{2} P_{1} \vdash($ None $, h,[([], l s, C, M, 0)]) \stackrel{\text { jvm }}{\longrightarrow}\left(\right.$ exception $e^{\prime}$, $\left.h^{\prime},[]\right)$.

Essentially, this theorem says that when executing a method body we obtain the same final heap on the JVM-level as on the $J_{1}$-level. The local variables have disappeared from the final JVM state because we are right at the end of the computation, where the frame stack is empty. Function exception of type ' $a$ exp $\Rightarrow$ addr option extracts the exception reference if there is one: exception $($ Throw $a)=\lfloor a\rfloor$ and exception ${ }_{-}=$None.

Unfortunately, this theorem needs to be strengthened greatly before it becomes provable by induction. The result is

Lemma 5.3. Let $P_{1}:: J_{1}-p r o g, e::$ expr $_{1}$ and $P \equiv \operatorname{comp} P_{2} P_{1}$. Assume $P, C, M, p c \triangleright c o m p E_{2} e$ and $P, C, M \triangleright c_{0} \operatorname{comp}_{2}$ e pc $|v s| / I,|v s|$ and $\left\{p c . .<p c+\left|c o m p E_{2} e\right|\right\} \subseteq I$. If $P_{1} \vdash_{1}\langle e,(h, l s)\rangle \Rightarrow\left\langle e^{\prime},\left(h^{\prime}, l s\right)\right\rangle$, then, letting $\sigma \equiv$ (None, $h,(v s, l s, C, M, p c) \cdot f s)$, there are two cases:

-If $e^{\prime}=\mathrm{Val} v$ then

$P \vdash \sigma \stackrel{\mathrm{jvm}}{\longrightarrow}\left(\right.$ None, $\left.h^{\prime},\left(v \cdot v s, l s^{\prime}, C, M, p c+\left|c o m p E_{2} e\right|\right) \cdot f s\right)$.

-If $e^{\prime}=$ Throw a then there is a pc'such that $p c \leq p c^{\prime}<p c+\left|c o m p E_{2} e\right|$ and

$\neg$ caught $P p c^{\prime} h^{\prime} a$ (compxE $E_{2}$ e pc|vs|) and

$P \vdash \sigma \stackrel{\mathrm{jvm}}{\longrightarrow}$ find-handler $P$ a $h^{\prime}\left(\left(v s, l s^{\prime}, C, M, p c^{\prime}\right) \cdot \mathrm{fs}\right)$.

We will discuss the various components of this lemma in turn.

Instead of a method body, we allow the arbitrary expression $e$. As a consequence, we require that the $p c$ in the top frame points to the compiled expression. To this end we define 
$P, C, M, p c \triangleright i s \equiv i s \leq d r o p$ pc (instrs-of $P C M$ )

$P, C, M, p c(i \equiv \exists i s$. drop pc (instrs-of $P C M)=i \cdot i s$,

where $P, C, M, p c \triangleright i s$ means that the instruction list is is a prefix $(\leq)$ of the instructions of method $M$ starting at $p c$, and $P, C, M, p c$ ( $i$ means that $i$ is the instruction at $p c$ in $M$. Exception handling complicates matters further. We need to say that the exception table compiled from $e$ is contained in the exception table of $M$ in such a way that exceptions thrown by $e$ are not erroneously caught by exception table entries further to the left (because exception tables are searched from the left). We also need to say that if an exception thrown by $e$ is caught by an entry further to the right, that entry does not expect more elements on the stack than we currently have. This is what $P, C, M \triangleright c_{m p x}$ e pc $|v s| / I,|v s|$ expresses:

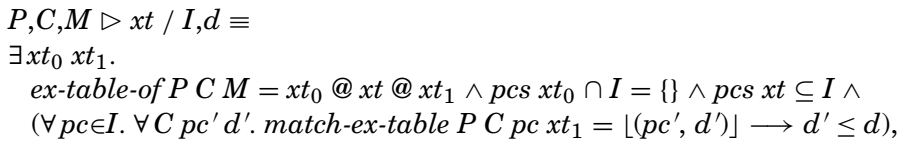

where ex-table-of PC $M$ returns the exception table component of method $P C M$, and $p c s:: e x$-table $\Rightarrow p c$ set yields all program counters guarded by some entry in the table: $p c s x t \equiv \bigcup_{(f, t, C, h, d) \in \text { set } x t}\{f . .<t\}$. The requirement concerning the exception entries to the right of $x t$ is expressed via function match-ex-table, which we describe informally: match-ex-table $P C p c x t_{1}$ searches $x t_{1}$ for an entry matching an exception of class $C$ thrown at $p c$. If it finds a suitable entry, it returns the corresponding pair $\left(p c^{\prime}, d^{\prime}\right)$ of the handler pc and the size the stack has to be reduced to, which must be less than or equal to $d$.

Let us now turn to the conclusion of the lemma. If the $J_{1}$ evaluation ends in a value $v$, there is a corresponding JVM execution which deposits $v$ on top of the operand stack. If the $J_{1}$ evaluation ends with an exception, there must be a $p c^{\prime}$ where the corresponding JVM exception is raised. This $p c^{\prime}$ must lie within the instruction list generated from $e$, the exception (identified by $p c^{\prime}$ and the class of the exception object $h^{\prime} a$ ) must not be caught by an exception handler in $e$, and the final JVM state is the one reached by searching the frame stack for a matching handler. The definition of caught is straightforward and omitted.

Proof. Lemma 5.3 is proved by induction on $P_{1} \vdash_{1}\langle e,(h, l s)\rangle \Rightarrow\left\langle e^{\prime},\left(h^{\prime}, l s^{\prime}\right)\right\rangle$. Each case is a lengthy combination of JVM executions of the compiled subexpressions of $e$ (obtained from the induction hypotheses) to obtain the JVM execution of $c o m p E_{2} e$. We will sketch one case, the penultimate rule in Figure 4, which describes the evaluation of $e=\operatorname{try} e_{1} \operatorname{catch}\left(C^{\prime} V\right) e_{2}$ when $e_{1}$ throws an exception of a subclass of $C^{\prime}$. Let $\sigma_{0}=\left(\right.$ None, $\left.h_{0},\left(v s, l s_{0}, C, M, p c\right) \cdot f s\right), p c_{1}=$ $p c+\left|c o m p E_{2} e_{1}\right|$, and $\sigma_{0}^{\prime}=\left(\right.$ None, $h_{1},\left(\right.$ Addra $\left.\left.\cdot v s, l s_{1}, C, M, p c_{1}+1\right) \cdot f s\right)$.

First, we show $P \vdash \sigma_{0} \stackrel{\text { jvm }}{\longrightarrow} \sigma_{0}^{\prime}$. From the induction hypothesis for the evaluation of $e_{1}$ we obtain $p c^{\prime}$ such that $p c \leq p c^{\prime}<p c_{1}$ and $\neg$ caught $P p c^{\prime} h_{1} a$ (compx $\left.E_{2} e_{1} p c|v s|\right)$ and $P \vdash \sigma_{0} \stackrel{\text { jvm }}{\longrightarrow}$ find-handler $P a h_{1}\left(\left(v s, l s_{1}, C, M, p c^{\prime}\right) \cdot f s\right)$. It remains to show that find-handler returns $\sigma_{0}{ }^{\prime}$, which is the case if it reaches the entry at the end of $x t=c o m p x E_{2}$ e pc $|v s|=c o m p x E_{2} e_{1} p c|v s| @ c o m p x E_{2}$ $e_{2}\left(p c_{1}+2\right)|v s| @\left[\left(p c, p c_{1}, C, p c_{1}+1,|v s|\right)\right.$. From the assumption $P, C, M \triangleright x t$ 
$/ I,|v s|$, it follows that ex-table-of $P C M$ is of the form $x t_{0} @ x t @ x t_{1}$ and that pcs $x t_{0}$ and $I$ are disjoint. Because $p c \leq p c^{\prime}<p c_{1}$ implies $p c^{\prime} \in\{p c . .<p c+$ $\left.\left|c o m p E_{2} e\right|\right\}$ and because by assumption $\left\{p c . .<p c+\left|c o m p E_{2} e\right|\right\} \subseteq I$, we cannot have $p c^{\prime} \in p c s x t_{0}$, that is, the exception is not protected by an entry in $x t_{0}$. We already know that it is not caught by compx $E_{2} e_{1} p c|v s|$ and it cannot be caught by compx $E_{2} e_{2}\left(p c_{1}+2\right)|v s|$ either because all of its entries protect program counters $\geq p c_{1}+2>p c^{\prime}$. Thus, find-handler reaches the matching entry at the end of $x t$.

Executing the Store instruction yields $P \vdash \sigma_{0}{ }^{\prime} \stackrel{\mathrm{jvm}}{\longrightarrow} \sigma_{1}$, where $\sigma_{1}=$ (None, $h_{1}$, (vs, $\left.\left.l s_{1}[i:=A d d r a], C, M, p c_{1}+2\right) \cdot f s\right)$. Let $p c_{2}=p c_{1}+2+\left|c o m p E_{2} e_{2}\right|$. If the evaluation of $e_{2}$ ends in Val $v$, it follows easily from the second induction hypothesis that $P \vdash \sigma_{1} \stackrel{\mathrm{jvm}}{\longrightarrow}\left(\right.$ None, $\left.h_{2},\left(v \cdot v s, l s_{2}, C, M, p c_{2}\right) \cdot f r s\right)$, as required. If it ends in an exception Throw $x a$, the second induction hypothesis yields a $p c^{\prime \prime}$ such that $p c_{1}+2 \leq p c^{\prime \prime}<p c_{2}$ and $\neg$ caught $P p c^{\prime \prime} h_{2} x a\left(\operatorname{compxE}_{2} e_{2}\left(p c_{1}+2\right)\right.$ $|v s|)$ and $P \vdash \sigma_{1} \stackrel{\mathrm{jvm}}{\longrightarrow} \sigma_{2}$, where $\sigma_{2}=$ find-handler $P$ xa $h_{2}\left(\left(v s, l s_{2}, C, M, p c^{\prime \prime}\right) \cdot f s\right)$. This $p c^{\prime \prime}$ will also be the witness of the overall goal. We need to show that $p c$ $\leq p c^{\prime \prime}<p c+\left|c o m p E_{2} e\right|$, which is trivial, that the exception is not caught by $x t$, which follows because it is not caught by compx $E_{2} e_{2}\left(p c_{1}+2\right)|v s|$ and the remaining entries of $x t$ all protect program counters $<p c_{1}<p c^{\prime \prime}$, and that $P$ $\vdash \sigma_{1} \stackrel{\text { jvm }}{\longrightarrow} \sigma_{2}$, which we already have.

The proof of Theorem 5.2 from Lemma 5.3 is straightforward.

\subsection{Main Correctness Theorem}

Composing Theorems 5.1 and 5.2 yields the main correctness theorem.

Theorem 5.4. If wwf-J-prog $P$ and $P \vdash C$ sees $M: T s \rightarrow T=(p n s, b o d y)$ in $C$ and $P \vdash\langle$ body, $(h,[$ this $\cdot$ pns $[\mapsto] v s])\rangle \Rightarrow\left\langle e^{\prime},\left(h^{\prime}, l^{\prime}\right)\right\rangle$ and $|v s|=\mid$ pns $\mid+1$ and $\mid$ rest $\mid=$ max-vars body, then J2JVM $P \vdash($ None, $h,[([]$, vs @ rest, $C, M, 0)])$ $\stackrel{\mathrm{jvm}}{\longrightarrow}\left(\right.$ exception $\left.e^{\prime}, h^{\prime},[]\right)$.

If the program is weakly well-formed, then a big step evaluation of a Jinja method body implies a corresponding JVM execution of the compiled program. The initial JVM state contains the same heap, an empty operand stack, and a list of local variables consisting of the parameter values $v s$ followed by an arbitrary rest which only needs to be of the right length max-vars body.

We may also want to prove the converse: Any result produced by the compiled program can be produced by the source program. Given the preceeding correctness result and the fact that the JVM is deterministic, it would suffice to prove that if the compiled program terminates, so does the source program. We have not done so.

\subsection{Preservation of Well-formedness: Stage 1}

Now we turn from the semantics to the question of type correctness and general well-formedness of the generated code. Preservation of well-typedness by $c o m p E_{1}$ is easily proved by induction on $e$ : 
Lemma 5.5. If $P,[V s[\mapsto] T s] \vdash e:: U$ and $|T s|=|V s|$, then compP f $P, T s \vdash_{1}$ compE $_{1}$ Vs e :: U.

Because program compilation compP does not modify the types, which is reflected in a number of lemmas such as compP $f P \vdash T \leq T^{\prime}$ iff $P \vdash T \leq T^{\prime}$, it does not matter what $f$ is.

The preservation of definite assignment requires a sequence of lemmas, all proved by induction on $e$, the first three automatically.

If $\mathcal{A} e=\lfloor A\rfloor$ then $A \subseteq f v e$

If $\mathcal{A} e=$ None then $\mathcal{A}\left(\operatorname{comp} E_{1}\right.$ Vs e $)=$ None

D e None

If $\mathcal{A} e=\lfloor A\rfloor$ and fve $e \subseteq$ set $V s$ then $\mathcal{A}\left(\operatorname{comp} E_{1} V s e\right)=\lfloor$ index $V s$ ' $A\rfloor$.

If $A \subseteq$ set $V s$ and $f v e \subseteq$ set $V s$ and $\mathcal{D} e\lfloor A\rfloor$ then $\mathcal{D}\left(\operatorname{comp}_{1} V s e\right)\lfloor$ index $V s$ ' $A\rfloor$.

Here $f^{\prime} A$ is the image of $A$ under $f: f^{\prime} A \equiv\{y \mid \exists x \in A . y=f x\}$. The final lemma has the following corollary:

Corollary 5.6 If $\mathcal{D} e\lfloor$ set $V s\rfloor$ and $f v e \subseteq$ set $V s$ and distinct $V s$, then $\mathcal{D}\left(\operatorname{compE}_{1} V s e\right)\lfloor\{. .<|V s|\}\rfloor$.

The combination of this corollary (applicable because all parameter names of a well-formed method are distinct and do not contain this), Lemma 5.5, and the easy lemma $\mathcal{B}\left(\right.$ comp $_{1} V s$ e) $|V s|$ yields

THEOREm 5.7. If $w f-J-p r o g ~ P$, then $w f-J_{1}-\operatorname{prog}\left(\operatorname{comp} P_{1} P\right)$.

The proof is straightforward using the lemma (where $\bigwedge$ is universal quantification)

$\llbracket \wedge C M T s T m$.

$\llbracket P \vdash C$ sees $M: T s \rightarrow T=m$ in $C ; w f-m d e c l w f_{1} P C(M, T s, T, m) \rrbracket$

$\Longrightarrow w f-m d e c l w f_{2}(\operatorname{compP} f P) C(M, T s, T, f m)$;

$w f$-prog $w f_{1} P \rrbracket$

$\Longrightarrow w f-p r o g w f_{2}(\operatorname{compP} f P)$,

which essentially says that compPf turns a $w f_{1}$ well-formed program into a $w f_{2}$ well-formed program if $f$ turns a $w f_{1}$ well-formed method into a $w f_{2}$ well-formed method.

\subsection{Preservation of Well-formedness: Stage 2}

We have to show that $\operatorname{comp} E_{2}$ maps a well-typed $J_{1}$ expression into a welltyped instruction list. For that purpose, we follow Stärk et al. [2001] and define a "type compiler" that compiles an expression into type annotations (a method type) for the instruction list that $\operatorname{comp}_{2}$ produces:

compT :: ty list $\Rightarrow$ nat set option $\Rightarrow$ ty list $\Rightarrow \operatorname{expr}_{1} \Rightarrow t y_{i}{ }^{\prime}$ list. 
As in Section 4.7, we fix the program and the method we are in:
$P \quad:: J_{1}$-prog the program,
$m x l::$ nat number of local variables,
$m x s::$ nat maximum stack size,
$T_{r} \quad:: t y$ and return type of the method.

The type compiler call compT E A ST e produces the method type for comp $E_{2} e$ in the context of the environment $E$ assuming that initially (a) the local variables in $A::$ nat set option are initialised, and (b) the stack elements are typed according to $S T$ :: ty list. It uses the following auxiliary functions:

$t y_{l} E A^{\prime} \equiv \operatorname{map}\left(\lambda i\right.$. if $i \in A^{\prime} \wedge i<|E|$ then $O K E_{[i]}$ else Err $)[0 . .<m x l]$

computes the typing of the local variables based on their declared types in $E$ and their initialisation status in $A^{\prime}::$ nat set.

$t y_{i}{ }^{\prime} S T E A \equiv$ case $A$ of None $\Rightarrow$ None $\left\lfloor\left\lfloor A^{\prime}\right\rfloor \Rightarrow\left\lfloor\left(S T, t y_{l} E A^{\prime}\right)\right\rfloor\right.$

computes the state type described by $S T, E$, and $A$. Remember that $A=$ None indicates unreachability.

after $E A S T e \equiv t y_{i}{ }^{\prime}(t y E e \cdot S T) E(A \sqcup \mathcal{A} e)$

produces the state type characterising stack and local variables after the evaluation of $e$. Thus, we need to push the type of $e$ onto the stack and take the effect of $e$ on $A$ into account. Function $t y$ is a functional version of type checking and is characterised by $P, E \vdash_{1} e:: T \Longrightarrow t y E e=T$

The full definition of compT is shown in Figure 37 and uses the additional function

$\operatorname{compT}_{a} E A S T e \equiv \operatorname{compTEASTe} @[$ after E A ST e]

because the method type that compT produces (intentionally) lacks the state types directly before and after the execution of the corresponding instruction list. That is, it is one element shorter than the instruction list, describing only the states between the instructions. As a simple example, let $e$ be the expression $1:=$ Val (Intg 42) and hence,

$\operatorname{compE}_{2} e=[$ Push (Intg 42), Store 1, Push Unit].

With $E=[$ Class $C$, Integer $], A=\lfloor\{0\}\rfloor$ and $S T=[]$ we obtain

compT E A ST e = [ᄂ([Integer $],[O K($ Class C), Err $])\rfloor, ~[([],[O K($ Class C), OK Integer $])]]$.

Since we are dealing with instruction lists in isolation rather than with whole methods, we need a new well-typedness notion. We write $\vdash i s, x t[::] \tau s$ to mean that the instruction list is together with the exception table $x t$ is well-typed w.r.t. the method type $\tau s:: t y_{i}{ }^{\prime}$ list:

$\vdash i s, x t[::] \tau s \equiv$

$|i s|<|\tau s| \wedge p c s x t \subseteq\{0 . .<|i s|\} \wedge\left(\forall p c<|i s| . P, T_{r}, m x s,|\tau s|, x t \vdash i s_{[p c]}, p c:: \tau s\right)$.

The key theorem says that the instruction list and exception table are welltyped w.r.t. the method type produced by the type compiler:

ACM Transactions on Programming Languages and Systems, Vol. 28, No. 4, July 2006. 


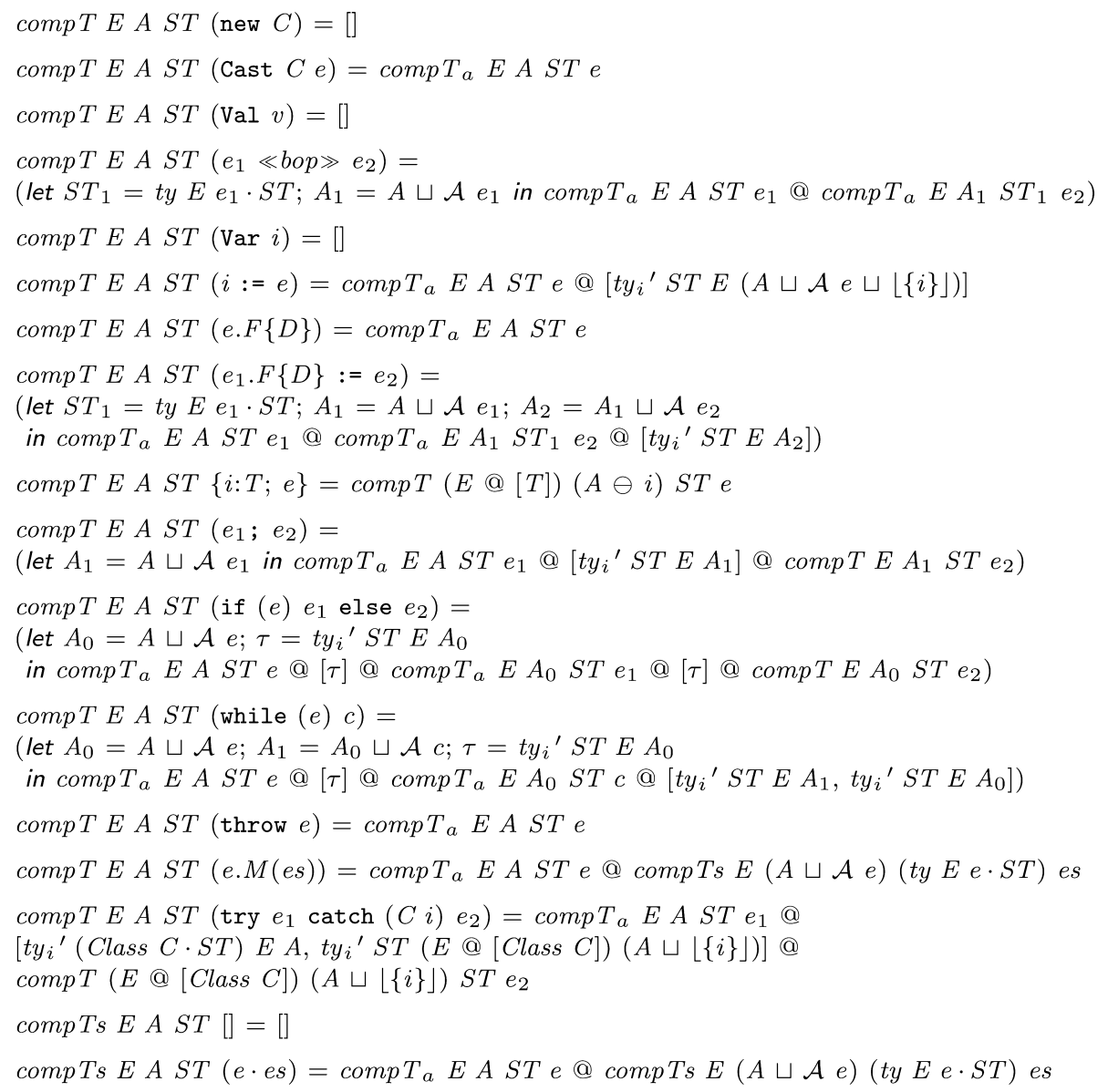

Fig. 37. Type compiler.

Theorem 5.8. If $P, E \vdash_{1} e:: T$ and $\mathcal{D} e A$ and $\mathcal{B} e|E|$ and $|S T|+$ max-stack $e \leq$ mxs and $|E|+$ max-vars $e \leq m x l$, then $\vdash \operatorname{comp}_{2}$ e, compxE $E_{2}$ e $0|S T|[::] t y_{i}{ }^{\prime} S T E A \cdot \operatorname{comp} T_{a} E$ A ST e.

PRoof. By induction on $e$. In order to combine different well-typedness propositions we need a central lemma which follows readily from the definitions:

LEMMA 5.9. If $\vdash i s_{1}, x t_{1}[::] \tau s_{1} @ \tau s_{2}$ and $\vdash i s_{2}, x t_{2}[::] \tau s_{3}$ and $\left|\tau s_{1}\right|=\left|i s_{1}\right|$ and $\tau s_{3} \leq \tau s_{2}$ then $\vdash i s_{1} @ i s_{2}, x t_{1} @$ shift $\left|i s_{1}\right| x t_{2}[::] \tau s_{1} @ \tau s_{2}$.

Here shift $n x t$ shifts all program counters in $x t$ by $n$ and $\leq$ again means prefix. This lemma works well in the presence of forward jumps, but for backward ones we need another:

Lemma 5.10. If $\vdash i s, x t[::]$ $\tau$ and $P, T_{r}, m x s, m p c,[] \vdash i, p c:: \tau s$ and $p c=|i s|$ and $m p c=|\tau s|$ and $|i s|+1<|\tau s|$, then $\vdash i s @[i], x t[::] \tau s$. 


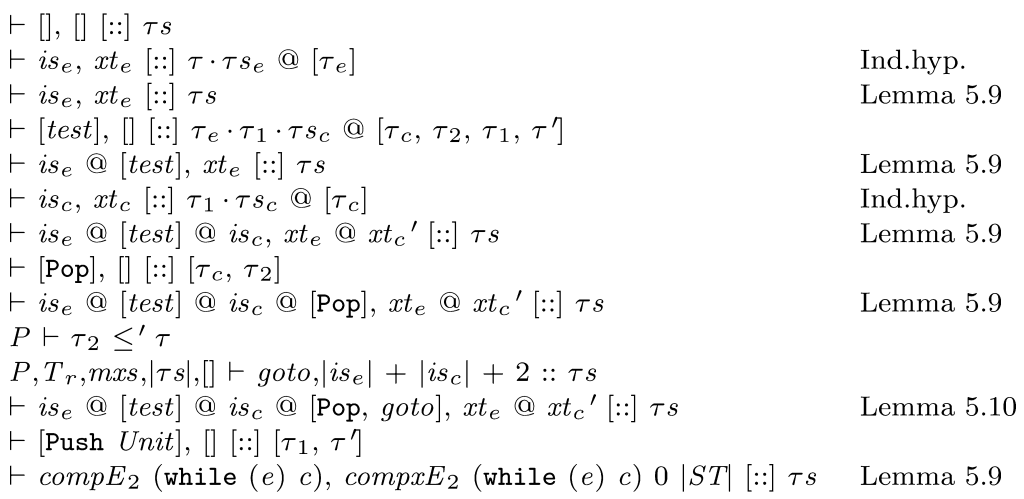

Fig. 38. Proof summary for while.

We present just one case of the proof of Theorem 5.8, the while-loop. The correspondence of instructions $c o m p E_{2}$ (while $\left.(e) c\right)$ (first column) and types compTEAST (while $(e) c$ ) (second column) is best described by a table:

$\begin{array}{lll}\text { compE } E_{2} e & \tau \cdot \tau s_{e} & \text { where } \tau=t y_{i}{ }^{\prime} S T E A \text { and } \tau s_{e}=\text { compTEASTe } \\ \text { IfFalse } \ldots & \tau_{e} & \text { where } \tau_{e}=t y_{i}{ }^{\prime}(\text { Boolean } S T) E A_{0} \text { and } A_{0}=A \sqcup \mathcal{A} e \\ \text { compE } E_{2} c & \tau_{1} \cdot \tau s_{c} & \text { where } \tau_{1}=t y_{i}{ }^{\prime} S T E A_{0} \text { and } \tau s_{c}=c o m p T E A_{0} S T c \\ \text { Pop } & \tau_{c} & \text { where } \tau_{c}=t y_{i}{ }^{\prime}(t y E c \cdot S T) E A_{1} \text { and } A_{1}=A_{0} \sqcup \mathcal{A} c \\ \text { Goto } \ldots & \tau_{2} & \text { where } \tau_{2}=t y_{i}{ }^{\prime} S T E A_{1} \\ \text { Push Unit } & \tau_{1} & \\ & \tau^{\prime} & \text { where } \tau^{\prime}=t y_{i}{ }^{\prime}(\text { Void } \cdot S T) E A_{0}\end{array}$

Using the abbreviations $i s_{e}=\operatorname{comp}_{2} e, x t_{e}=\operatorname{compxE}_{2} e 0|S T|, i s_{c}=\operatorname{compE}_{2} c$, $x t_{c}=$ comp $x E_{2} c 0|S T|, x t_{c}{ }^{\prime}=$ shift $\left(\left|i s_{e}\right|+1\right) x t_{c}$, test $=$ IfFalse $\ldots$, goto $=$ Goto $\ldots$ and $\tau s=\tau \cdot \tau s_{e} @\left[\tau_{e}, \tau_{1}\right] @ \tau s_{c} @\left[\tau_{c}, \tau_{2}, \tau_{1}, \tau\right]$ (i.e., $\tau s=\operatorname{compT} E A S T$ (while $(e) c)$ ), we can summarize the proof in the table in Figure 38 where the uncommented lines follow easily.

The remaining cases follow the same pattern. Try-catch requires

Lemma 5.11. If $\left|i s_{1}\right|=\left|\tau s_{1}\right|$ and is-class $P C$ and $|S T|<m x s$ and $\vdash i s_{1} @ i s_{2}, x t[::]$ $\tau \mathrm{s}_{1} @\left(\operatorname{ty}_{i}{ }^{\prime}\left(\right.\right.$ Class C.ST) E A. $\left.\tau \mathrm{s}_{2}\right)$ and

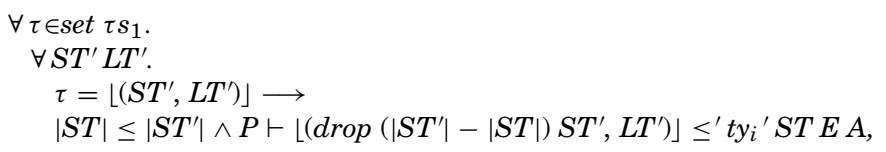

then $\vdash i s_{1} @ i s_{2}, x t @\left[\left(0,\left|i s_{1}\right|-1, C,\left|i s_{1}\right|,|S T|\right)\right][::] \tau s_{1} @\left(t y_{i}{ }^{\prime}(\right.$ Class $\left.C \cdot S T) E A \cdot \tau s_{2}\right)$.

to justify the additional exception table entry.

Now it is not very difficult to conclude the next theorem.

Theorem 5.12. If $w f-J_{1}$-prog $P$, then $w f$-jum-prog $\left(\operatorname{comp} P_{2} P\right)$.

By definition of $w f$-jum-prog we need a table $\Phi$ such that $w f$-jum-prog ${ }_{\Phi}\left(\operatorname{compP}_{2}\right.$ P). The table can be computed by compTP :: $J_{1}-$ prog $\Rightarrow t y_{P}$

ACM Transactions on Programming Languages and Systems, Vol. 28, No. 4, July 2006. 
compTP P C $M \equiv$

let $(D, T s, T, e)=$ method $P C M ; E=$ Class $C \cdot T s ; A=\lfloor\{. .|T s|\}\rfloor$;

$m x l=1+|T s|+$ max-vars $e$

in $t y_{i}{ }^{\prime} m x l[] E A \cdot \operatorname{comp} T_{a} P \operatorname{Pxl} E A[] e$

and the proof of $w f-j v m-\operatorname{prog}_{\Phi}$ follows largely from Theorem 5.8. Note that $t y_{i}{ }^{\prime}$ and $\operatorname{comp} T_{a}$ are explicitly supplied with their implicit arguments $m x l$ and $P$ because these are no longer fixed globally but are the specific values in this definition.

\subsection{Preservation of Well-Formedness: Main Theorem}

Combining Theorems 5.7 and 5.12 yields the main theorem:

Theorem 5.13. If wf-J-prog $P$, then wf-jum-prog (J2JVM P).

If the source program is well-formed - which includes every method body being well-typed - then the compiled program is also well-formed and will pass bytecode verification. This is a nontrivial property not guaranteed by Java compilers (for example, JDK 1.2 and 1.3) [Stärk and Schmid 2001].

\subsection{Related Work}

There is a sizable amount of literature on compiler verification in general [Dave 2003] which we cannot possibly survey here, but very little specifically for Java. An early landmark in mechanically verified compilers is the work by Young [1989] whose source language has procedures but no OO features, but whose target language is a real rather than a virtual machine. The work by Strecker [2002] and Klein and Strecker [2004] is closely related to ours, but their main theorem does not consider exceptions, which is a considerable simplification. Furthermore, their type compiler intrinsically assumes that variables are initialized and do not change their type (which they can if you have local variables and reuse storage as we do).

Stärk et al. [2001] show that terminating computations are compiled into terminating ones, just as we have done. They also claim to prove a 1:1 correspondence between Java and JVM executions. However, their main theorem fails to imply that nonterminating computations are compiled into nonterminating ones (which we have not shown, either). Its statement and proof need to be augmented to prove that one cannot have a diverging Java computation in correspondence with a terminating JVM execution. Furthermore, it is interesting to note that their compiler has one stage only. Our stage 1 disappears in the sentence "we suppress the details of a consistent assignment of JVM variable numbers $\bar{x}$ to (occurrences of) Java variables $x$," combined with the fact that their Java semantics treats local variables like the JVM: Upon exit of a block, the local variables of the block are unchanged and still visible. This works only because Java forbids nested declarations of the same variable, which Jinja allows. Finally, it is interesting to note that their formalization uses attributed syntax trees that tell for example, what the current environment $E$ of each subexpression is, whereas we pass $E$ explicitly into many of our functions. Although this is true for the whole formalization, it is most noticeable for the 
type compiler with its lengthy parameter list. It may well reduce clutter in our formalization to work with expressions annotated with $E$, etc. We have not done so to minimize the number of concepts needed.

The correctness of compiling exceptions is studied by Hutton and Wright [2004]. They treat a very simple expression language and a stack machine where all exception handling information is kept on the stack. Because there is no separate exception table, this leads to simpler exception handling on the machine level and simpler proofs. It is possible that their machine could serve as a convenient stepping stone between Jinja and the Jinja VM.

League et al. [2002] compile Featherweight Java into $F_{\omega}$, which is very different from our compilation into the JVM.

\section{CONCLUSION}

We have given a completely formal account of the core of a Java-like sequential language, abstract machine, and compiler. Although there are some interesting contributions to the analysis of individual facets, for example, definite assignment, the emphasis is on a unified model. At the same time, we have demonstrated that it is possible to present this model in a style appropriate for a scientific journal, although the formal and machine-checked metalanguage prohibits some of the liberties (like "...") of traditional journal articles (and we have decided not to take others like hiding injections such as $\lfloor. J$ ). Of course, Jinja is still a small language compared to Java, but we hope that our theories will become the basis for further extensions of Jinja, just as others, for example, Büchi and Weck [1998], have extended some of our earlier Java formalisations.

The whole development (excluding this article) runs to 20000 lines of Isabelle/HOL text (with few comments), roughly 350 printed pages, and just over 1000 theorems (available at www.in.tum.de/ nipkow/Jinja/). The proofs take about 25 minutes to process on a $3 \mathrm{GHz}$ Pentium IV with 1GB RAM. This is a major investment, but one that can be built up to gradually: from type checking via prototyping to full-blown theorem proving. The early stages are cheap and worthwhile for any language definition, the final stage is more of an investment but yields a considerable increase in confidence-particularly since the proofs are structured and the general drift of an argument can be followed even by a non-theorem-proving expert.

We have already hinted that the whole formalisation is executable (with the exception of $w f$-jum-prog, a specification that is implemented by the executable BV). That is, Isabelle/HOL supports the translation of a mixture of recursive functions and inductive relations into executable (ML) code [Berghofer and Nipkow 2002; Berghofer 2003] and we have run test cases to increase our confidence in the intuitive correctness of our definitions. Although we have not broached this aspect at all, we consider prototyping essential for projects such as ours, where the initial specification (here, the source language) is already too large to be "obviously" correct. In addition, we consider these executable ML prototypes as reference implementations for the language, the compiler, the bytecode verifier, etc. Although these prototypes do not have the performance required for production purposes, theorem provers offer the right environment for 
achieving the required performance: Efficient programs can be proved correct w.r.t. initial specifications and then exported to ML just like the prototypes. In fact, the Jinja code generator is a standard recursive functional program without particular performance bottlenecks and may not need much tuning. The bytecode verifier is another matter because its fixed point engine passes a large list around in a single-threaded manner, but the generated ML code does not take advantage of this. ACL2 and PVS have code generators that could safely implement this list by an array that is updated destructively. There is ongoing work to provide similar features in Isabelle. We are convinced that theorem provers provide the right means to develop certified efficient language processors that are largely functional but imperative where necessary. Liu and Moore [2003] already provide strong evidence for this thesis.

\section{ACKNOWLEDGMENTS}

This work is directly based on and would not exist without the Isabelle formalisations of various facets of Java by David von Oheimb, Conny Pusch, Norbert Schirmer, Martin Strecker and Martin Wildmoser, for which we are very grateful. We also thank them and the referees for commenting on draft versions of this article.

\section{REFERENCES}

Alves-Foss, J., ED. 1999. Formal Syntax and Semantics of Java. LNCS, vol. 1523. Springer, New York.

Ancona, D., Lagorio, G., And Zucca, E. 2001. A core calculus for Java exceptions. In Proceedings of the 16th ACM Conference on Object Oriented Programming, Systems, Languages, and Applications. 16-30.

Aspinall, D. 2000. Proof general-A generic tool for proof development. In Proceedings of the Tools and Algorithms for Construction and Analysis of Systems Conference, TACAS 2000, S. Graf and M. Schwartzbach, eds. LNCS, vol. 1785. Springer, New York, 38-42.

BALLARIN, C. 2003. Locales and locale expressions in Isabelle/Isar. In Proceedings of the Types for Proofs and Programs Conference, TYPES 2003, S. Berardi, et al. eds. LNCS, vol. 3085. Springer, New York, 34-50.

Barthe, G. AND Dufay, G. 2004. A tool-assisted framework for certified bytecode verification. In Proceedings of the Conference Fundamental Approaches to Software Engineering, FASE 2004, M. Wermelinger and T. Margaria, eds. LNCS, vol. 2984. Springer, New York, 99-113.

Barthe, G., Dufay, G., Jakubiec, L., Serpette, B., And de Sousa, S. M. 2001. A formal executable semantics of the JavaCard platform. In Proceedings of the Programming Languages and Systems (ESOP 2001) Conference, D. Sands, ed. LNCS, vol. 2028. Springer, New York, 302319.

Berghofer, S. 2003. Proofs, programs and executable specifications in higher order logic. Ph.D. thesis, Institut für Informatik, Technische Universität München.

Berghofer, S. ANd NiPKow, T. 2002. Executing higher order logic. In Proceedings of the Types for Proofs and Programs (TYPES'00) Conference, P. Callaghan, et al. eds. LNCS, vol. 2277. Springer, New York, 24-40.

Bertelsen, P. 1997. Semantics of Java bytecode. Tech. rep., Technical University of Denmark. Mar. http://home.tiscali.dk/petermb/.

BüCHI, M. AND WeCK, W. 1998. Compound types for Java. In Proceedings of the 13th ACM Conference Object-Oriented Programming, Systems, Languages, and Application. ACM Press, New York,.

Coglio, A. 2004. Simple verification technique for complex Java bytecode subroutines. Concurrency and Comput. Practice and Experience 16, 7 (June), 647-670. 
Coglio, A., Goldberg, A., And QIan, Z. 2000. Toward a provably-correct implementation of the JVM bytecode verifier. In Proceedings of the DARPA Information Survivability Conference and Exposition (DISCEX'00), vol. 2. IEEE Computer Society Press, Las Alamitos, Calif., 403-410.

Cohen, R. 1997. The defensive Java virtual machine specification. Tech. rep., Computational Logic Inc. http://www.cli.com/software/djvm/.

Dave, M. A. 2003. Compiler verification: A bibliography. SIGSOFT Softw. Eng. Notes 28, 6, $2-2$.

Drossopoulou, S. and Eisenbach, S. 1999. Describing the semantics of Java and proving type soundness. In Formal Syntax and semantics of a Java. LNCS, vol.1523. Springer, New York $41-82$.

Flatt, M., Krishnamurthi, S., and Felleisen, M. 1999. A programmer's reduction semantics for classes and mixins. In Formal Syntax and semantics of a Java. LNCS, vol.1523. Springer, New York, 241-269.

Freund, S. N. 2000. Type systems for object-oriented intermediate languages. Ph.D. thesis, Stanford University.

Freund, S. N. And Mitchell, J. C. 2003. A type system for the Java bytecode language and verifier. J. Automated Reasoning 30, 271-321.

GoldBerg, A. 1998. A specification of Java loading and bytecode verification. In Proceedings of the 5th ACM Conference on Computer and Communications Security. ACM Press, New York, 49-58.

Hartel, P. and Moreau, L. 2001. Formalizing the safety of Java, the Java virtual machine and Java card. ACM Comput. Surv. 33, 517-558.

Huisman, M. 2001. Reasoning about Java programs in higher order logic with PVS and Isabelle. Ph.D. thesis, Universiteit Nijmegen.

Hutton, G. AND Wright, J. 2004. Compiling exceptions correctly. In Mathematics of Program Construction, MPC 2004, D. Kozen and C. Shankland, eds. LNCS, vol. 3125. Springer, New York, 211-227.

IgArashi, A., Pierce, B. C., ANd Wadler, P. 2001. Featherweight Java: A minimal core calculus for Java and GJ. ACM Trans. Program. Lang. Syst. 23, 396-450.

KILDALL, G. A. 1973. A unified approach to global program optimization. In Proceedings of the ACM Symposium on Principles of Programming Languages. ACM Press, New York, 194-206.

KLeIN, G. 2003. Verified Java bytecode verification. Ph.D. thesis, Institut für Informatik, Technische Universität München.

KLEIN, G. AND NiPKow, T. 2001. Verified lightweight bytecode verification. Concurrency and Comput. Practice and Experience 13, 1133-1151.

KLeIN, G. AND NiPKow, T. 2003. Verified bytecode verifiers. Theor. Comput. Sci. 298, 583-626.

KLeIN, G. AND StRECKer, M. 2004. Verified bytecode verification and type-certifying compilation. J. Logic Algebraic Program. 58, 1-2, 27-60.

KLein, G. ANd Wildmoser, M. 2003. Verified bytecode subroutines. J. Automated Reasoning 30, 363-398.

League, C., Shao, Z., And Trifonov, V. 2002. Type-Preserving compilation of featherweight Java. ACM Trans. Program. Lang. Syst. 24, 112-152.

Leroy, X. 2003. Java bytecode verification: Algorithms and formalizations. J. Automated Reasoning 30, 235-269.

Lindholm, T. And Yeldin, F. 1999. The Java Virtual Machine Specification. Addison-Wesley, Reading, Mass.

Liv, H. AND Moore, J. S. 2003. Executable JVM model for analytical reasoning: A study. In Proceedings of the 2003 Workshop on Interpreters, Virtual Machines and Emulators. ACM Press, New York 15-23.

Muchnick, S. S. 1997. Advanced Compiler Design and Implementation. Morgan Kaufmann San Fransisco, Calif.

NiPKow, T. 1991. Higher-order critical pairs. In Proceedings of the 6th IEEE Symposium on Logic in Computer Science. IEEE Computer Society Press, Los Alamitos, Calif., 342-349.

Nipkow, T. 2001. Verified bytecode verifiers. In Proceedings of the Foundations of Software Science and Computation Structures Conference, (FOSSACS 2001), F. Honsell, ed. LNCS, vol. 2030. Springer, New York, 347-363.

Nipkow, T., ed. 2003a. Special Issue on Java Bytecode Verification. J. Automated Reasoning, 30, 3-4.

ACM Transactions on Programming Languages and Systems, Vol. 28, No. 4, July 2006. 
Nipkow, T. 2003b. Structured proofs in Isar/HOL. In Proceedings of the Types for Proofs and Programs Conference (TYPES 2002), H. Geuvers and F. Wiedijk, eds. LNCS, vol. 2646. Springer, New York, 259-278.

NiPKow, T. 2005. Jinja: Towards a comprehensive formal semantics for a Java-like language. In Proof Technology and Computation, Proceedings of the Marktobderdorf Summer School Conference 2003, H. Schwichtenberg and K. Spies, eds. IOS Press Amsterdam, the Netherlands.

Nipkow, T. AND OHeimb, D. v. 1998. Java light $_{\text {in }}$ is type-safe-Definitely. In Proceedings of the 25th ACM Symposium on Principles of Programming Languages. ACM Press, New York, 161-170.

Nipkow, T., Paulson, L., ANd Wenzel, M. 2002. Isabelle/HOL-A Proof Assistant for Higher-Order Logic. LNCS, vol. 2283. Springer, New York. http://www. in.tum.de/〜nipkow/LNCS2283/.

Oheimb, D. v. ANd NipKow, T. 1999. Machine-Checking the Java specification: Proving type-safety. In Formal Syntax and semantics of a Java. LNCS, vol.1523. Springer, New York, 119-156.

Pusch, C. 1999. Proving the soundness of a Java bytecode verifier specification in Isabelle/HOL. In Proceedings of the Tools and Algorithms for the Construction and Analysis of Systems Conference (TACAS'99), W. Cleaveland, ed. LNCS, vol. 1579. Springer, New York, 89-103.

QIAN, Z. 2000. Standard fixpoint iteration for Java bytecode verification. ACM Trans. Program. Lang. Syst. 22, 638-672.

Rose, E. 2002. Vérification de code d'octet de la machine virtuelle Java. Formalisation et implantation. Ph.D. thesis, Université Paris VII.

Rose, E. 2003. Lightweight bytecode verification. J. Automated Reasoning 31, 3-4, 303-334.

Rose, E. ANd Rose, K. 1998. Lightweight bytecode verification. In Proceedings of the OOPSLA'98 Workshop on Formal Underpinnings of Java.

Schirmer, N. 2003. Java definite assignment in Isabelle/HOL. In Proceedings of the Formal Techniques for Java-like Programs 2003 Conference, S. Eisenbach et al. eds. Chair of Software Engineering, ETH Zürich. Technical Report 108.

Schirmer, N. 2004. Analysing the Java package/access concepts in Isabelle/HOL. Concurrency and Comput. Practice and Experience 16, 689-706.

STÄRK, R. AND Schmid, J. 2001. The problem of bytecode verification in current implementations of the JVM. Tech. rep., Department of Computer Science, ETH Zürich.

StÄRK, R., Schmid, J., AND Börger, E. 2001. Java and the Java Virtual Machine-Definition, Verification, Validation. Springer, New York.

Stata, R. And Abadi, M. 1998. A type system for Java bytecode subroutines. In Proceedings of the 25th ACM Symposium on Principles of Programming Languages. ACM Press, New York, $149-161$.

Strecker, M. 2002. Formal verification of a Java compiler in Isabelle. In Automated Deduction$C A D E-18$, A. Voronkov, ed. LNCS, vol. 2392. Springer, New York, 63-77.

Syme, D. 1999. Proving Java type soundness. In Formal Syntax and Semantics of Java. LNCS, vol. 1523. Springer, New York. 83-118.

WENZEL, M. 2002. Isabelle/Isar-A versatile environment for human-readable formal proof documents. Ph.D. thesis, Institut für Informatik, Technische Universität München. http://tumb1. biblio.tu-muenchen.de/publ/diss/in/2002/wenzel.html.

Wright, A. And Felleisen, M. 1994. A syntactic approach to type soundness. Inf. Comput. 115, 38-94.

Young, W. D. 1989. A mechanically verified code generator. J. Automated Reasoning 5, 493-518.

Received March 2004; revised January 2005; accepted July 2005 